\title{
Evaluation of a Candidate Classical Biological Control Agent and Critical Assessment of Suggested Host Specificity Testing Guidelines
}

\author{
by \\ Wade Harley Jenner \\ BSc., MSc. \\ A thesis submitted to \\ the Faculty of Graduate Studies and Research \\ in partial fulfillment of \\ the requirements for the degree of
}

Doctor of Philosophy

Department of Biology

Carleton University

Ottawa, Ontario, Canada

October 28, 2008

(C) 2008, Wade Jenner 


$\begin{array}{ll}\begin{array}{l}\text { Library and } \\ \text { Archives Canada }\end{array} & \begin{array}{l}\text { Bibliothèque et } \\ \text { Archives Canada }\end{array} \\ \begin{array}{l}\text { Published Heritage } \\ \text { Branch }\end{array} & \begin{array}{l}\text { Direction du } \\ \text { Patrimoine de l'édition }\end{array} \\ \begin{array}{l}\text { 395 Wellington Street } \\ \text { Ottawa ON K1A 0N4 } \\ \text { Canada }\end{array} & \begin{array}{l}\text { 395, rue Wellington } \\ \text { Ottawa ON K1A 0N4 } \\ \text { Canada }\end{array}\end{array}$

Your file Votre référence ISBN: 978-0-494-47477-8 Our file Notre référence ISBN: 978-0-494-47477-8

NOTICE:

The author has granted a nonexclusive license allowing Library and Archives Canada to reproduce, publish, archive, preserve, conserve, communicate to the public by telecommunication or on the Internet, loan, distribute and sell theses worldwide, for commercial or noncommercial purposes, in microform, paper, electronic and/or any other formats.

The author retains copyright ownership and moral rights in this thesis. Neither the thesis nor substantial extracts from it may be printed or otherwise reproduced without the author's permission.
AVIS:

L'auteur a accordé une licence non exclusive permettant à la Bibliothèque et Archives Canada de reproduire, publier, archiver, sauvegarder, conserver, transmettre au public par télécommunication ou par l'Internet, prêter, distribuer et vendre des thèses partout dans le monde, à des fins commerciales ou autres, sur support microforme, papier, électronique et/ou autres formats.

L'auteur conserve la propriété du droit d'auteur et des droits moraux qui protège cette thèse. $\mathrm{Ni}$ la thèse ni des extraits substantiels de celle-ci ne doivent être imprimés ou autrement reproduits sans son autorisation.
In compliance with the Canadian Privacy Act some supporting forms may have been removed from this thesis.

While these forms may be included in the document page count, their removal does not represent any loss of content from the thesis.
Conformément à la loi canadienne sur la protection de la vie privée, quelques formulaires secondaires ont été enlevés de cette thèse.

Bien que ces formulaires aient inclus dans la pagination, il n'y aura aucun contenu manquant.

\section{Canada}




\section{Abstract}

The leek moth, Acrolepiopsis assectella (Zeller) (Lepidoptera: Acrolepiidae), is a Palaearctic pest of Allium crops. It is considered a threat to crop production and wild Allium biodiversity in Canada, where it was unintentionally introduced and continues to spread. This research explores the potential for a classical biological control programme against leek moth in Canada.

A life table study of leek moth in its native range revealed that total pre-imaginal mortality varied between 96.4 and $99.6 \%$ over the three years of the study. Natural enemies contributed significantly and consistently to mortality in the pupal stage only. The pupal parasitoid, Diadromus pulchellus Wesmael (Hymenoptera: Ichneumonidae) was selected for evaluation as a candidate biological control agent.

Several cold exposure experiments were run to test the cold hardiness of $D$. pulchellus. Parasitoids still developing inside their hosts were less tolerant of extended periods of cool (above-freezing) or freezing temperatures than adults, suggesting that $D$. pulchellus overwinters in the adult stage. Females survived cold treatments better than males and were able to survive for several weeks at temperatures that would be experienced under a layer of snow during the winter months in Canada. Thus, this agent is expected to be suitable for the Canadian climate.

During oviposition trials in the laboratory, motivation to oviposit was influenced by the physiological and informational state of the parasitoids. In particular, parasitoid age and nutritional status both had a significant effect on the number of host pupae killed. In contrast, previous host experience and parasitoid density had weaker and inconsistent effects while mating status, arena size and the presence/absence of the host plant had no observable effect. When young and old $D$. pulchellus females were used in no-choice host specificity tests, there was no difference in the acceptance rate of non-target species. However, changing the ratio of target to non-target hosts in choice tests affected the oviposition response of old, but not young, parasitoids, indicating that there is an age effect. Manipulation of biological control agents to increase their motivation to oviposit in host range tests will require knowledge of the specific effects of endogenous and exogenous factors on their foraging behaviour. 


\section{Acknowledgements}

I owe many thanks to my advisory committee, consisting of Naomi Cappuccino, Peter Mason, Ulli Kuhlmann and John Arnason. Each member showed sincere interest in the research topics from start to finish and was always eager to discuss issues when they arose. This made it an enjoyable and rewarding experience for me.

I thank Eric Thibout for providing a leek moth culture at the start of this project and Erich Diller (Bavarian State Collection of Zoology), Klaus Horstmann (University of Wuerzburg) and Hannes Baur (Natural History Museum of Bern) for identifying leek moth parasitoids. In addition, I am indebted to the Swiss vegetable producers who permitted me to conduct experiments in their leek and onion crops. These include Rolf Etter (Bioleguma, Ried near Kerzers), Roland Fasnacht (Bio Gemüse \& Kräuter, Sugiez), Thomas Keller (Rathgeb's Bioprodukte, Unterstammheim), Stefan Müller (BioLand Agrarprodukte AG, Steinmaur), Dieter Schächtle (Bio-Gemüsebau, Taegerwilen), Toni

Suter (Schwarz Gemuesebau, Villigen), Volker Szepeck (Biotta, Taegerwilen) and Jürg and Erwin Züttel (Gemüse, Müntschemier).

The work in this thesis would not have been possible without the help of the following students and postdoctoral fellows: Lars Andreassen, Favio Corlay, Serge Hämmerli, Tim Haye, Annie Hibbert, Li Hongmei, Emma Hunt, Virginia Larraz, Jenny Lazebnik, Alicia Leroux, Samantha Magnus, Kathy Makela, Jake Miall, C-Jae Morden, Basri Pulaj, Christof Schüpp, Patrick Turko, Mike Wogin, and Amber Zabarauskas. Over the last four and a half years, these people worked alongside me in the field and in the laboratory, sometimes until crazy hours, to get the job done. I would also like to thank Margaret Appleby for keeping me connected with the leek moth situation in Ontario, Jean-François Landry for helping put together the non-target test list and Ana Maria Farmakis for assisting with the upkeep of the cultures at quarantine. Finally, a very special thank you goes to Emma Hunt for your patience when things got busy and for your interest when I (frequently) needed to run my ideas by somebody.

This research was supported by the Improved Farming Systems and Practices Initiative and a Canada Graduate Scholarship from NSERC. Further in-kind contributions were provided by CABI Europe-Switzerland and Agriculture and Agri-Food Canada. 


\section{Table of Contents}

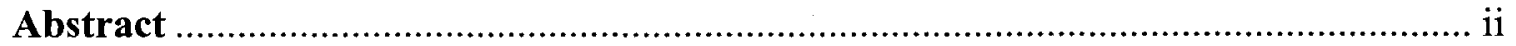

Acknowledgements ............................................................................................ iii

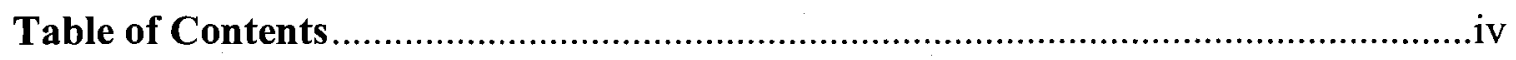

List of Tables.................................................................................................. viii

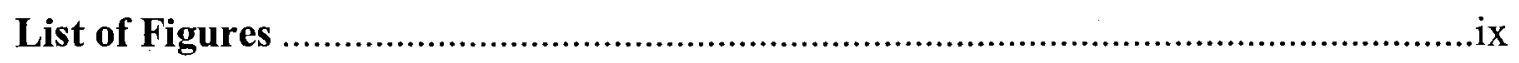

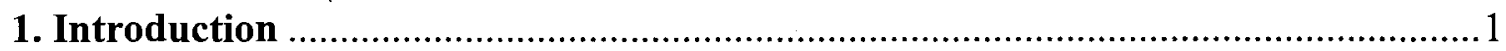

1.1 Biological Control of Insect Pests .................................................................

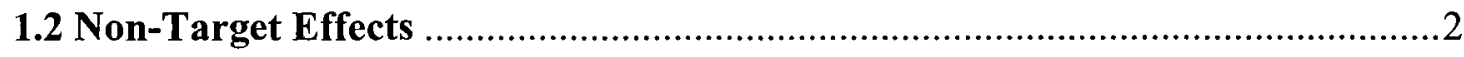

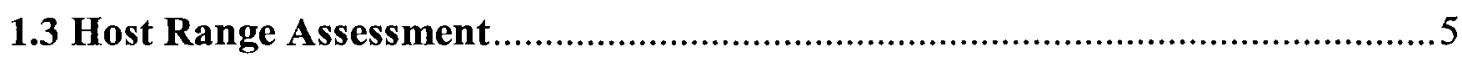

1.4 Effect of Parasitoid State on Host Range Expression ........................................

1.5 Research Objectives and Rationale.....................................................................11

2. Comparative Life Tables of Leek Moth, Acrolepiopsis assectella (Zeller)

(Lepidoptera: Acrolepiidae), in its Native Range ........................................................13

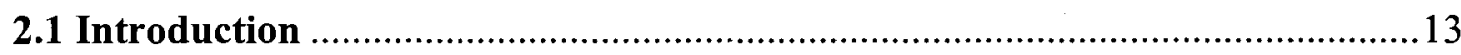

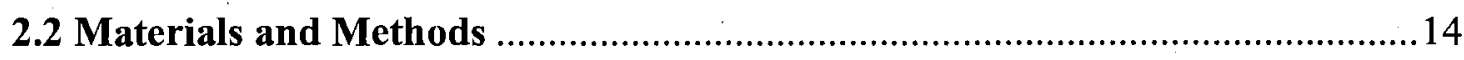

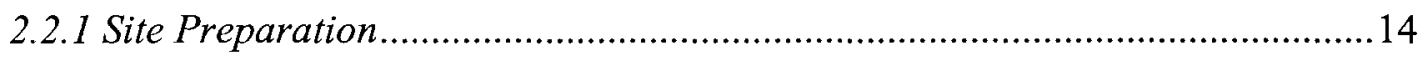

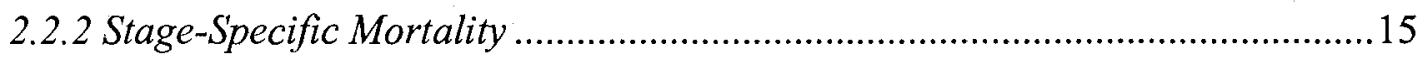

2.2.3 Natural Enemy Impact .............................................................................17

2.2.4 Potential and Realised Fecundity ...............................................................18

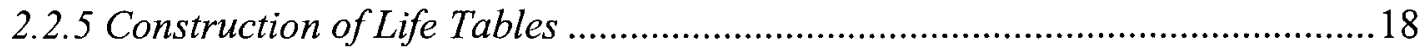

2.2.6 Key Factor Analysis .................................................................................. 19

2.2.7 Data Analyses ......................................................................................20

2.2.8 Field Exploration for Parasitoids .................................................................20

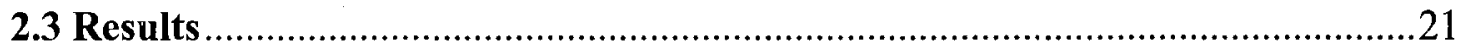

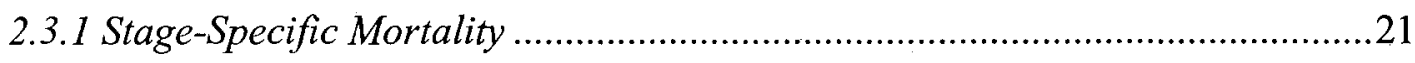

2.3.2 Natural Enemy Impact ............................................................................22

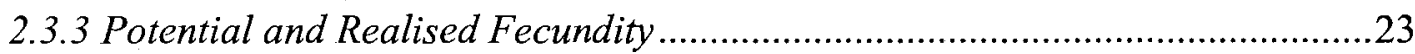

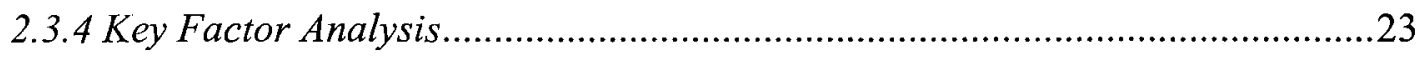

2.3.5 Field Exploration for Parasitoids ...............................................................24

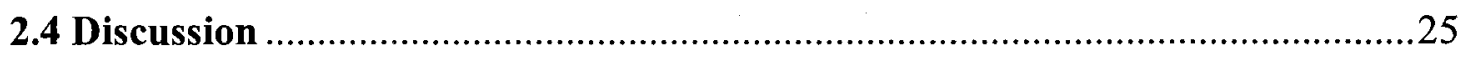


3. Cold Tolerance and Overwintering Strategy of Diadromus pulchellus

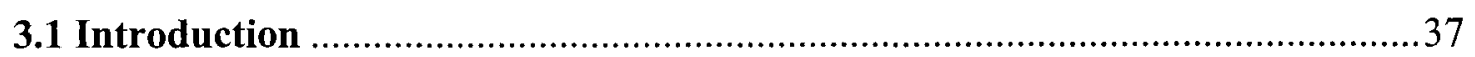

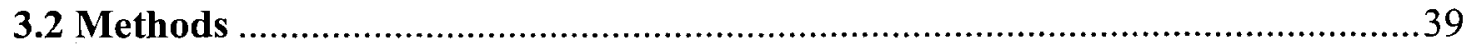

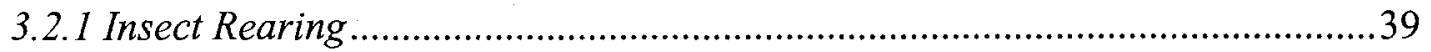

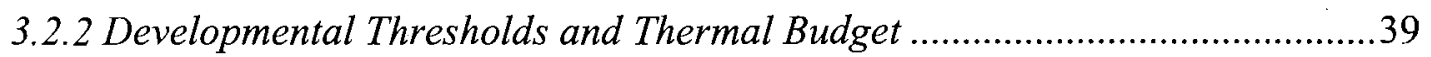

3.2.3 Effect of Variable Durations at Cool Temperatures on

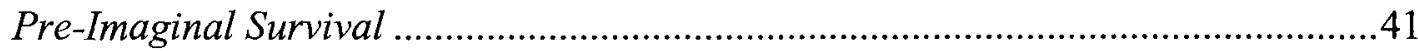

3.2.4 Pre-Imaginal Development under Autumn Conditions ....................................41

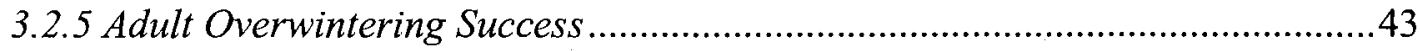

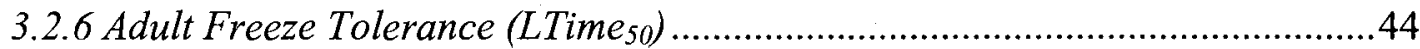

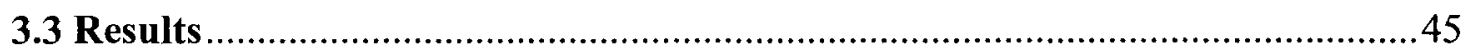

3.3.1 Developmental Thresholds and Thermal Budget .......................................45

3.3.2 Effect of Variable Durations at Cool Temperatures on

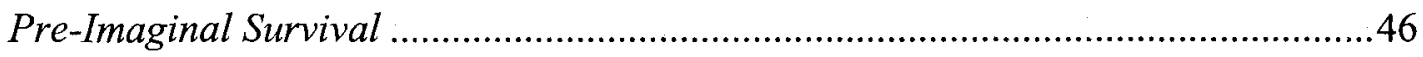

3.3.3 Pre-Imaginal Development under Autumn Conditions .................................47

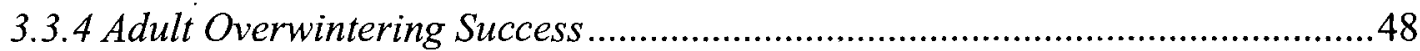

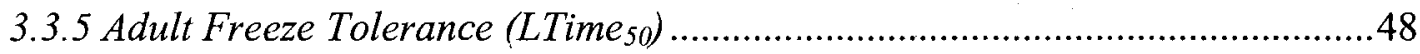

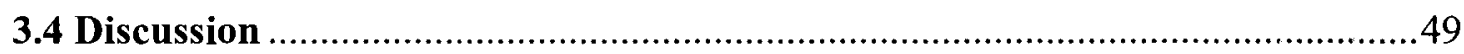

4. Effect of Physiological State and Environment on a Parasitoid's Motivation to

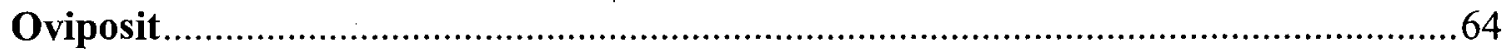

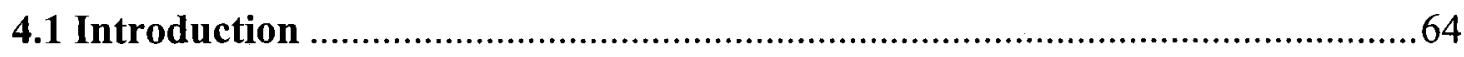

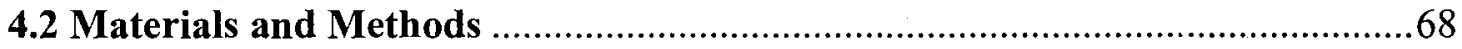

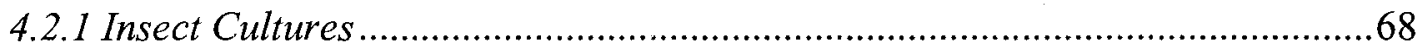

4.2.2 Effect of Host Age on Suitability for Parasitism ...........................................68

4.2.3 Effect of Parasitoid Physiological State and Test Environment

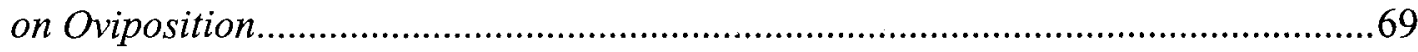

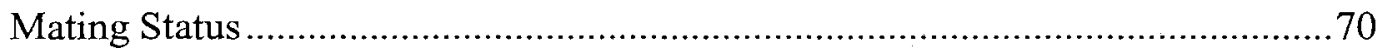

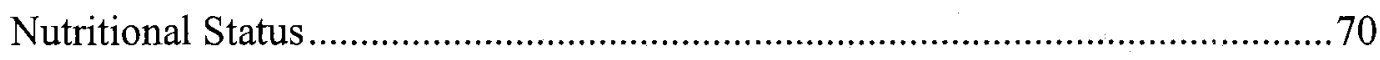

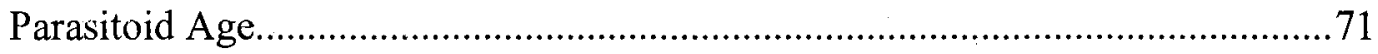

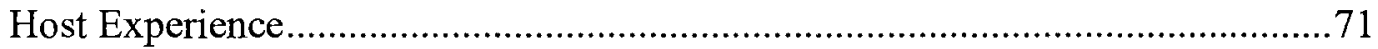

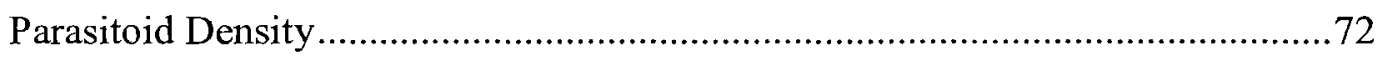




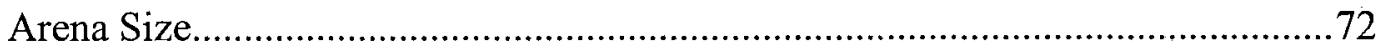

Presence of Host Plant Cues...............................................................................

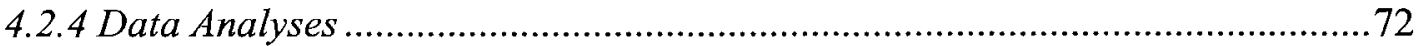

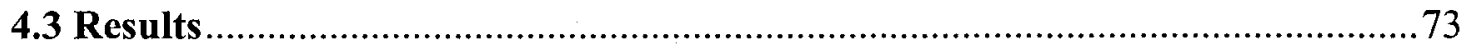

4.3.1 Effect of Host Age on Suitability for Parasitism.............................................73

4.3.2 Effect of Parasitoid Physiological State and Test Environment

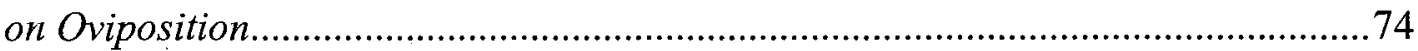

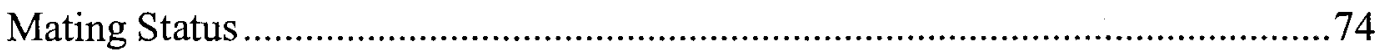

Nutritional Status ......................................................................................

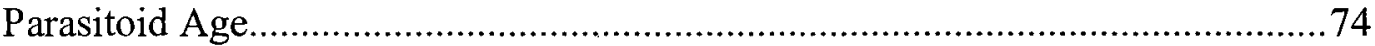

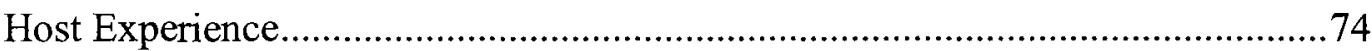

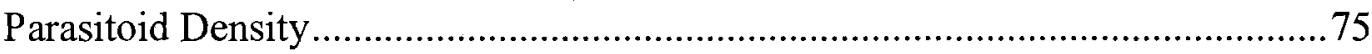

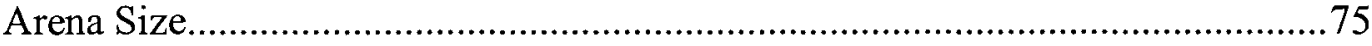

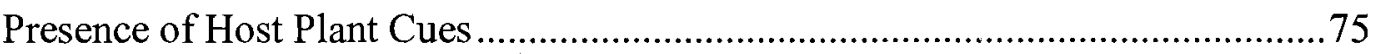

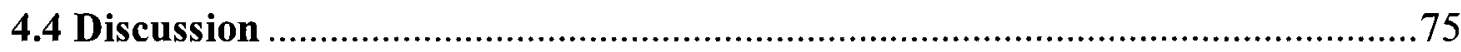

5. Does Parasitoid State Affect Host Range Expression? A Test .................................8

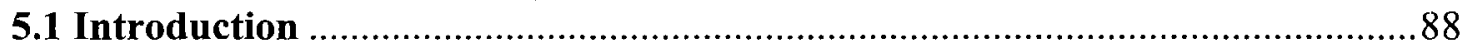

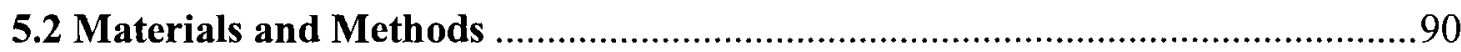

5.2.1 Selection of Non-target Species ......................................................................90

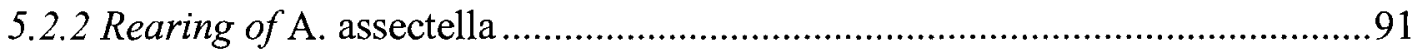

5.2 .3 Rearing of Non-targets ....................................................................................91

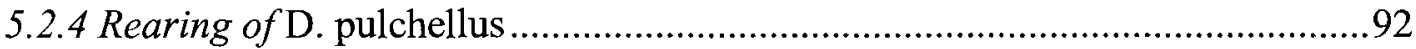

5.2.5 Simultaneous, No-choice, Black-box Test ....................................................93

5.2.6 Choice Tests Between Acrolepiopsis assectella and Plutella xylostella...........95

Direct Choice Test ............................................................................................... 95

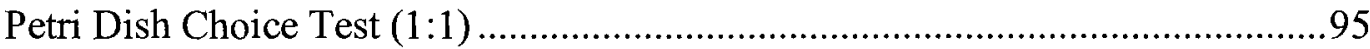

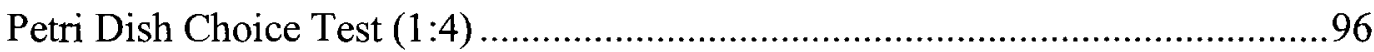

Cage Choice Test.................................................................................................96

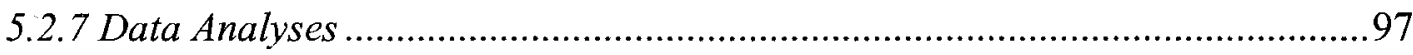

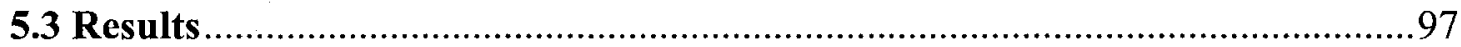

5.3.1 Simultaneous, No-choice, Black-box Test ……………………….................97 
5.3.2 Choice Tests Between Acrolepiopsis assectella and Plutella xylostella...........99

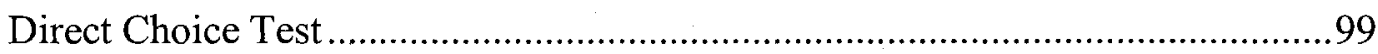

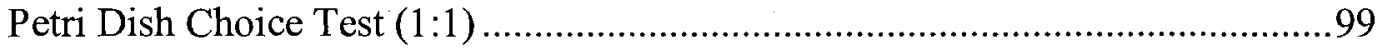

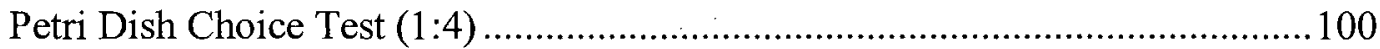

Cage Choice Test ................................................................................... 100

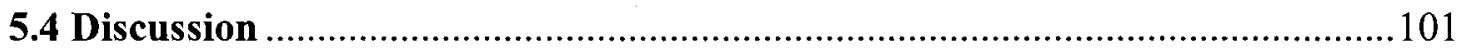

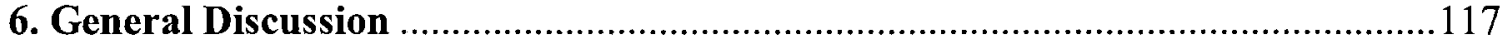

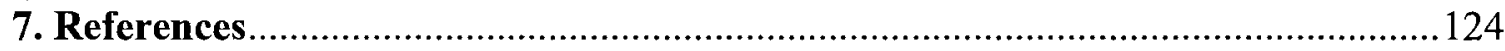




\section{List of Tables}

Table 2-1. Life table for the third generation of leek moth, Acrolepiopsis assectella. Data are pooled from three sites in Switzerland in 2004. $l_{x}=$ number entering stage; $d_{x}=$ number dying in stage; $q_{x}=$ apparent mortality; $m_{x}=$ marginal mortality.

Table 2-2. Life table for the third generation of leek moth, Acrolepiopsis assectella. Data are pooled from three sites in Switzerland in 2005. $l_{x}=$ number entering stage; $d_{x}=$ number dying in stage; $q_{x}=$ apparent mortality; $m_{x}=$ marginal mortality.

Table 2-3. Life table for the third generation of leek moth, Acrolepiopsis assectella. Data are pooled from three sites in Switzerland in 2006. $l_{x}=$ number entering stage; $d_{x}=$ number dying in stage; $q_{x}=$ apparent mortality; $m_{x}=$ marginal mortality.

Table 2-4. Key factor analysis of mortality factors affecting Acrolepiopsis assectella populations at three sites between 2004 and 2006 using regression techniques as described by Podoler \& Rogers (1975). $k_{1}=$ egg; $k_{2}=$ establishing neonates; $k_{3}=$ combined larval instars; $k_{3(1)}=1^{\text {st }}$ instar; $k_{3(2)}=2^{\text {nd }}$ instar; $k_{3(3)}=3^{\text {rd }}$ instar; $k_{3(4)}=4^{\text {th }}$ instar; $k_{3(5)}=5^{\text {th }}$ instar; $k_{4}=$ pupa; $k_{5}=$ lost fertility. ......................... 34

Table 4-1. List of factors tested for their effects on the motivation of Diadromus pulchellus Wesmael to oviposit into its leek moth hosts. Each factor consists of two levels, one of which is considered to be the "ideal state" ("State 1" column). The ideal state is the treatment level predicted, based on foraging theory and empirical studies, to result in the greatest motivation to oviposit. Therefore, "State 1" treatments are expected to show higher rates of host morality and offspring production than the "State 2 " treatments. ................................. 84

Table 5-1. List of non-targets and their respecitve host plants for Diadromus pulchellus host specificity testing. 


\section{List of Figures}

Figure 2-1. Survivorship curves for immature Acrolepiopsis assectella at three commercial leek production sites from 2004 to 2006 (thin solid lines = Site A; dashed lines $=$ Site $B ;$ dotted lines $=$ Site $C ;$ bold line $=$ mean survivorship $) . \ldots . .35$

Figure 2-2. Comparison of stage-specific Acrolepiopsis assectella mortality between caged (white bars) and uncaged (black bars) infested leeks. Error bars show 1 S.E. Asterisks denote life stages where the exclusion treatment significantly affected mortality (Wilcoxon paired-sample test: $* P<0.05 ; * * * P<0.001$ ).

Figure 3-1. Recorded temperatures from late September to early March inside a shaded and open-ended poly-ethylene tunnel used for overwintering trials with both immature and adult Diadromus pulchellus Wesmael. Bold line represents daily means while grey lines show daily minimum and maximum temperatures. Horizontal dashed line shows the freezing point. ..............................55

Figure 3-2. Emergence rates (\% \pm S.E.) of Diadromus pulchellus Wesmael reared from egg to adult at different constant temperatures. The number of replicates per temperature treatment is indicated at the base of each column. Columns with different letters have significantly different means. 56

Figure 3-3. Development rate of Diadromus pulchellus Wesmael reared from egg to adult at different constant temperatures. Line is fitted with simple linear regression (including only mid-range data points: 15 to $27.5^{\circ} \mathrm{C}$ ). Through extrapolation, the lower temperature threshold is calculated as $7.3^{\circ} \mathrm{C}$.

Figure 3-4. Percentage of parasitised pupae ( $\% \pm$ S.E.) yielding healthy Diadromus pulchellus Wesmael offspring following varying durations ( 0 to 10 weeks) at $3^{\circ} \mathrm{C}$, $8^{\circ} \mathrm{C}$ and $12^{\circ} \mathrm{C}$. All exposure durations for each temperature treatment were replicated 6 times.

Figure 3-5. Emergence rate of Diadromus pulchellus Wesmael adults during a five-month treatment period as a function of the date of parasitism. Each pupal cohort was placed in either the outdoor treatment or the $20^{\circ} \mathrm{C}$ control immediately after exposure to parasitoids at room temperature. The percent emergence includes all 
parasitoids that emerged from a particular host cohort over the course of the entire winter treatment. .......59

Figure 3-6. Survival (\% \pm S.E.) of female and male Diadromus pulchellus Wesmael held for 3 months under different overwintering treatments. The " $3,1,6^{\circ} \mathrm{C}$ " treatment consisted of constant $3^{\circ} \mathrm{C}$ for four weeks, then constant $1^{\circ} \mathrm{C}$ for two weeks and then constant $6^{\circ} \mathrm{C}$ for the final six weeks. Each treatment was replicated 5 times for both males and females. Columns with different letters are significantly different from one another.

Figure 3-7. Survival ( $\% \pm$ S.E.) of male and female Diadromus pulchellus Wesmael adults subjected to varying durations of exposure to $-12^{\circ} \mathrm{C}$ to determine the time required to kill $50 \%$ of a cohort. Freeze trials were run with parasitoids (a) reared from egg to adult under summer conditions $\left(22 \pm 2^{\circ} \mathrm{C}\right)$ (replicated 4 times) as well as (b) those emerging from pupae that had been placed outside for the overwintering trial (single replicate only). Parasitoids in the "control" treatment were kept at constant $12 \pm 2^{\circ} \mathrm{C}$.

Figure 3-8. Survival $(\% \pm$ S.E.) of male and female Diadromus pulchellus Wesmael subjected to varying durations of exposure to $-4^{\circ} \mathrm{C}$ to determine the time required to kill $50 \%$ of a cohort. All trials represented here were run with parasitoids reared from egg to adult under summer conditions $\left(22 \pm 2^{\circ} \mathrm{C}\right)$. Each exposure duration was replicated 7 times.

Figure 3-9. Survival ( $\% \pm$ S.E.) of male (single replicate only) and female (replicated 3 times) Diadromus pulchellus Wesmael subjected to varying durations of exposure to $-4^{\circ} \mathrm{C}$ to determine the time required to kill $50 \%$ of a cohort. All trials represented here were run with parasitoids reared from egg to adult under natural autumn and winter conditions.

Figure 4-1. Effect of host age on (a) emergence rates $(F=100.985 ; P<0.001)$ and (b) development times $(F=6.037 ; P<0.001)$ of Diadromus pulchellus Wesmael. ...85

Figure 4-2. Number of (a) offspring produced and (b) hosts killed by Diadromus pulchellus Wesmael females under varying physiological states (mating: virgin $(\mathrm{n}=$ $35)$, mated $(\mathrm{n}=31)$; nutrition: sucrose $(\mathrm{n}=32)$, water $(\mathrm{n}=33)$; age: 3 -day-old $(\mathrm{n}=$ 38), 10-day-old $(\mathrm{n}=45)$; experience: naïve $(\mathrm{n}=54)$, experienced $(\mathrm{n}=49))$. 
Treatments with white bars were predicted to result in greatest motivation to oviposit. ${ }^{* *} P<0.01 ; * * * P<0.001$. . .86

Figure 4-3. Number of (a) offspring produced and (b) hosts killed by Diadromus pulchellus Wesmael females under varying test environment conditions (parasitoid density: single parasitoid $(n=39)$, three parasitoids $(n=39)$; cage size: Petri dish ( $n$ $=34)$, BugDorm cage $(n=34)$; host plant presence: present $(n=35)$, absent $(n=$ 39)). The values shown for the high parasitoid density treatment are totals from all three parasitoids. Treatments with white bars were predicted to result in greatest motivation to oviposit. ${ }^{* * *} P<0.001$.

Figure 5-1. Effect of Diadromus pulchellus Wesmael female age on (a) the percentage of non-target hosts killed (i.e., failing to yield a non-target adult) and (b) the percentage of hosts yielding parasitoid adults in no-choice trials. The number of tested females for each treatment is given in parentheses at the base of each column. Parasitoid age did not have a significant effect on host mortality or offspring emergence.

Figure 5-2. Percentage of no-choice trials in which non-target and target hosts were killed (i.e., failing to yield a moth adult) by (a) 3-day-old and (b) 10-day-old Diadromus pulchellus Wesmael females. Non-target species shown are Acrolepiopsis incertella (Chambers), Plutella xylostella (Linnaeus) and Plutella porrectella (Linnaeus), while the target is Acrolepiopsis assectella (Zeller). The number of trials for each treatment is shown in parentheses beneath the columns. ${ }^{*} \mathrm{P}<0.05 ; * * * \mathrm{P}<0.001$. .111

Figure 5-3. Percentage of no-choice trials in which non-target and target hosts yielded parasitoid offspring following exposure to (a) 3-day-old and (b) 10-day-old Diadromus pulchellus Wesmael females. Non-target species shown are Acrolepiopsis incertella (Chambers), Plutella xylostella (Linnaeus) and Plutella porrectella (Linnaeus), while the target is Acrolepiopsis assectella (Zeller). The number of trials for each non-target is shown in parentheses beneath the columns. $* * * \mathrm{P}<0.001$.

Figure 5-4. Percentage of Petri dish choice trials in which at least one non-target or target host was killed when a 3- or 10-day-old Diadromus pulchellus Wesmael female 
was offered (a) one Acrolepiopsis assectella (Zeller) pupa with one Plutella xylostella (Linnaeus) pupa or (b) one $A$. assectella pupa with four $P$. xylostella

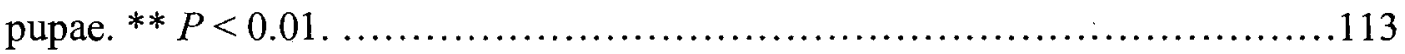

Figure 5-5. Percentage of Petri dish choice trials in which at least one non-target or target host yielded parasitoid offspring when a 3- or 10-day-old Diadromus pulchellus Wesmael female was offered (a) one Acrolepiopsis assectella (Zeller) pupa with one Plutella xylostella (Linnaeus) pupa or (b) one A. assectella pupa with four $P$. xylostella pupae. ${ }^{* *} P<0.01 ;{ }^{* *} P<0.001$.

Figure 5-6. Percentage of cage trials in which at least one Plutella xylostella (Linnaeus) or Acrolepiopsis assectella (Zeller) were killed by (a) 3-day-old and (b) 10-day-old Diadromus pulchellus Wesmael females. When only non-target hosts were present, there were four P. xylostella pupae. In trials with both non-target and target hosts, there were two $P$. xylostella and two A. assectella pupae. 115

Figure 5-7. Mean number of hosts ( \pm S.E.) killed by Diadromus pulchellus Wesmael females in cage trials. When only non-target hosts were present, there were four Plutella xylostella (Linnaeus) pupae. In trials with both non-target and target hosts, there were two P. xylostella and two Acrolepiopsis assectella (Zeller) pupae. In trials with two host species, counts of killed hosts include both species. Neither parasitoid age nor the presence of the target host had a significant effect on the number of hosts killed in a trial $(P>0.05)$. 


\section{Introduction}

\subsection{Biological Control of Insect Pests}

Agriculture is affected globally by the presence of plant, animal and pathogen pests. Cramer (1967) estimated that $34.9 \%$ of food, fiber and cash crops were lost due to pests that year while Oerke et al. (1994) calculated losses of $42.1 \%$ to food and cash crops in the period 1988-1990. Both studies found animal pests to be the largest contributors to crop damage. In many, if not most, countries today, the majority of pest management efforts target exotic species that have invaded cultivated and natural ecosystems following either accidental or intentional introduction. Well known examples of problematic alien arthropod pests in Canada include the diamondback moth, Plutella xylostella (Linnaeus) (Lepidoptera: Plutellidae), the cabbage seedpod weevil, Ceutorhynchus obstrictus Marsham (Coleoptera: Curculionidae) and the emerald ash borer, Agrilus planipennis Fairmaire (Coleoptera: Buprestidae). Invasive herbivorous insects such as these, if left unchecked, can have devastating impacts on the Canadian agriculture and forestry industries.

Chemical pesticides are currently one of mankind's most ubiquitously used weapons to combat the incessant threat that insect pests pose to our agricultural productivity. However, despite the high levels of pest control that result from insecticide use, this chemical approach has been gradually losing favour in many societies. Since the time of Rachel Carson's seminal book, Silent Spring (1962), there has been a growing awareness of the negative aspects of pesticides, including pesticide resistance in insect populations, toxicity to "bystander" species, residual effects and bioaccumulation in the food chain. This concern over pesticides has been a major driving force behind research into alternative and sustainable methods for crop protection. "Integrated Pest Management" (IPM) is now a common practice in agriculture and describes the strategy of using an array of complementary pest control methods (e.g., biological, chemical, cultural and mechanical). Within the IPM framework, natural enemies of pests can play an important role. Biological control, the use of living organisms to reduce pest populations, is not a novel tool in our pest management arsenal. It is a centuries-old 
activity that has been used to target animal, plant, nematode, fungal, or bacterial pests (Cook and Baker, 1983; Bellows and Fisher, 1999).

Biological control of arthropod pests can take many forms, depending on the origin of the agent, its relationship with the pest and the role played by the people involved. Conservation biological control involves the manipulation of the invaded environment, such as providing nectar sources and shelters, to attract and retain a diversity of natural enemies (Barbosa, 1998). Inundative biological control is a method whereby an infested area is flooded with mass-produced agents. These agents will have an immediate impact on the pest population, but their impact lasts only a single generation (Eilenberg et al., 2001). Inoculative biological control is similar, however these agents are expected to establish for a few generations, but not permanently (Eilenberg et al., 2001). When an exotic agent is released with the intention of becoming permanently established in the environment, this is called classical biological control. This often involves selecting natural enemies that have co-evolved with the pest in its native range and if such agents are effective, they can provide long-lasting suppression of the pest. One less common

form of biological control is called new association, or neo-classical, biological control (Hokkanen and Pimentel, 1989). This approach involves introducing an exotic agent to control a pest, either native or exotic, that the agent has not co-evolved with but is capable of attacking. This thesis deals exclusively with arthropod pests and the classical biological control approach. Furthermore, while predators, parasitoids, nematodes, fungi, and bacteria are equally legitimate agents of biological control, the following research will focus on parasitoids, the most commonly used organisms for classical biological control.

\subsection{Non-Target Effects}

There is a long history of discussion on the specific attributes that make natural enemies effective in biological control (Flanders, 1947; Doutt and DeBach, 1964; Greathead, 1986; Waage, 1990; Gurr et al., 2000). Although there has always been a subdued awareness of the risks associated with arthropod biological control (Perkins, 1897; Thompson, 1913; Schaffner, 1934), it is only much more recently that this has 
grown into a strong concern for possible negative effects (Howarth, 1991; Babendreier $e t$ al., 2006). For nearly a full century there was a lack of hard evidence of environmental impacts and biological control was considered a "safe" technology, especially when compared to chemical control options (Batra, 1982; DeBach and Rosen, 1991). Over the last 30 years, more refined risk assessments and pre- and post-release studies have suggested that biological control agents do indeed have the potential to disrupt native species populations (Howarth, 1983; Pimentel et al., 1984; Ehler, 1990; Howarth, 1991; Barratt et al., 1997; Henneman and Memmott, 2001; Elkinton and Boettner, 2004; Johnson et al., 2005; Barrat et al., 2006).

Despite continuous advances in biological control theory (e.g., Waage, 1990; Hawkins et al., 1993; Murdoch and Briggs, 1996; Hawkins and Cornell, 1999; Barlow et al., 2004) and application (e.g., Caltagirone, 1981; Bellows et al., 1992; Gariepy et al., 2005), this management approach has become heavily encumbered by a lack of clarity regarding its potential for direct and indirect environmental impacts. Introductions of exotic natural enemies now endure greater scrutiny from ecologists and environmentalists due to their potential for long-term undesired effects (Tiedje et al., 1989; Louda et al., 2003) and this will inevitably affect progress in this field. The most apparent and frequently discussed risk associated with classical biological control is the potential for introduced agents to attack host species other than the targeted pest species ("non-target effects") (Follett et al, 2000). These so-called non-target effects have formed the central issue in the discussion of the environmental safety of biological control (Louda et al., 2003). Non-targets may include any native species, regardless of their perceived value, or other introduced beneficial species (e.g., weed biological control agents). More difficult to measure and predict are the indirect effects of biological control (Messing et al., 2006). Examples of such effects are intra-guild competition, apparent competition and disease vectoring. While all forms of potential negative impacts should be considered prior to an agent's release, they require different approaches for their evaluation. This thesis deals specifically with the more direct non-target effects relating to parasitoid host specificity.

There is a growing fear that over-regulation of biological control agents will smother this valuable pest management tool. Thus, the practice of classical biological 
control is currently undergoing an intense, international revision in response to a greater demand for harmonisation of methods and regulations (Babendreier et al., 2006). A number of international initiatives (e.g., IPPC, 2005; NAPPO, 2006; OECD, 2003) have emerged in the last decade to formulate guidelines for safe practice, thereby reducing environmental risk and increasing public confidence in biological control. In addition to these guidance documents, two recent books (Van Driesche and Reardon, 2004; Bigler et al., 2006) describe useful methods for biological control risk assessments, with particular emphasis on host range tests. The practice of host range testing is still a developing facet of arthropod biological control and, in some cases, the assumptions that underlie the recommendations in these two books require verification.

Ironically, certain attributes of classical biological control that make it a theoretically superior management strategy are, at the same time, attributes which introduce risk to its application. The self-sustaining nature and permanency of classical biological control is an ideal feature for pest management (DeBach and Rosen, 1991) because it translates to reduced effort on the part of plant protection officials. Both insecticide application and biological control agent production/release are costly, but while chemical use must continue indefinitely, parasitoid releases can be halted once establishment is observed. The concept of permanent establishment, however, is only reassuring if the introduced agent fills a very specific niche in its new environment and interacts exclusively with the target pest. Permanent establishment would be disastrous if, during post-release studies, the introduced agent is found to have population level effects on native non-targets or competitors (e.g., Obrycki et al., 2000). An additional problem to the irreversibility of classical biological control is the tendency for biological control agents to disperse from the target habitat into surrounding areas. Some agents have been recovered far from release sites (e.g., Henneman and Memmott, 2001) and an increasing distribution can lead to more frequent interaction with indigenous floral and faunal communities.

A second feature of classical biological control thought to be desirable is the ability of specialised natural enemies to co-evolve with their host species. The adaptability conferred on parasitoids and predators by their own genetic variability is considered to be an effective tool in preventing the development of resistance in arthropod pests (e.g., 
Barclay, 1996). While a number of insect pests have shown rapidly evolved resistance to several commercially available pesticides (Ankersmitt, 1953; Knight and Norton, 1989), the so-called "evolutionary arms race" between pests and their natural enemies is thought to entail more restricted and gradual genotype frequency changes. On the negative side of the adaptability issue is the potential for an agent to begin exploiting a non-target species that it did not attack in pre-release trials (Gould; 1979; Howarth, 1991). A number of genetically based host switches in insects, mites, and pathogens in both natural and applied settings have been documented in the last five decades (Dennill et al., 1993; Secord and Kareiva, 1996). However, examples of evolving host ranges in insect parasitoids used for classical biological control are either extraordinarily rare or absent in the literature (Louda et al., 2003). Unfortunately, scenarios of natural enemy evolution and host shifts are generally not testable and their consideration would change risk analysis from difficult to virtually impossible.

\subsection{Host Range Assessment}

When a biological control agent does attack a non-target species, it is generally not the result of sudden evolutionary change so much as pre-adaptation and ecological opportunity. This means that classical biological control agents demonstrating polyphagy or oligophagy after introduction likely exhibited similar generalist foraging behaviour in their native ranges or could have, given the right conditions. If this were the case in previous biological control initiatives that resulted in non-target effects, then pre-release studies should have produced data suggesting such species were not safe for introduction. The problem, however, is that host specificity tests are only now becoming a ritual component of release programmes. In Europe, for example, only $1.5 \%$ of entomophagous biological control agents introduced before 1999 were assessed for host specificity prior to release (Lynch et al., 2001). Therefore, much of the environmental damage that biological control might have caused was the outcome of pest management programmes that based their selection of natural enemies solely on efficacy data and likelihood of establishment success (see Frank, 1998 for argument). Among the thousands of arthropod biological control agents introduced to new areas in the last century, many were known 
generalists. The general recommendation today is that such polyphagous species should be avoided since they carry a greater chance of direct and indirect nontarget effects (Louda et al., 2003; but see Nechols et al., 1992). Furthermore, the host ranges of parasitoids are generally considered to be more specialised than those of predators because parasitoids' life histories are more intimately tied to those of their hosts (Price, 1980). Hence, predators have played a more minor role as classical biological control agents (Greathead and Greathead, 1992).

One critical problem with avoiding the use of generalist natural enemies in biological control is the paucity of reliable information regarding parasitoid host ranges in natural ecosystems. Few natural enemies appear to be strictly monophagous (Zwölfer, 1971) and despite decades of observations, there are likely extremely few parasitoid species for which the entire host range (including potential allopatric hosts) is known. Even without consideration of potentially evolving host ranges, it may never be possible to guarantee that a parasitoid will only attack those host species which have already been shown to support its development. The scientific literature is generally not sufficient for identifying specialist natural enemies and concluding that they are safe for introduction into a novel environment. This is because a lack of evidence for use of a particular host ("negative data") does not necessarily mean that the host is outside the potential host range of the parasitoid in question (Strand and Obrycki, 1996). Nevertheless, the literature is a logical starting point in the process of host range assessment. For instance, agents that have been shown to attack numerous host species in different families can be eliminated whereas parasitoids with very few or no host records other than the target host are often worth investigating. It is then up to the researcher to determine through experimentation whether the apparent specificity is a true characteristic of the parasitoid or if it is merely a false result due to insufficient field sampling.

The most effective way to assess a natural enemy's host range is to expose it to a number of potential host species. The ultimate aim of host specificity tests is to identify a proposed agent's "ecological host range". This is defined as the current and evolving set of host species actually used for successful reproduction in the field (Nechols et al., 1992; Onstad and McManus, 1996). However, directly measuring a parasitoid's ecological (syn. "realised") host range is often not a practical option because of the tremendous effort 
required to obtain sufficiently large samples of non-target species in a particular habitat or region.

Following the lead of weed biological control, which has a significantly longer history of host range assessments for candidate agents (McFayden, 1998; van Lenteren et al., 2006), arthropod biological control programmes began to evaluate the "fundamental host ranges" of candidate agents as recently as the 1990's (except in Australia, where such investigations began a decade earlier). The fundamental (syn. "physiological") host range is the set of host species that can support the development of a parasitoid (Nechols et al., 1992; Onstad and McManus, 1996), regardless of the probability of its attack in nature. One can intuitively assume that the fundamental host range would include more host species than the ecological host range. This is because the stimulus-driven foraging behaviour of parasitoids may prevent contact with a very large proportion of the nontarget species present in the field (Doutt, 1959).

Fundamental host range tests can be very simplified in design and therefore omit habitat- or host plant-related stimuli that a parasitoid would normally employ in locating its hosts. To ensure that a parasitoid encounters a non-target host in the absence of these important search cues, these trials may be run in small arenas such as Petri dishes or cages containing only the agent and non-target specimens. Such simple trials make nontarget testing very practical and facilitate the execution of a large number of trials since there are no space constraints, each arena is easy to prepare, and agent behaviour is more easily observed. This approach has been used by many people investigating parasitoid and predator host specificity (e.g., Babendreier et al., 2003; Casagrande and Kenis, 2004; Causton, 2004; Haye et al., 2005). The simplified arena is perhaps the furthest scenario from a realistic, natural design; however, one argument in favour of it is that presenting a target or non-target species on an inert surface helps to standardise the experimental treatments (Withers and Browne, 2004). So long as host encounters in the test arena are guaranteed, this approach should effectively identify those non-target species that are suitable hosts for the candidate agent, even if the list of acceptable host species may be an exaggeration of the agent's ecological host range. This, according to Withers and Browne (2004), is precisely the goal of the first step of host range testing to avoid false-negative results (i.e., absence of attack on a suitable non-target host (sensu Marohasy, 1998)). 
They argued that more natural experimental conditions should only be employed when false-positive results (i.e., unrealistic parasitoid-host associations (sensu Marohasy, 1998)) are strongly suspected.

Ideally, biological control agents would be tested with all possible non-target species from the area of introduction to evaluate the likelihood of attack by the proposed agent. However, due to the vast diversity of insect herbivores (the main hosts of parasitoid species), this is an impossible feat. Therefore, predictions of host ranges frequently try to make generalisations based on data from a subset of representative nontarget species. A number of researchers have also tried to describe patterns of host usage that emerge from assessing parasitoid phylogeny (Strand and Obrycki, 1996), level of niche specialisation (Strand and Obrycki, 1996), or mode of development (Askew and Shaw, 1986).

The selection of non-target species for testing weed biological control agents has traditionally been based primarily on phylogenetic affinity with the target weed (Wapshere, 1974) and on economic importance (Zwölfer and Harris, 1968). However, Kuhlmann et al. (2006) advised that heavy reliance on only the phylogenetic affinity criterion may not allow the development of a suitable non-target test list for arthropod biological control agents due to a number of important differences between entomophagous and phytophagous agents. These authors instead proposed a system in which strong consideration is also given to ecological similarity (e.g., shared habitat or feeding niche) between non-target species and the target pest since parasitoids will sometimes accept hosts based primarily on features of the microhabitat (e.g., Duan and Messing, 1996, 1997; Duan et al., 1997).

As in weed biological control, special consideration should also be given to "safeguard species", which have economic value or are endangered. Unfortunately, locality or phenology data are typically insufficient to obtain specimens of rare species for host specificity tests. In at least two instances, the failure to obtain a rare and potentially at-risk non-target species obstructed releases of the proposed agent in the region where the rare non-target was believed to exist (Sands and Van Driesche, 2000; Sands and Liebregts, 2005). On the other hand, there are arthropod biological control examples in which inaccessible non-targets were simply ignored prior to release of an 
agent (Coombs, 2004). Perhaps the only solution for extremely rare non-target species is to obtain an estimate of parasitism risk by testing a proxy species from the same taxonomic group.

Once a general non-target test list has been composed, it is reduced by applying a number of filters, for instance, removing any species that do not overlap spatially or temporally with the target pest, are morphologically different from the target, or are difficult to obtain and rear. The end product is a workable test list where priority can be given to species that have the most biological and ecological attributes in common with the target pest. This revised list can later be modified if new information suggests that additional species ought to be included in the tests.

\subsection{Effect of Parasitoid State on Host Range Expression}

In much the same way that the size of a test arena or the presence of habitat-related stimuli may influence a parasitoid's foraging decisions or readiness to oviposit, so, too, can the physiological and informational states of the parasitoid (Roitberg, 2000; Withers and Browne, 2004). Examples of physiological states include mating status (virgin vs. mated), egg load (egg-limited vs. egg-rich), and hunger (fed vs. starved). The informational state of an agent refers to its knowledge of its environment as well as its own condition. The informational state may vary with respect to previous experiences with the host (naïve vs. experienced) or detection of competing parasitoids in the vicinity (solitary vs. aggregated). Moreover, changes in an agent's physiological state may affect its informational state. In many cases, two or more factors, such as parasitoid age and egg load, may be highly correlated and not clearly separable (Withers and Browne, 2004); therefore, an assessment of the effects of a particular factor on host acceptance must be conducted with caution.

Withers and Browne (2004) further argued that if a biological control agent's responsiveness to its target host can be affected by variation in endogenous and exogenous factors, then this variation may also influence the agent's host specificity. Building upon that argument, these authors made several recommendations on ways in

which candidate agents might be treated in order to maximise the likelihood of attack on 
unfamiliar hosts and thus avoid false-negative results. They developed each of their suggestions by reviewing the literature for observable patterns in the effects of parasitoid states on oviposition behaviour. In many cases, a specific change in parasitoid condition appeared to have a unidirectional effect on a parasitoid's "motivation to oviposit". For example, among haplodiploid parasitoid species, mated females have demonstrated an increase in foraging activity compared to virgin conspecifics (Michaud and Mackauer, 1995; Para et al., 1996). Withers and Browne (2004) therefore advocated using only mated agents in host specificity trials. On the other hand, certain factors such as prior experience with the host may have less predictable effects on oviposition (e.g., Bueno et al., 1993; Kitt and Keller, 1998).

Although Withers and Browne (2004) drew on a sizeable collection of literature for their host range testing guidelines, none of the publications they cited has explicitly tested the effect of parasitoid state on the outcome of a host range test. Instead, their suggestions were based on the following series of assumptions: (1) a change in physiological state that modifies one behavioural parameter, such as responsiveness to odours, would also induce a change in the parasitoid's motivation to oviposit; (2) the direction and magnitude of change in one behavioural parameter will translate to a roughly equivalent transition in the probability of an oviposition event occurring; and (3) an increased readiness to oviposit in the target host will translate to a similar increase in willingness to oviposit in non-target hosts. While these assumptions may be true for some entomophagous insects, there is a strong possibility that endogenous and exogenous factors do not affect all species equally. Although it is desirable to have more standardised protocols for host range testing, some classical biological control programmes cannot afford to be further handicapped by experimental rules based on "probable" hypotheses. Before adopting generalised testing procedures such as those proprosed by Withers and Browne (2004), the underlying assumptions must first be found scientifically sound.

In arthropod biological control programmes, parasitoid condition has rarely been considered a potentially significant factor affecting host specificity. This is illustrated by the fact that authors of past studies often have not described the conditions under which agents were reared and held prior to testing. Five examples of the most commonly 
described conditions for rearing and testing are mating status, nutritional status, age and host experience of the agent at the time of testing as well as the number of agents tested together in a single replicate (i.e., agent density). A review of the Materials and Methods from 72 host specificity tests on parasitoids (1984 to 2007) revealed that only $27(37.5 \%)$ of them explicitly described the status of all five conditions listed above. Furthermore, there has been no apparent increase in the frequency with which this type of information is reported in recent years. One could imagine that agent physiological and informational state will feature more prominently in host range test discussions if these factors are found to significantly influence an agent's response to non-target hosts.

\subsection{Research Objectives and Rationale}

Some of the questions that arise from Withers and Browne's (2004) guidelines are addressed in this thesis using a host-parasitoid model system. The insects to be studied are of significant importance as they are currently under investigation for a classical biological control programme in Canada. The pest (host) species is leek moth, Acrolepiopsis assectella (Zeller) (Lepidoptera: Acrolepiidae) while the agent is the pupal parasitoid Diadromus pulchellus Wesmael (Hymenoptera: Ichneumonidae). The research presented in this thesis is therefore multi-faceted, with two principal objectives involving the host-parasitoid model system: (1) to explore the potential for classical biological control of the invasive leek moth in North America and (2) to test the fundamental assumptions in Withers and Browne's (2004) recommendations for host range testing.

Chapter 2 describes a life table analysis of leek moth in its native range. A major assumption behind classical biological control is that insects and weeds become severe pests when introduced to novel environments because they leave behind the natural enemies that normally suppress their populations (Keane and Crawley, 2002). When the "enemy release hypothesis" is true for a given pest, there should be evidence of top-down control in its area of origin. Life tables can be useful for calculating the specific contributions of different mortality factors as well as identifying life stages of the pest that appear to be most vulnerable to natural enemies. This chapter also contains a brief explanation of the field surveys conducted in central Europe to obtain parasitoids of leek 
moth. Justification is given for the selection of $D$. pulchellus as the primary candidate for classical biological control in Canada.

Chapter 3 provides a discussion on the suitability of $D$. pulchellus for a Canadian climate. The results from several experiments are interpreted to explore this agent's overwintering strategy and the probability of it surviving winters that tend to be harsher than it experiences in central Europe. A suspected common cause of classical biological control failures is poor climate matching (DeBach and Bartlett, 1964). An introduced agent may have a significant impact on pest densities immediately following release, however its efficacy will be short-lived if it cannot withstand the winter conditions of the new environment. Costly mass-releases of a "doomed" agent may be avoided by investing a small amount of research into an assessment of its cold tolerance prior to its release.

Chapters 4 and 5 address the question, described in the preceding section, of whether an agent's condition and the surrounding environment can have a predictable influence on its probability of accepting non-target host species. Withers and Browne's (2004) assumptions were turned into a working hypothesis which states that a parasitoid, whose motivation to attack its target host is enhanced by a particular physiological or informational state (e.g., mated), will also parasitise non-target species more readily than a parasitoid with the opposing condition (e.g., virgin). Conversely, the null hypothesis is that parasitoid condition and environmental cues will have no impact on host range expression. The specific aim of Chapter 4 was to measure how the oviposition rate of $D$. pulchellus on leek moth was affected by several physiological and environmental factors. The next step, in Chapter 5, was to determine whether manipulation of an agent prior to testing could influence non-target acceptance in host specificity tests. The outcome of host range testing was compared to predictions (i.e., direction and magnitude of effect) based on the results from Chapter 4. 


\section{Comparative Life Tables of Leek Moth, Acrolepiopsis asectella (Zeller) (Lepidoptera: Acrolepiidae), in its Native Range}

\subsection{Introduction}

The leek moth, Acrolepiopsis assectella (Zeller) (Lepidoptera: Acrolepiidae), is a widely distributed, multivoltine pest of Allium spp. (Asparagales: Alliaceae) in Europe (Jary and Rolfe, 1945; Frediani, 1954; Markula, 1981), Asia (Velitchkevitch, 1924) and North Africa (Labeyrie, 1966). Without appropriate control measures, the effects of leek moth on Allium production can be devastating (e.g., Plaskota and Dabrowski, 1986; Nyrop et al., 1989), leading to heavy loss of vegetable and seed products. The larvae are concealed feeders, mining primarily in the leaves and stems of their host plants, but damaging flower buds and even the bulbs under extreme circumstances (Noyes, 1974). Leek moth is best known for its impact on leek production but also commonly attacks onion, shallot, garlic and chive. Following its unintentional introduction into North America and discovery in 1993 (Landry, 2007), leek moth has established itself as a pest of concern in Allium production in eastern Ontario and western Quebec. Because the leek moth is a quarantine pest in the US, where, as of early 2008 , it had not yet been detected, the cross-border movement of Allium products has been partially disrupted (M.-P. Mignault, Canadian Food Inspection Agency, pers. comm.). In addition to its effects on agriculture, leek moth may pose a threat to North American Allium biodiversity. In a recent study, Allison et al. (2007) demonstrated that wild Allium species stimulate leek moth oviposition and can support larval development.

Pheromone trap data from the Ottawa, Ontario region showed population levels up to ten times greater than at sites simultaneously sampled in Switzerland, within its area of origin (Mason et al., 2006; Jenner and Kuhlmann, 2005). This commonly seen disjuncture of pest densities between areas of origin and introduction is often ascribed to the enemy-release hypothesis, whereby introduced species experience increased abundance due to the failure of their co-evolved natural enemies to follow them to the new range (Elton, 1958; Debach and Rosen, 1991; Van Driesche and Bellows, 1996). This hypothesis is built on the foundation that a pest is suppressed, at least in part, by a 
natural enemy community in its area of origin. It therefore follows that assessments of these predator and parasitoid assemblages may lead to the identification of suitable biological control agents.

Exploration for candidate classical biological control agents for leek moth was initiated in 2004. In the same year, a life table study was launched to identify the principal factors that inhibit leek moth population growth in areas of low pest density in central Europe. The primary aim of this study was to build and analyse life tables for leek moth over multiple years in its area of origin to identify key stages of mortality. In addition to quantifying mortality in the 'traditional' insect life stages (i.e., egg, larva and pupa), this study attempts to shed more light on the subject of post-hatch mortality and the factors behind it by measuring the rate of successful establishment of newly eclosed first instar larvae. Although leek moth larvae feed internally, much of the life cycle occurs outside the host plant. Eggs are laid superficially and, prior to pupation, final instar larvae abandon their feeding galleries to spin a cocoon on the outside of the plant. In their comparisons of pre-adult survival in endophytic and exophytic species, Price (1975) and Cornell and Hawkins (1995) observed a trend for greater mortality in the latter. It was therefore anticipated that leek moth field mortality would be greater in the egg and pupal stages than the larval instars. Finally, this chapter explains how candidate classical biological control agents were obtained from leek moth and gives justification for the selection of one species for closer study.

\subsection{Materials and Methods}

\subsubsection{Site Preparation}

The three-year life table study was conducted in commercial organic and integrated production leek fields from 2004 to 2006 . In each year, leek moth mortality was measured simultaneously at three field sites, each from one of three regions in Switzerland. Site A (near Bern, western Switzerland N 46 $59.488^{\prime}$ E $7^{\circ} 7.685^{\prime}$ ) was approximately $120 \mathrm{~km}$ southwest of Site B (near Zürich, north central Switzerland, N $47^{\circ}$ $31.100^{\prime} \mathrm{E} 8^{\circ} 28.521^{\prime}$ ), which in turn was approximately $55 \mathrm{~km}$ southwest of Site C (near Konstanz, northeastern Switzerland N $47^{\circ} 39.003$ E $9^{\circ} 7.131$ '). Five sampling blocks (3 $\mathrm{m} \times 2 \mathrm{~m}$ ) were set up at each field site, two in the crop centre and three along the field 
margins. For each leek moth life stage, there were six infested leeks in each of the five blocks ( $=30$ sampled leeks per field per life stage). To standardise the quality of test plants between sites and years, individual potted leek plants, grown at the CABI EuropeSwitzerland laboratory, were inserted among the commercial leeks in each block. Leek moth undergoes three generations annually in Switzerland. The life table study commenced when pheromone trap counts indicated the start of the third generation. Due to the immensity of this project, it was not possible to conduct the life table study over all three leek moth generations. The final generation was selected for study since it had previously been shown to have the highest levels of attack by parasitoids (Plaskota and Dabrowski, 1986).

\subsubsection{Stage-Specific Mortality}

Although leek moth adults were caught in pheromone traps at all sampling sites, the natural populations were generally too low to even detect infestation. Hence, field mortality was evaluated using artificial populations. This manipulative approach enabled us to standardise the age and density of insects placed on each plant in the field at a given time. All sentinel leek moths were reared on leeks in the laboratory at $20 \pm 3^{\circ} \mathrm{C}$ and 16L:8D and then transferred to the field once they had reached the desired life stage. The pre-imaginal stages were set out in the field in a sequential fashion, beginning with the egg stage, and were timed to match the phenology of natural leek moth populations. Each developmental stage was exposed in the experimental blocks for only the duration of that stage. The aim was to retrieve the immature leek moths from the field just prior to eclosion (eggs and pupae) or during moulting (larvae). The duration of field exposure for a particular life stage was determined by closely monitoring the development of a subset of individuals, held outside at the CABI Centre. The development rate of leek moths in late summer was similar among the three field sites and the CABI Centre. The sentinel leek moths were retrieved from the field when the monitored subset reached the end of its current life stage. The infested leeks were also used only once.

Leek moth eggs were obtained by motivating adult moths to oviposit on 'egg strips', strips of stiff, smooth, green plastic. Pieces of crushed leek leaf were rubbed vigorously over the surface of the egg strips, which were then placed into a cage 
overnight with hundreds of adult moths. The next morning, the egg strips were removed from the adult cage and cut into smaller pieces (approximately $2 \mathrm{~cm}^{2}$ ) containing 10-20 eggs. All malformed eggs were removed. These egg batches were then taken immediately to the field sites and attached, one per plant, to leeks within the blocks. To mimic a natural distribution of leek moth eggs according to Noyes (1974), most egg strips were pinned to the ventral side of the lower leaves with a smaller portion allocated to dorsal surfaces and upper leaves. Upon recovery from the field, the eggs were brought back to the laboratory to observe egg hatch and record survival.

To measure mortality during the neonate establishment phase, a second cohort of eggs was placed in the field as explained above, but with one difference: instead of retrieving these egg strips just prior to egg hatch, they were left in the field for extra time to allow for egg hatch and the subsequent entry of neonate larvae into the leek leaves. These infested leeks were then retrieved and dissected in the laboratory to count the number of first instar larvae that had managed to establish on each plant. Post-hatch mortality was calculated by dividing the number of successfully established neonate larvae by the number of hatched eggs. Henceforth, the use of "neonate" will refer strictly to the pre-feeding phase of wandering and leaf penetration by newly hatched larvae.

The five larval instars were placed in the field sequentially following the egg stage. As the sentinel eggs were recovered from the experimental blocks, they were replaced immediately with first instar larvae. Similarly, when the first instar larvae were removed, the second instar exposure began. This swapping of subsequent life stages continued until the end of the pupal stage. To prepare the first instar cohort, newly eclosed larvae $(<2$ minutes since hatch) were transferred directly onto healthy potted leeks. Cohorts of the second, third, fourth and fifth instars were prepared for field exposure in precisely the same way except that they had to be dissected from their feeding mines before being transferred to the experimental leeks. Only larvae that had recently moulted to the instar of interest, identified by colour and relative size of the head capsule, were used for the life table study. The larval density per plant for each instar was: 5 first instars, 5 second instars, 4 third instars, 3 fourth instars and 3 fifth instars. The transferring of larvae to potted leeks was always done late in the evening, giving the larvae several hours to safely establish feeding mines in the laboratory before the leeks were taken to the field sites the 
next morning. The infested potted leeks were inserted into the experimental blocks among the field leeks and the bases of the pots were dug at least $5 \mathrm{~cm}$ into the soil. In addition, any leaves from surrounding plants that were touching the sentinel potted leeks were clipped to reduce the likelihood of larvae walking off the experimental plants. Each larval instar was left in the field for as many days as necessary to reach the next moult. Retrieving the sentinel larvae consisted of transporting the potted leeks back to the laboratory where they were dissected to obtain all remaining specimens. The surviving individuals were reared on sections of leek leaves in labelled Petri dishes $(9 \mathrm{~cm}$ diameter, $2 \mathrm{~cm}$ depth) to record the emergence of parasitoids.

Mortality in the final immature stage was measured by attaching leek moth cocoons, two per plant, to leeks within the field blocks. The cocoons were collected from the laboratory culture in the morning and taken immediately to the three field sites. Fine insect pins (00 and 000) were used to attach the cocoons to random places on the assigned leeks since leek moth is known to pupate on all parts of the plant (Thibout and Nowbahari, 1987). Preliminary trials had shown that pinning cocoons to leaves had no effect on leek moth survival or oviposition success by parasitoids. As these cocoons were less than 24 hours old they all contained a pre-pupa, which then pupated at some point during the first or second day in the field, depending on temperature. This life stage, hereafter called "pupa", therefore actually includes mortality inflicted during both the pre-pupal and pupal phases. The pupae were retrieved from the field as close to adult eclosion as possible and brought to the laboratory to assess mortality.

\subsubsection{Natural Enemy Impact}

In addition to calculating stage-specific field mortality rates, an exclusion technique was used to evaluate the effect of predators and parasitiods on leek moth survival. For each life stage, all blocks within the three sites were assigned three control (caged) plants and three treatment (exposed) plants. Following infestation with sentinel leek moth individuals, the control leeks were covered with finely woven muslin bags $(0.3 \mathrm{~mm}$ space between threads), which were supported around each plant by a single, parabolic-shaped wire frame. Prior to infesting and covering the control plants, each one was shaken vigourously to dislodge any potential natural enemies that were resting on the plant. At 
the base of the plants, the muslin bags were dug into the soil to completely block access by macroscopic arthropod and vertebrate natural enemies. In contrast, the uncovered plants were fully exposed to all biotic mortality factors. Estimation of impact by natural enemies was done by comparing mortality between caged and exposed leeks.

\subsubsection{Potential and Realised Fecundity}

To construct complete life tables, it was necessary to estimate the fecundity of leek moth females. At the same time that leek moth eggs were placed in the field, oviposition trials were conducted to measure both potential and realised lifetime fecundity. The potential fecundity was determined by placing a newly emerged male and female pair into a Petri dish ( $9 \mathrm{~cm}$ diameter, $2 \mathrm{~cm}$ depth) containing a freshly cut, $4 \mathrm{~cm}$ long leek leaf segment and a piece of dental cotton, hereafter called a 'cotton bud', soaked in a $20 \%$ sucrose solution. The oviposition arenas were kept at $21 \pm 2^{\circ} \mathrm{C}$ and $16 \mathrm{~L}: 8 \mathrm{D}$. All eggs laid in the Petri dish were counted every two to three days, at which time the moth pair was transferred to a new Petri dish with a new leaf disc and cotton bud. A total of 50, 15 and 23 moth pairs were tested in 2004, 2005 and 2006, respectively.

Realised fecundity was measured in 2004, 2005 and 2006 by enclosing male-female pairs with small potted leeks, which were then placed along field margins at the three field sites. These experimental leeks were framed by two perpendicular, parabolic-shaped wires and covered with finely woven muslin bags ( $0.3 \mathrm{~mm}$ space between threads), which were sealed around the base of the pot with elastic bands. These exclusion cages

prevented access to the moths by natural enemies, but exposed the moths to natural light, moisture and temperature levels. Every 3-5 days, the moth pairs were transferred to a fresh potted leek and the eggs laid during the previous days were counted. A total of 30 , 32 and 25 moth pairs were tested 2004, 2005 and 2006, respectively. All fecundity trials continued until the death of the female.

\subsubsection{Construction of Life Tables}

The field mortality data were used to construct survivorship curves and life tables for each year of the study. Whereas the mortality probability for each life stage was calculated from a fresh cohort of a pre-determined number of leek moths, the life tables 
were constructed in a more longitudinal fashion, with a standardised starting number of 1000 eggs to effectively illustrate accumulated mortality.

Since the study design consisted of five discrete sampling blocks per site, these blocks were treated as cluster samples. Mean mortality rates were calculated from the three caged and three exposed leeks in each block. Hence, each field site had five data points for both the caged and exposed treatments. Apparent mortality $\left(q_{x}\right)$ is the ratio of the number dying $\left(d_{x}\right)$ in stage $x$ to the number entering $\left(l_{x}\right)$ that same stage. When multiple mortality factors act simultaneously, the marginal attack rate $\left(m_{x}\right)$ is a useful measure because it calculates the proportion of individuals of a particular stage that would be killed by a single factor if it were acting alone (Bellows et al., 1992). If there is only one identified mortality factor or if the multiple mortality factors operate sequentially without overlap, then the marginal death rate will equal apparent mortality. When factors operate contemporaneously and there is no information on interactions between those factors, the marginal death rate is calculated as: $m_{x}=1-(1-q)^{q / q}$, where $q_{i}$ is the apparent mortality caused by the $i$ th factor while $q$ is the stage mortality rate caused by all factors (Elkinton et al., 1992).

The outcome of a life table can be expressed as the mean realised progeny of a population (i.e., the mean realised lifetime fecundity multiplied by the number of surviving females). A sex ratio of 1:1 was assumed (Noyes, 1974). Lost fertility, potential minus realised fecundity, was incorporated into the life tables to account for the impact that incomplete egg laying has on population change. The inclusion of fecundity estimates is important because it permits calculation of net reproductive rate $\left(R_{0}=\right.$ number of female offspring from a single reproductive female).

\subsubsection{Key Factor Analysis}

Once marginal death rates were determined, $k$-values, calculated as the difference between logarithms of $l_{x}$ before and after the action of each mortality factor (Varley and Gradwell, 1960), were assigned to each mortality factor. This facilitated measuring the contribution of each $k$-value to the generational, or total, mortality $(K)$, which is the sum of all $k$-values in a life table. The $k$-values were additionally used to identify the key mortality factors that appear to be largely responsible for changes in population density 
(Morris, 1959). First, the method described by Varley and Gradwell (1960) was applied, where the total mortality and stage-specific $k$-values were plotted for a set of nine consecutive life tables ( 3 life tables $\times 3$ years). The $k$-value that best tracks total mortality is said to be the key factor. Second, a series of regressions of each $k$-value sequence against $K$ was performed. The individual $k$-value that gave the greatest slope, while maintaining a significant correlation to $K$ was recognised as the key factor (Podoler and Rogers, 1975). While the five larval instars are shown separately in the life tables below, they were pooled for the key factor analysis by taking the sum of their $k$-values.

\subsubsection{Data Analyses}

All statistical analyses were carried out on arcsine square root transformed data using SPSS version 14.0 (SPSS Inc, Chicago, IL). Linear regression analysis was used to test for a correlation between total pre-imaginal development time (exposure duration) and total mortality. Stage-specific mortality rates within each year and female fecundities were compared using one-way ANOVA and the means were separated using the Tukey HSD test. Several of the paired data sets from the exclusion experiment did not fit a normal distribution following transformation; therefore, the comparison of mortality under caged and exposed conditions for each life stage was performed with Wilcoxon paired-sample tests. The significance level was set at $\alpha=0.05$ and all data presented in the tables, figures and text, unless stated otherwise, are untransformed means.

\subsubsection{Field Exploration for Parasitoids}

Leek moth parasitoids were obtained by collecting samples of leek moth larvae and pupae from infested leek, chive and onion fields in Switzerland. Sampling excursions were carried out routinely from mid-April until mid-September in 2004 and 2005 to cover all of the three leek moth generations. Field-collected larvae were reared in the laboratory on small sections of leek leaves in Petri dishes while pupae were stored in clean Petri dishes. All specimens were monitored regularly for parasitoid emergence. Due to low natural populations of leek moth at most of the survey sites, several sentinel potted leeks containing large numbers of eggs, larvae and pupae were also placed along field margins and retrieved a few days later to capture and identify any emerging parasitoids. 
All parasitoids reared from leek moth were sent to European taxonomists for identification.

\subsection{Results}

\subsubsection{Stage-Specific Mortality}

Figure 2-1 shows survivorship curves that were generated for each site from 2004 to 2006 and exhibits a relative consistency in leek moth attrition, with the greatest variation occurring in egg, neonate and pupal mortality. Complete life tables giving stage-specific mortality rates for each year are presented in Tables 2-1, 2-2 and 2-3. Total mortality and fecundity values for each year are derived from three life tables representing the three field sites (data are pooled, site-specific tables are not shown). Total mortality of the immature stages did not vary significantly between 2004 (99.6\%) and 2005 (99.1\%), but dropped slightly in 2006 (96.4\%). This lower total mortality in 2006 was due primarily to higher survival during neonate establishment that year. For instance, the three highest survivorship curves in Figure 2-1 are all from 2006. Inter-site variation was greatest in 2005. Site $\mathrm{C}$ had the highest total mortality rates in all years of the study whereas Site $B$ had the lowest values in 2005 and 2006.

During the course of leek moth development, there were significant differences in stage-specific mortality rates (2004: $F=21.83, P<0.001 ; 2005: F=43.65, P<0.001$; 2006: $F=21.00, P<0.001$ ). In all years of the study, mortality was greatest during the egg, neonate and pupal phases but low for individual larval instars. Egg mortality ranged from $50 \%-70 \%$ and was a consistent key contributor to generational mortality. In both 2004 and 2005, neonate mortality contributed strongly to generational mortality $(22.9 \%$ and $28.0 \%$, respectively) but, as mentioned earlier, this stage suffered less mortality in 2006 (only $9.7 \%$ of generational mortality). Neonate mortality was significantly higher than egg mortality in 2005, but was not significantly different in 2004 and 2006. On an instar-by-instar basis, leek moth larvae appeared to be less vulnerable to mortality factors. It was extremely rare in any of the nine site-specific life tables for mortality in a particular larval instar to surpass that of the non-feeding stages. Among the larval stages, mortality was highest in the early instars. The means for larval mortality averaged over all sites and years are as follows: $\mathrm{L} 1=31.6 \% ; \mathrm{L} 2=29.0 \% ; \mathrm{L} 3=21.9 \%$; $4=11.3 \%$; L5 
$=19.2 \%$. When all instars are combined, total larval mortality was $84.9 \%, 69.0 \%$ and $59.7 \%$ in 2004, 2005 and 2006, respectively. These mortality rates translate to $25.0 \%$, $18.4 \%$ and $18.0 \%$ of generational mortality in each of those years, respectively, and thus make larval death one of the greatest contributors to immature mortality. On average, larval mortality showed a decreasing trend over the three years of the study. In contrast mortality of pupae gave the opposite response such that it contributed twice as much to generational mortality in 2006 than in 2004. Due to this increase in pupal mortality and the drop in neonate death in 2006, pupal mortality replaced the latter as the primary contributor to generational mortality in the final year.

In virtually all cases, leek moth mortality was attributed to unknown causes. While generalist predators have been observed feeding on leek moth eggs and pupae in the field, it was rarely possible to accurately identify the cause of death for these stages. Among the larval instars, very few cadavers were recovered, making disappearance the primary factor behind larval loss. It was not clear what led to the separation of larvae from their host plants and the probability of subsequent survival and successful relocation of a suitable host plant is also not known. There was almost no apparent mortality caused by parasitoids in this life table study. At all three sites and over all three years, no egg or pupal parasitoids were reared from the sentinel leek moth specimens. Although larval parasitism was detected, it consisted of only two parasitised larvae: one second and one third instar. Both incidences occurred on exposed leeks at Site C in 2004 and involved the same parasitoid species, Diadegma chrysostictos (Gmelin) (Hymenoptera: Ichneumonidae).

\subsubsection{Natural Enemy Impact}

Figure 2-2 illustrates the comparison of mortality on caged and uncaged leeks for each of the three years (sites are once again pooled). In most cases, there were no statistically significant differences between treatments even though stage-specific mortality was greater on exposed than on caged leeks in 21 of the 24 comparisons. The pupa was the only stage to have significantly greater mortality on exposed plants in every year. Other stages where significant differences were detected between caged and uncaged plants were the fourth instar in 2004 and neonate and first instar in 2005 . While 
not statistically significant, there were substantial differences in egg stage mortality in 2004 and $2006(P=0.053$ and $P=0.069$, respectively $)$.

\subsubsection{Potential and Realised Fecundity}

Under optimal conditions in the laboratory, leek moth females laid means of 349.8 \pm 10.7 (S.E.), $297.0 \pm 26.9$ and $322.6 \pm 27.0$ eggs in 2004, 2005 and 2006, respectively. These inter-annual differences were not statistically significant $(F=1.19, P=0.311)$. The maximum potential fecundity observed in each year was 519, 429 and 507 eggs, respectively. Oviposition by leek moth under field conditions was substantially reduced. The maximum realised fecundity observed was 271, 264 and 293 eggs, in 2004, 2005 and 2006 , respectively. On average, females in the field laid 101.4 $\pm 8.0,107.8 \pm 14.8$ and $115.8 \pm 20.5$ eggs, respectively. The differences in realised fecundity between years were not statistically significant $(F=0.19, P=0.831)$. In contrast, the difference between potential and realised fecundity was highly significant within each year (2004: $F=11.74$, $P=0.001 ; 2005: F=46.46, P<0.001 ; 2006: F=33.18, P<0.001)$. Comparisons of potential and realised fecundity show that only $28.9 \%, 36.3 \%$ and $35.9 \%$ of available eggs were successfully laid in the field in 2004, 2005 and 2006, respectively. As shown in Tables 2-1, 2-2 and 2-3, this lost fertility accounts for $15.9-20.3 \%$ of generational mortality. While leek moth fecundity remained fairly constant during this three-year study, the variability in pre-imaginal mortality rates led to quite different outcomes in terms of population dynamics. In both 2004 and 2005, the third generation population declined $\left(R_{0}=0.185\right.$ and 0.502 , respectively) whereas it would have doubled in $2006\left(R_{0}\right.$ $=2.095)$.

\subsubsection{Key Factor Analysis}

The graphic approach of Varley and Gradwell (1960) did not prove effective for identifying key mortality factors. On the other hand, regressing $k$-values against $K$ gave strong evidence that mortality during neonate establishment $\left(k_{2}\right)$ and the combined larval instars $\left(k_{3}\right)$ are key elements driving population changes in this study system. These two factors showed significant correlation to total mortality and had the highest slopes (Table $2-4)$. To test whether the correlation between the combined larval $k$-values and $K$ was 
largely due to a particular instar, the five instars were re-analysed independently. This second analysis showed that the first instar was chiefly responsible $\left(k_{3(l)}=0.187\right)$, but as this regression coefficient is only half that for all larval instars combined, it is clearly not the only instar of influence.

\subsubsection{Field Exploration for Parasitoids}

At no point over two years of sampling were naturally occurring leek moth eggs discovered on leeks or onions, even though small numbers of adult moths were caught in pheromone traps at all sites. Furthermore, there was no evidence of any egg parasitism in 2004 or 2005 , despite having placed several thousand leek moth eggs on sentinel leeks in organic and integrated production Allium crops. In contrast, several hymenopteran parasitoids were reared from field-collected larvae and pupae, with the greatest numbers and diversity occurring in survey sites with high natural leek moth populations. Four species were reared from host larvae: Diadegma chrysostictos (Gmelin) (Ichneumonidae), D. fenestrale Holmgren (Ichneumonidae), Pnigalio soemius (Walker) (Eulophidae) and $P$. pectinicornis (Linnaeus) (Eulophidae). The pupae were parasitised by two pupal parasitoids: Diadromus pulchellus Wesmael (Ichneumonidae) and Itoplectis maculator (Fabricius) (Ichneumonidae).

The candidacy of these six parasitoid species for classical biological control was compared by reviewing host-records in the literature for each one to estimate its host specificity and the intimacy of its association with leek moth. This was an effective filter to identify the most suitable agent. Diadromus pulchellus was the only parasitoid suspected to be relatively host-specific. There are no known records of this parasitoid emerging from any hosts other than leek moth in the field, whereas the other five parasitoid species have been associated with between 29 and 124 host species (Yu et al., 2005). Moreover, D. pulchellus was the most abundant parasitoid on leek moth and it was the only species found attacking the pest in all of the three leek moth generations. Diadromus pulchellus was the sole species obtained from leek moth in the final generation. A final argument for the possible suitability of this parasitoid for biological control is that a congeneric species, Diadromus collaris (Gravenhorst) (Ichneumonidae), has been used extensively in biological control programmes worldwide against Plutella 
xylostella (Linnaeus) (Lepidoptera: Plutellidae), with proven efficacy (Sarfraz et al., 2005) and no evidence of non-target effects. Based on these observations, D. pulchellus was selected as the candidate agent with the greatest potential for classical biological control in Canada.

\subsection{Discussion}

Life tables have been heavily employed in insect ecology as they are effective tools for measuring inter- and intra-generational population change and for quantifying the impact of specific mortality factors (Harcourt, 1969; Bellows et al., 1992; Carey, 2001). This study compared stage-specific mortality rates of leek moth and thereby identified mortality 'of key stages that drive population change in central Europe. Variation in mortality values was greater among years than among sites, however the site-specific survivorship curves (Figure 2-1) are relatively consistent and there is a distinct pattern to the distribution of mortality across all pre-imaginal life stages (Figure 2-2). In his study of leek moth field mortality in England, Noyes (1974) observed a different mortality trend, with the bulk of generational mortality occurring in the final pre-imaginal stages. He recorded extremely low egg and larval mortality (3\% and 7\%, respectively), whereas the results here showed egg mortality rates between $49 \%$ and $72 \%$ and mortality for the entire larval period ranging from $60 \%$ to $85 \%$. Another discrepancy between these two studies is that while the current found increased survival of certain life stages within the exclusion cages, Noyes (1974) detected no cage treatment effect. Such incongruity between studies, or even between life tables within a single study, is not uncommon (e.g., Rausher, 1979; Kuhlmann et al., 1998; Furlong et al., 2004; Naranjo and Elsworth, 2005; Toepfer and Kuhlmann, 2006) and should perhaps be expected, given regional idiosyncrasies and environmental stochasticity (Cornell and Hawkins, 1995). Hence, to ensure robust mortality estimates, it is important to obtain life table data from multiple sites and generations (Morris, 1955). Thus, single-sample life tables should be interpreted with extreme caution. Even with adequate replication, there is no guarantee that inferences drawn from a particular life table would be applicable to insect systems several years later or outside the original study region. 
Although it is well-known that newly hatched larvae of both endophytic and exophytic species are generally very vulnerable to numerous mortality factors (Zalucki et al., 2002), relatively few studies have closely assessed the mortality occurring between egg hatch and the establishment of a feeding site by neonate larvae. Published examples do exist (e.g., Lee, 1988; Furlong et al., 2004); however, deaths in this phase are most often included in a total value for first instar mortality. For tissue-mining species, like leek moth, whose eggs are laid on the surface of the plant, the time between hatch and plant penetration may be one of the few, or only, moments when the larvae are exposed on the exterior surface of the host plant. While there are some exceptions, for example when neonate larvae burrow into the plant directly below the egg (Martin, 1956; Cappuccino, 1991), most species have a pre-feeding wandering phase following egg hatch (Zalucki et al. 2002). This post-hatch roaming behaviour is common in leek moth (Noyes, 1974); however, it was not previously known how the short but hazardous period affects leek moth survival. This study convincingly demonstrates the importance of this very brief juncture in the leek moth life cycle. Neonate mortality played a main role in generational mortality in 2004 and 2005. Then, in 2006 when this mortality value dropped by more than half from previous estimates, the overall pre-imaginal survivorship soared. Eggs laid in 2006 were ten times more likely to survive to the adult stage than those laid in 2004. Considering that neonates may take approximately two hours to penetrate a leek leaf, this is a remarkably short period for the consequences it can have on population dynamics. This effect was detected in the key factor analysis, which ranked neonate mortality as the primary key factor influencing population change.

When the leek moth larval instars were considered separately, no specific instar was a key contributor to total mortality. However, total larval mortality was one of the major mortality factors during leek moth development. This agrees with the outcome of the key factor analysis. In a similar example involving an endemic New Zealand butterfly, larval mortality at first appears relatively insignificant when the instars are considered independently (Barron et al., 2003). Nonetheless, once the five instars were pooled, larvae accounted for nearly half of the generational mortality.

The high overall larval mortality was contrary to expectations. It was anticipated that the larval stage would experience the greatest survival since the larvae feed within a 
structural refuge, whereas the eggs and pupae are fully exposed on the external plant surface. Meta-analyses comparing survivorship in species with different feeding habits have shown that exophytic species tend to experience greater pre-adult mortality than endophytic species (Price, 1975; Cornell and Hawkins, 1995). It is possible that this across-species generalisation is not a good predictor of within-species mortality distribution when certain life stages are endophytic and others are exophytic. Interestingly, there are no equivalent syntheses that attempt to elucidate patterns of stagespecific mortality rates within single species that have variable degrees of concealment during their pre-imaginal development. In many herbivorous insect species, the level of concealment within host plant tissues or surrounding substrates changes as individuals progress from one life stage to the next. As a case in point, leek moth would fall into the endophyte group under the traditional system of categorisation because all larval instars are leaf miners. The problem with this classification is immediately apparent as immature leek moths are actually on the exterior of their host plant during the egg, neonate and pupal phases, all of which contribute significantly to generational mortality. Particularly for species that suffer high levels of egg and pupal mortality, it is likely inappropriate to class them as strictly endophytic or exophytic based on the degree of concealment of the larval stage.

Kyi et al. (1991) remarked that life table studies often fail to identify causes of observed mortality. Over the last two decades, life tables have continued to play a key role in insect ecology and many of these studies clearly distinguish between specific mortality factors (e.g., Floater and Zalucki, 1999; Barron et al., 2003; Pereira et al., 2007; Girardoz et al., 2007) owing to the development of improved experimental and sampling techniques (Luck et al. 1988; Bellows et al. 1992). Nonetheless, unassigned mortality is still commonplace in even the most recent insect life tables, suggesting that significant hurdles still remain in assessing field mortality. Based on 124 herbivore life tables, Cornell et al. (1998) calculated an average of approximately $20 \%$ mortality due to unknown factors over all stages. Although the current study obtained little information on specific mortality factors for each life stage, the comparison of mortality rates on caged and uncaged leeks serves as a first step in quantifying the relative contribution of natural enemies. As illustrated in Figure 2-2, there was substantial mortality even in the 
exclusion treatments, particularly during the egg and neonate stages where mean mortality for each was approximately $50 \%$. For eclosing first instar larvae, plant factors may have played a key role, while inclement weather was very likely a cause of mortality among both eggs and neonate larvae. Simple observations of establishing neonates have shown that, while on the leaf surface, these tiny larvae are easily dislodged suggesting that light wind or rain would be lethal if it coincided with egg hatch. The survival probability for newly hatched larvae that become separated from their host plants is poorly understood, but is generally assumed to be very low (Zalucki, 2002). Dislodgement can even be costly for larvae that remain on the plant because they often tumble into the leaf axils where rainwater collects. While late instar larvae have been seen to pull themselves out of these pools, neonate larvae are especially vulnerable to drowning.

The apparent lack of parasitism in this life table study matches Noyes' (1974) observations but was not expected as there are a number of published accounts of leek moth parasitoids in continental Europe. These include both larval and pupal parasitoids (Frediani, 1954; Labeyrie, 1966; Plaskota and Dawbrowski, 1986), whereas there are no records of egg parasitism. Previous descriptions of parasitism have typically come from locations with sustained leek moth outbreaks, whereas natural populations in Switzerland during this study were generally so low that it was often not possible to detect plant damage, much less to sample naturally occurring leek moths. This low host abundance may have led to such significant numerical reductions of parasitoids that they were difficult to detect using small experimental populations of leek moth placed within relatively large commercial fields. Furthermore, nearly all of the leek moth parasitoid species that were collected through random field sampling were polyphagous. Generalist foragers have been shown to undergo shifts in host preference in response to changes in the relative abundance of host species (Cornell and Pimentel, 1978; Duan et al., 2000). The artificial infestations may have been too small to stimulate generalist parasitoids to actively search for leek moth hosts.

Alternatively, low parasitism in the life table study may simply have reflected severely reduced parasitism levels at the end of the growing season. Continuous field samples of larvae and pupae from one heavily infested site in 2005 showed a diminishing 
parasitoid impact from mid- to late-summer, such that only D. pulchellus was reared from leek moth, but at a lower frequency than in the previous generations. Consequently, a similar life table study conducted earlier in the summer might have revealed a much stronger contribution of parasitoids to leek moth mortality. Shifts in parasitoid community structure and impact across generations have been described for leek moth by Plaskota and Dabrowski (1986); however, these authors reported an increase in parasitoid-induced mortality with each subsequent generation. This discrepancy may be partly explained by differences in parasitoid species composition. Once again, this illustrates the need for region-specific life tables.

Owing to the lack of parasitoids emerging from leek moth in the life table study, predation is believed to have been the primary form of natural enemy-induced mortality. The greatest and most consistent differences in mortality between caged and exposed leeks were observed for the pupae, suggesting that this stage is particularly vulnerable to predators. Indeed, leek moth pupae tend to be very exposed on the leaf surface and mirid and anthocorid bugs and chrysopid larvae were occasionally seen feeding on them during this study. There were no apparent signs of pathogen-related mortality in any leek moth life stages and this is perhaps not surprising given that diseases tend not to be important factors in leaf-mining species (Auerbach et al., 1995).

In conclusion, this study found a propensity for higher leek moth mortality in the exposed egg and pupal stages compared to the individual leaf-mining larval stages. However, when instars were pooled, larval mortality nearly always exceeded that of eggs and pupae. This life table study used an approach that provided the resolution necessary to uncouple mortality associated with neonate establishment from that which occurs after successful establishment on the host plant. It revealed that an important fraction of generational mortality occurs in the brief period of exposure following egg hatch. Now that this critical stage has been identified, it would be possible to conduct experiments that focus on the specific factors causing the high neonate mortality. The study also showed that while natural enemies appeared to have only a transient effect on leek moth egg and larval survival, they were a consistent and significant factor in pupal mortality. Thus, in terms of biological control as a management option in Canada, the pupal stage could be a vulnerable target with the greatest likelihood of successful impact. A logical 
follow-up to this study would be a similar evaluation of mortality distribution over the pre-imaginal stages of leek moth in its introduced range. This could facilitate a better understanding of why Canadian leek moth populations tend to reach higher densities than European populations. Finally, based on field collections and a thorough literature review, the parasitoid $D$. pulchellus was identified as the most promising candidate for introduction in North America. The following chapters describe the evaluation of this agent for classical biological control of leek moth. 
Table 2-1. Life table for the third generation of leek moth, Acrolepiopsis assectella. Data are pooled from three sites in Switzerland in 2004. $l_{x}=$ number entering stage; $d_{x}=$ number dying in stage; $q_{x}=$ apparent mortality; $m_{x}=$ marginal mortality.

\begin{tabular}{llrrrrrr}
\hline Life stage & Mortality factor & \multicolumn{1}{c}{$l_{x}$} & \multicolumn{1}{c}{$d_{x}$} & $100 q_{x}$ & $m_{x}$ & $k$-value & $\begin{array}{c}\text { \% generational } \\
\text { mortality }\end{array}$ \\
\hline Eggs & Unknown & $\mathbf{1 0 0 0 . 0}$ & 720.0 & 72.0 & 72.0 & 0.553 & 16.9 \\
Neonate & Unknown & 280.0 & 230.2 & 82.2 & 82.2 & 0.750 & 22.9 \\
L1 & Unknown & 49.8 & 23.0 & 46.2 & 46.2 & 0.269 & 8.2 \\
L2 & Unknown & 26.8 & 10.8 & 40.0 & 40.0 & 0.222 & 6.8 \\
& Parasitoid & 16.1 & 0.1 & 0.4 & 0.5 & 0.002 & 0.1 \\
L3 & Unknown & 16.0 & 4.4 & 27.2 & 27.2 & 0.138 & 4.2 \\
& Parasitoid & 11.7 & 0.1 & 0.6 & 0.7 & 0.003 & 0.1 \\
L4 & Unknown & 11.6 & 2.0 & 17.0 & 17.0 & 0.081 & 2.5 \\
L5 & Unknown & 9.6 & 2.1 & 21.9 & 21.9 & 0.107 & 3.3 \\
Pupae & Unknown & 7.5 & 3.9 & 51.3 & 51.3 & 0.312 & 9.5 \\
Adults emerged & Sex ratio & 3.7 & 1.8 & 50.0 & 50.0 & 0.301 & 9.2 \\
Adult females & & 1.8 & & & & & 16.5 \\
Potential progeny & Lost fertility & 640.6 & 455.7 & 71.1 & 71.1 & 0.540 & \\
Realised progeny & & 184.9 & & & & & \\
\hline Total mortality $=99.6 \%$ & & & & & & &
\end{tabular}

* Potential progeny $=$ (\# of surviving females) $\times$ (mean potential fecundity from 2004).

* Realised progeny $=$ (\# of surviving females) $\times$ (mean realised fecundity from 2004). 
Table 2-2. Life table for the third generation of leek moth, Acrolepiopsis assectella. Data are pooled from three sites in Switzerland in 2005. $l_{x}=$ number entering stage; $d_{x}=$ number dying in stage; $q_{x}=$ apparent mortality; $m_{x}=$ marginal mortality.

\begin{tabular}{lcrrrrrr}
\hline Life stage & Mortality factor & \multicolumn{1}{c}{$l_{x}$} & \multicolumn{1}{c}{$d_{x}$} & $100 q_{x}$ & $m_{x}$ & $k$-value & $\begin{array}{c}\text { \% generational } \\
\text { mortality }\end{array}$ \\
\hline Eggs & Unknown & 1000.0 & 492.0 & 49.2 & 49.2 & 0.294 & 10.6 \\
Neonate & Unknown & 508.0 & 423.2 & 83.3 & 83.3 & 0.777 & 28.0 \\
L1 & Unknown & 84.8 & 25.3 & 29.8 & 29.8 & 0.154 & 5.5 \\
L2 & Unknown & 59.6 & 15.7 & 26.4 & 26.4 & 0.133 & 4.8 \\
L3 & Unknown & 43.8 & 9.0 & 20.6 & 20.6 & 0.100 & 3.6 \\
L4 & Unknown & 34.8 & 2.8 & 8.1 & 8.1 & 0.037 & 1.3 \\
L5 & Unknown & 32.0 & 5.7 & 17.8 & 17.8 & 0.085 & 3.1 \\
Pupae & Unknown & 26.3 & 17.0 & 64.6 & 64.6 & 0.451 & 16.3 \\
Adults emerged & Sex ratio & 9.3 & 4.7 & 50.0 & 50.0 & 0.301 & 10.9 \\
Adult females & & 4.7 & & & & & 15.9 \\
Potential progeny* & Lost fertility & 1382.1 & 880.4 & 63.7 & 63.7 & 0.440 & \\
Realised progeny** & 501.6 & & & & & \\
\hline Total mortality $=99.1 \%$ & & & & & & & \\
Net reproductive rate $\left(R_{0}\right)=0.502$ & & & & & & \\
\hline
\end{tabular}

* Potential progeny $=$ (\# of surviving females) $\times$ (mean potential fecundity from 2005).

** Realised progeny $=$ (\# of surviving females) $\times$ (mean realised fecundity from 2005). 
Table 2-3. Life table for the third generation of leek moth, Acrolepiopsis assectella. Data are pooled from three sites in Switzerland in 2006. $l_{x}=$ number entering stage; $d_{x}=$ number dying in stage; $q_{x}=$ apparent mortality; $m_{x}=$ marginal mortality.

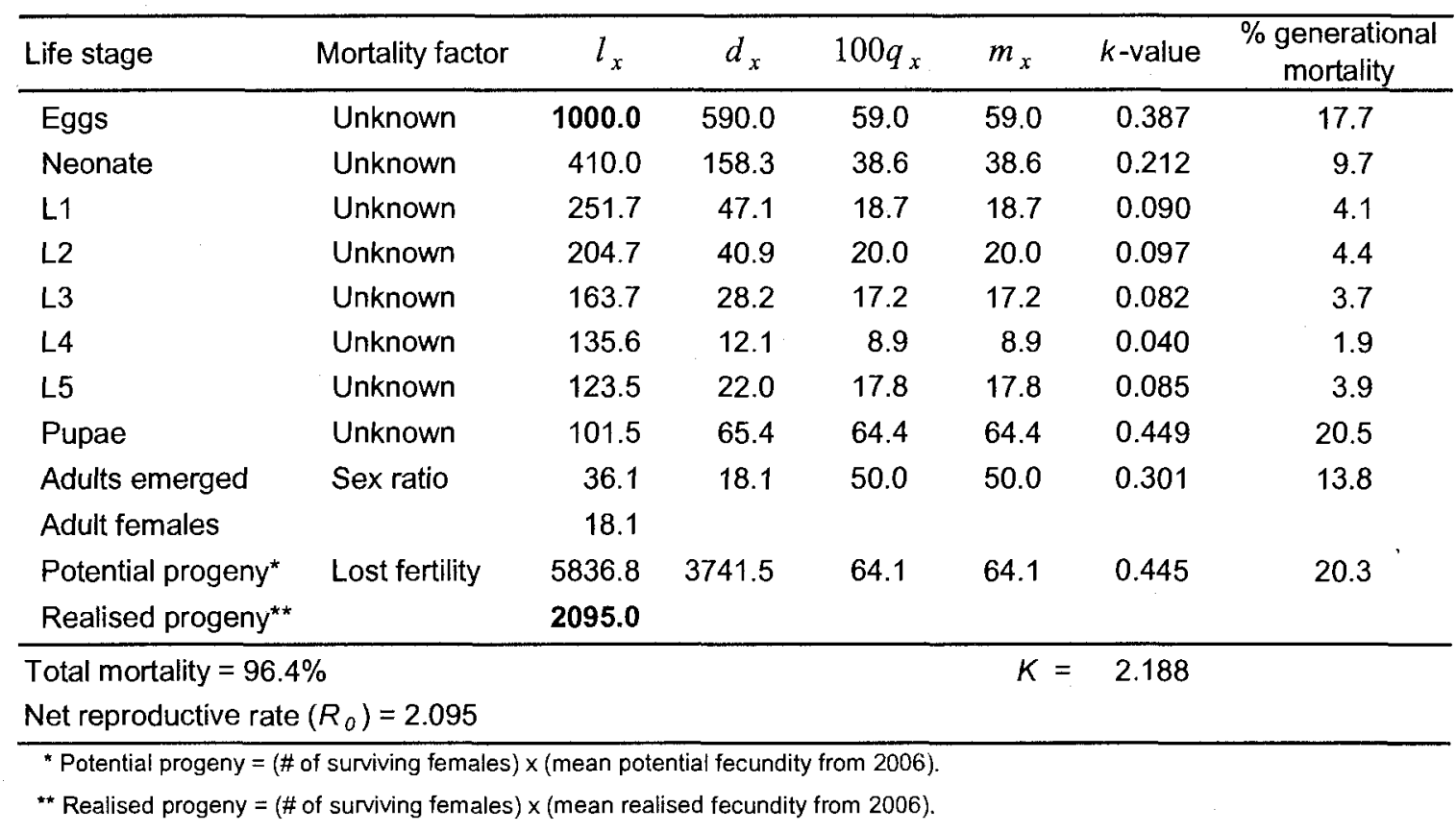


Table 2-4. Key factor analysis of mortality factors affecting Acrolepiopsis assectella populations at three sites between 2004 and 2006 using regression techniques as described by Podoler \& Rogers (1975). $k_{1}=$ egg; $k_{2}=$ establishing neonates; $k_{3}=$ combined larval instars; $k_{3(I)}=1^{\text {st }}$ instar; $k_{3(2)}=2^{\text {nd }}$ instar; $k_{3(3)}=3^{\text {rd }}$ instar; $k_{3(4)}=4^{\text {th }}$ instar; $k_{3(5)}=5^{\text {th }}$ instar; $k_{4}=$ pupa; $k_{5}=$ lost fertility.

\begin{tabular}{ccc}
\hline $\begin{array}{c}\text { Mortality factor } \\
(k)\end{array}$ & $\begin{array}{c}\text { Regression } \\
\text { coefficients }(b)\end{array}$ & $\begin{array}{c}\text { Determination } \\
\text { coefficients }\left(r^{2}\right)\end{array}$ \\
\hline$k_{I}$ & 0.061 & 0.024 \\
$k_{2}$ & 0.545 & 0.653 \\
$k_{3}$ & 0.364 & 0.794 \\
$k_{3(1)}$ & 0.187 & 0.867 \\
$k_{3(2)}$ & 0.083 & 0.381 \\
$k_{3(3)}$ & 0.042 & 0.182 \\
$k_{3(4)}$ & 0.031 & 0.193 \\
$k_{3(5)}$ & 0.022 & 0.066 \\
$k_{4}$ & -0.025 & 0.001 \\
$k_{5}$ & 0.055 & 0.048 \\
\hline
\end{tabular}




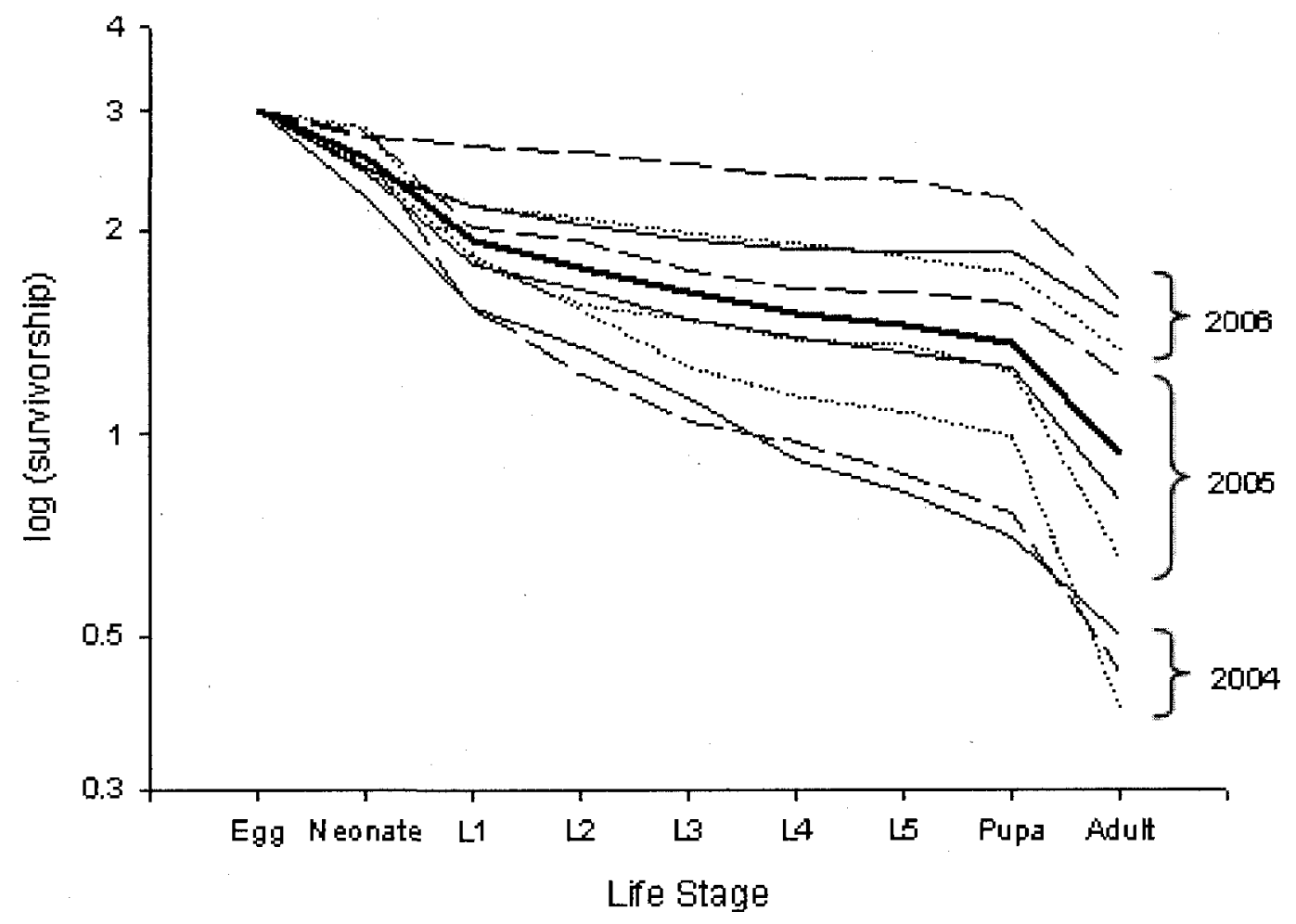

Figure 2-1. Survivorship curves for immature Acrolepiopsis assectella at three commercial leek production sites from 2004 to 2006 (thin solid lines = Site A; dashed lines $=$ Site $\mathrm{B}$; dotted lines $=$ Site $\mathrm{C}$; bold line $=$ mean survivorship) . 

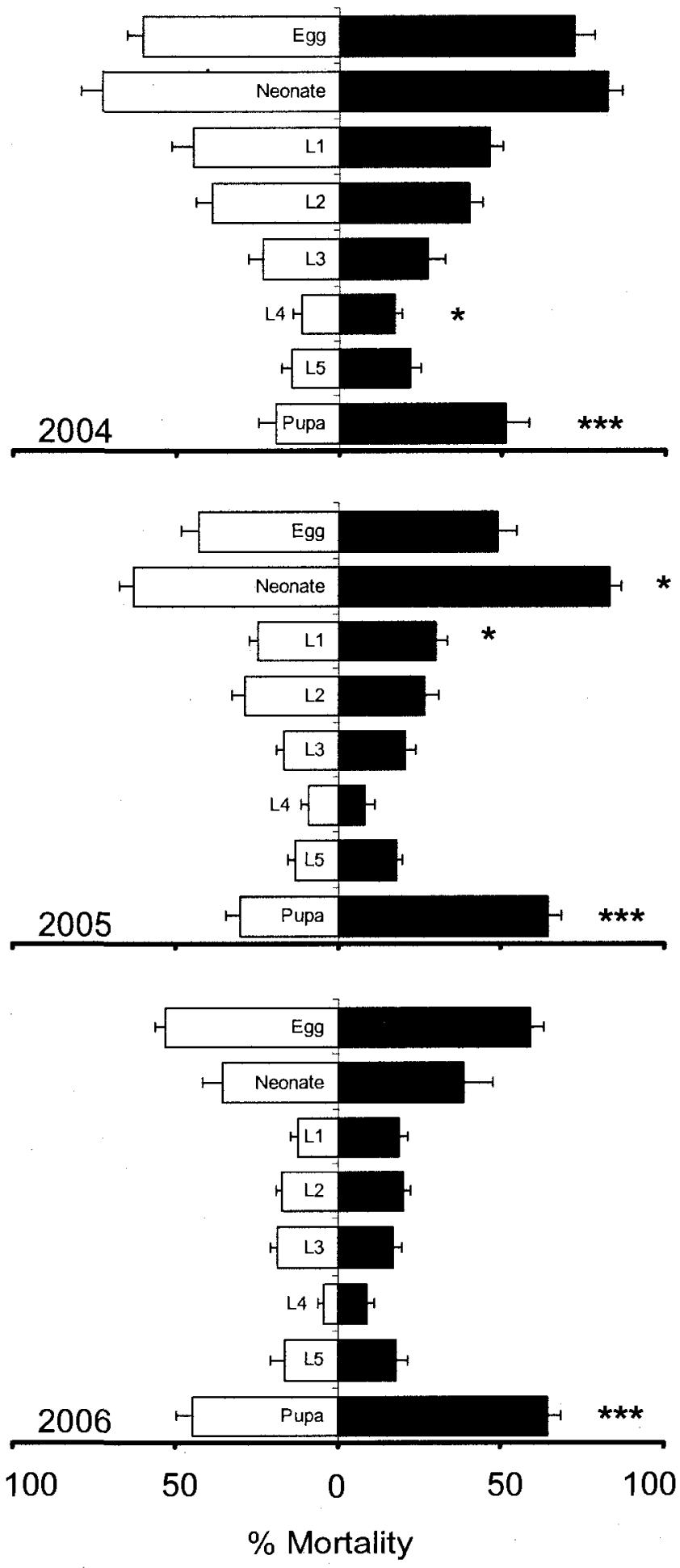

Figure 2-2. Comparison of stage-specific Acrolepiopsis assectella mortality between caged (white bars) and uncaged (black bars) infested leeks. Error bars show 1 S.E. Asterisks denote life stages where the exclusion treatment significantly affected mortality (Wilcoxon paired-sample test: $* P<0.05 ; * * * P<0.001$ ). 


\section{Cold Tolerance and Overwintering Strategy of Diadromus pulchellus}

\subsection{Introduction}

Measuring success in classical biological control is an important, but difficult, process; therefore, several reviews discuss the frequency with which such endeavours have achieved satisfactory suppression of pests (Hall and Ehler, 1979; Greathead, 1986; Hagvar, 1991; Greathead and Greathead, 1992; Gurr et al., 2000). One frequent observation is that a substantial proportion of release programmes result in little or no success and in many cases this appears to have been due to the failure of natural enemies to establish. Since the intention of classical biological control is to introduce a selfperpetuating mortality factor to reduce pest numbers, the permanent establishment of the agent is an essential element of the process. When released agents fail to establish in their new environments, it can be difficult to identify the cause of failure and this may partly explain the paucity of published explanations for unsuccessful releases. Stiling (1993) listed several factors that have previously been blamed for preventing biological control agents to establish. These include issues such as poor climate matching, lack of synchronisation between natural enemy and pest, competition with other natural enemies and Allee effects resulting from inadequate numbers of individuals released. Among these inhibitory factors, climate matching is considered to be one of the greatest impediments to classical biological control (DeBach and Bartlett, 1964; Stiling, 1993).

Much research has been dedicated to identifying the nature of insect overwintering strategies and the mechanisms controlling them. Parasitoids are similar to other insects in that the overwintering stage and the cues that induce an overwintering state are highly variable (Tauber et al., 1986). Particularly for northern latitudes, knowledge of an insect's cold tolerance and overwintering biology is central to assessing its potential to establish and become a fixed component in a new environment (McDonald et al., 2000). If too few individuals survive the winter or if cool temperatures preclude successful oviposition and development, then establishment of a permanent population will be unlikely (Leather et al., 1993). A number of recent studies have examined the cold hardiness of proposed and already-used biological control agents for greenhouse pest 
management in the UK (e.g., Hart et al., 2002; Hatherly et al., 2004; Larentzaki et al., 2007). The motivation behind these studies was to flag and perhaps rule out those agents that demonstrated a strong potential to establish permanently if they would escape from their greenhouse environment. These authors employed a suite of experiments to measure species-specific attributes such as thermal budget, lower developmental threshold, supercooling point, lower lethal temperature and lethal time at a constant low temperature. The cold tolerance data obtained from these types of controlled laboratory tests may indeed reflect a species' capacity to withstand winter conditions in the area of introduction (Campbell et al., 1974; Hathery et al., 2005) and could prove useful in other biological control programmes.

Diadromus pulchellus Wesmael (Hymenoptera: Ichneumonidae), a pupal parasitoid of leek moth, Acrolepiopsis assectella (Zeller) (Lepidoptera: Acrolepiidae), is under consideration for introduction into Canada to reduce leek moth populations. Despite many years of research on $D$. pulchellus, its mode of overwintering has not been identified. Clarifying this parasitoid's overwintering strategy and cold hardiness is considered important for two reasons. First, it will provide an indication of the agent's ability to overwinter in Canada. Second, it may help to determine whether D. pulchellus has alternate hosts. Because leek moth and $D$. pulchellus adults emerge from pupae at the end of the third and final leek moth generation, there should be no leek moth pupae available in the field as overwintering hosts for parasitoids emerging at the end of the season. Thus, if $D$. pulchellus parasitoids require hosts in which to produce an overwintering generation, they would have to find an alternative host species. However, if $D$. pulchellus overwinters as an adult, no overwintering hosts would be necessary.

The objectives of this study were to elucidate the overwintering strategy and cold hardiness of D. pulchellus and the likelihood of its permanent establishment in Canada. However, due to quarantine restrictions on the candidate agent in North America, it was not possible to directly assess winter survival under true Canadian conditions. Therefore, a series of experiments were conducted under laboratory conditions and in the field in the agent's area of origin. All growth chamber and outdoor experiments were conducted at the CABI Europe-Switzerland Centre. The experiments specifically measured this agent's (1) lower and upper developmental thresholds, (2) thermal budget and (3) pre-imaginal 
and adult survival at sustained cold temperatures. Furthermore, both immature and adult parasitoids were exposed to winter conditions in central Europe to assess survival. Finally, oviposition tests were run to determine whether females could still reproduce following development at or adult exposure to cold temperatures.

\subsection{Methods}

\subsubsection{Insect Rearing}

A laboratory colony of $D$. pulchellus was established with parasitoids reared from leek moth pupae collected in organic and integrated production leek fields in west-central Switzerland in 2005. The parasitoids were reared continuously on leek moth in the laboratory with field-collected specimens being added to the culture each summer. Emerging parasitoids were stored in mesh-walled, $30 \mathrm{~cm}^{3}$ plastic cages (BugDorm-1; BioQuip $^{\circledR}$, Rancho Domingo, CA, USA) and provided with a $20 \%$ sucrose solution. Leek moth hosts were mass-produced on potted leeks in the laboratory $\left(22 \pm 2^{\circ} \mathrm{C}, 16 \mathrm{~L}: 8 \mathrm{D}\right)$. Newly formed pupae were offered en-masse to $D$. pulchellus in the BugDorms for oviposition to maintain the parasitoid culture. Following 24 hours of exposure, the parasitised pupae were stored in Petri dishes at $22 \pm 2^{\circ} \mathrm{C}, 16 \mathrm{~L}: 8 \mathrm{D}$ until emergence of adults.

\subsubsection{Developmental Thresholds and Thermal Budget}

The emergence rate and development time of immature D. pulchellus (egg to adult emergence) in leek moth hosts was measured at temperatures ranging between 7.5 and $35^{\circ} \mathrm{C}$ with $2.5^{\circ} \mathrm{C}$ increments ( $=12$ temperature treatments) under a $16 \mathrm{~L}: 8 \mathrm{D}$ light regime. Rearing temperatures fluctuated around the target by $\pm 1{ }^{\circ} \mathrm{C}$. Pupae were placed into parasitoid cages for 24 hours at $22 \pm 2^{\circ} \mathrm{C}$ to facilitate parasitism. The hosts were then divided into large cohorts of approximately 150 pupae and stored in Petri dishes that were allocated to different experimental temperatures. It should be noted here that not all pupae exposed to parasitoids would have been parasitised, therefore emergence rates reflect both the initial degree of parasitism as well as progeny survival. Parasitism rates may have differed depending on the day of parasitism or on which parasitoid cages had been used for oviposition. To minimise the effect of these potentially confounding 
factors, the pupae were always mixed after removal from parasitoid cages and then randomly allocated to Petri dishes for the temperature treatments. The Petri dishes in all temperature treatments were checked daily to record emergence of males and females and trials were stopped seven days after the last adult emerged. All temperature treatments were replicated five times while additional replicates were run for temperatures suspected to be around the upper and lower developmental threshold, where survival was expected to be weak. The effect of rearing temperature on $D$. pulchellus emergence rate was analysed with ANOVA. All statistical analyses were conducted using SPSS version 14.0 (SPSS Inc, Chicago, IL).

As there were no significant differences between male and female development times at specific temperatures, the sexes were pooled for analysis. A single mean value for development time was calculated for each Petri dish (cohort) of each treatment. Parasitoid development rates were then calculated by taking the reciprocal of development time (1/days until emergence). The relationship between rearing temperature and development rate is linear over the middle range of temperatures but becomes curvilinear at the lower and higher extremes (Campbell et al., 1974). The lower developmental threshold can be estimated through extrapolation of the linear model (i.e., extending the line until it cuts the x-axis) (Campbell et al., 1974). To calculate the developmental threshold for $D$. pulchellus, a simple linear regression was performed using data from the range of $15-27.5^{\circ} \mathrm{C}$. The thermal budget (day-degree requirement) was then calculated by taking the reciprocal of the slope (Campbell et al., 1974).

In addition to monitoring emergence rates and development times, the effect of developmental temperature on reproductive capacity was assessed. A sub-sample of three females and three males emerging from each Petri dish was set up in a clean Petri dish with a cotton wick containing $20 \%$ sucrose solution. Following a three-day preoviposition period, the parasitoids were provided with four fresh leek moth hosts per day over three days ( $=12$ hosts). The host pupae were removed after a 24-hour exposure and stored in marked Petri dishes until emergence of parasitoid offspring to assess the success of parasitism. 


\subsubsection{Effect of Variable Durations at Cool Temperatures on Pre-Imaginal Survival}

The impact of above-freezing cool temperatures on immature $D$. pulchellus survival was studied by subjecting recently parasitised leek moth pupae to different cold temperatures for varying lengths of time and assessing the survival of parasitoid progeny (expressed as parasitoid emergence rate). To prepare a single treatment replicate, approximately 1301 -day-old leek moth pupae were placed into a cage with several $D$. pulchellus females to facilitate parasitism of as many pupae as possible. These pupae were then stored together in a Petri dish for 3 days at $22 \pm 1^{\circ} \mathrm{C}$. On day 3 , the pupae were randomly divided into groups of 15 and transferred to small, tight-sealing Petri dishes so that there were 7 groups of 15 pupae. Visibly unhealthy or dead pupae were removed at this stage. All 7 of these groups of pupae from one original batch of 130 were randomly assigned to a temperature treatment $\left(3,8\right.$ or $\left.12 \pm 1{ }^{\circ} \mathrm{C}\right)$. Each dish was then allocated to one of the following treatment durations: 0 weeks (control, maintained at $22^{\circ} \mathrm{C}$ ), 1 week, 2 weeks, 3 weeks, 4 weeks, 7 weeks or 10 weeks. For this experiment, the pupae were always stored in constant darkness, regardless of temperature, since the absence of a photophase had previously been shown not to affect $D$. pulchellus survival (t-test: $t=$ $0.256, P=0.802$ ) or development time (t-test: $t=0.759, P=0.463$ ). Following a cold temperature exposure, for example 2 weeks, the Petri dish designated for the 2-week treatment was transferred to the $22 \pm 1^{\circ} \mathrm{C}$ chamber and stored there until adult emergence was complete. All dishes were checked daily to record parasitoid emergence times. A total of 18 sets of 130 parasitised pupae were prepared to obtain six replicates of each of the three temperature treatments. Emergence rates were calculated for each dish under different cold exposure durations. Arcsine square-root transformation failed to normalise the data from the $3^{\circ} \mathrm{C}$ treatment, therefore the Kruskal-Wallis One-Way ANOVA on Ranks was used to compare emergence rates for different exposure durations within each temperature treatment.

\subsubsection{Pre-Imaginal Development under Autumn Conditions}

Newly parasitised pupae were subjected to outdoor conditions in late September and early October to simulate a scenario in which leek moth hosts become parasitised at the end of the growing season. This experiment was designed to test how ambient autumn 
conditions affect parasitoid survival and also whether the significantly decreasing day length and temperatures could induce a pre-imaginal overwintering state. From 24 September to 16 October, large cohorts of 1-day-old leek moth pupae were placed in $D$. pulchellus cages for parasitism. After 24 hours, these hosts were removed from the parasitoid cages and divided into two groups (100-250 pupae), each placed in a Petri dish and allocated to either the $20^{\circ} \mathrm{C} 16 \mathrm{~L}: 8 \mathrm{D}$ control or the outdoor treatment. For the outdoor treatment, Petri dishes were placed under a shaded and open-ended poly-ethylene tunnel outside the research centre. To facilitate full exposure of the pupae to ambient conditions, the dish lids were removed and a gauze fabric was fastened over the dishes with an elastic band. There were a total of 31 paired Petri dish cohorts in the outdoor treatment and the $20^{\circ} \mathrm{C}$ control. These dishes were checked daily throughout the entire autumn and winter to measure emergence success. Student's t-test was used to compare arcsine transformed emergence rates between the outdoor treatment and the indoor control. Illustrated data are untransformed.

A temperature data logger (HOBO ${ }^{\circledR}$ H8 Pro Series; ONSET, Pocasset, MA, USA) was placed among the Petri dishes under the poly-ethylene tunnel to measure the temperature hourly over the course of the entire experiment (24 September, 2007 to 1 March, 2008). Temperatures recorded inside the poly-ethylene tunnel where parasitised pupae were stored for the winter indicate that the days became substantially cooler by mid-October. The nighttime temperatures dropped below freezing on several occasions between November and February and, in a few rare cases during the coldest periods, the daytime maximum temperatures remained below the freezing point (Figure 3-1). The coldest temperature recorded was $-10.5^{\circ} \mathrm{C}$, however, freezing temperatures did not often fall below $-5^{\circ} \mathrm{C}$. At the upper end of the temperature spectrum, maximum temperatures exceeded $15^{\circ} \mathrm{C}$ on a number of occasions, but not in November, December or early January.

Finally, the ability of parasitoids to mate and oviposit following their period of winter development was tested. Upon eclosion, three females were placed into Petri dishes with three males and a cotton bud soaked with $20 \%$ sucrose solution. On day 3 , the females began receiving fresh hosts ( 3 per day for 7-10 days). These host pupae were removed after a 24-hour exposure and stored in labelled Petri dishes to record the 
emergence of parasitoid progeny. The reproductive success of outdoor-reared females is compared with that of females from the $20^{\circ} \mathrm{C}$ control.

\subsubsection{Adult Overwintering Success}

The adults of D. pulchellus are known to be long-lived and preliminary observations of caged individuals had indicated that they are tolerant of extended periods of cold temperatures. Adult overwintering ability was assessed with an experiment in which adults were stored at different temperature regimes for a period of three months (late November to late February). Hundreds of newly emerged male and female parasitoids were stored in BugDorm cages with a $20 \%$ sucrose solution at $12^{\circ} \mathrm{C}$ for one week. The adults were then divided into several same-sex groups of 20 individuals and placed into 1L plastic cylinder cages. Each cage had two small Petri dishes with loosely packed soil and two over-turned egg carton "cups", and was then filled with shredded and crumpled paper. Pure water was provided through two cotton wicks at the base of the cage. Once the parasitoids were moved to these smaller cages, they were progressively chilled to their treatment temperatures. They were first placed back at $12^{\circ} \mathrm{C}$ for two hours, then at $6^{\circ} \mathrm{C}$ for approximately 20 hours. The next day, the cylinder cages were then allocated to 1 of 4 overwintering treatments: (1) constant $-1^{\circ} \mathrm{C}$; (2) constant $1^{\circ} \mathrm{C}$; (3) constant $3{ }^{\circ} \mathrm{C}$ for four weeks, then constant $1{ }^{\circ} \mathrm{C}$ for two weeks and then constant $6^{\circ} \mathrm{C}$ for the final six weeks (hereafter called the $3,1,6^{\circ} \mathrm{C}$ treatment); (4) outdoor exposure under a shaded and open-ended poly-ethylene tunnel, with natural daily fluctuations. A total of 5 female cages and 5 male cages were allocated to each treatment. The water for each cage was re-filled throughout the winter as needed.

On 22 February, all cages were brought to $20^{\circ} \mathrm{C}$. Survival within each cage was measured the following day and the effect of overwintering treatment was assessed using a one-way ANOVA. When possible, a sub-sample of three surviving females from each cage was transferred to a Petri dish with $20 \%$ sucrose solution and provided with 3 fresh leek moth pupae each day for 6 days. No males were placed in the Petri dishes with the females since these females were likely to have been mated during storage prior to the overwintering experience. The host pupae provided to parasitoids were stored in marked Petri dishes and observed to record offspring emergence. 


\subsubsection{Adult Freeze Tolerance (LTime50)}

If $D$. pulchellus overwinters in the adult stage, as suspected, then adults should demonstrate some degree of tolerance to freezing temperatures, which occur in central Europe and, to a greater extent, in Canada. Winter air temperatures in Allium producing areas of eastern Ontario and western Quebec frequently drop to, and rest below, $-20^{\circ} \mathrm{C}$, however, daily averages in the coldest months are typically not much colder than $-10^{\circ} \mathrm{C}$. Furthermore, many overwintering insects are known to benefit from the insulating effect caused by a layer of snow on the ground (e.g., Shorthouse et al., 1980). Lethal time $\left(\right.$ LTime $\left._{50}\right)$ trials were conducted to examine survival of $D$. pulchellus adults under varying durations of exposure to freezing temperatures that are likely to be experienced in the intended release areas in Canada. The aim was to identify exposure times required to kill $50 \%$ of males and females exposed to either $-12^{\circ} \mathrm{C}$ (simulating winter temperatures without an insulating snow cover) or $-4^{\circ} \mathrm{C}$ (simulating winter temperatures under snowpack). Two sources of parasitoids were used for both temperature treatments. The first source was the regular $D$. pulchellus culture maintained under summer conditions $(22 \pm$ $\left.2^{\circ} \mathrm{C}, 16 \mathrm{~L}: 8 \mathrm{D}\right)$. Thus, these parasitoids experienced no pre-conditioning during preimaginal development. The second source was the previously-described outdoor exposure experiment (section 3.2.4) where parasitised pupae had been stored in a poly-ethylene tunnel immediately after oviposition by parasitoids in late September. Due to significantly lower emergence of parasitoids in the outdoor treatment, there were fewer adults available for freeze tolerance tests and therefore some exposure durations were only replicated once.

Newly emerged parasitoids from both summer and autumn rearing conditions were put into separate BugDorm cages with $20 \%$ sucrose solution and held at $12^{\circ} \mathrm{C}$ for $7-10$ days. Following this preliminary cold-hardening phase, the adults were divided into same-sex groups of 10 individuals and placed into Petri dishes with a cotton wick containing $20 \%$ sucrose solution. These dishes were placed back into $12^{\circ} \mathrm{C}$ for a further 4 days. Prior to subjecting the parasitoids to freezing temperatures, the sucrose wicks were removed from each Petri dish to reduce the risk of ice nucleation on the external body 
surfaces, which can affect the rate of freezing and survival in insects at low temperatures (Salt, 1963; Duman, 2001).

For the $-12^{\circ} \mathrm{C}$ treatment, the temperature was gradually reduced to the target temperature in 6-degree intervals. Parasitoids were held at $6^{\circ} \mathrm{C}$ for 1 hour, $0^{\circ} \mathrm{C}$ for 1 hour, $-6^{\circ} \mathrm{C}$ for 1 hour and finally $-12^{\circ} \mathrm{C}$. This gives a rate of temperature change of $1{ }^{\circ} \mathrm{C} / 10$ minutes or $0.1^{\circ} \mathrm{C} /$ minute, which is 10 times slower than the $1^{\circ} \mathrm{C} /$ minute system used in many studies (Leather et al. 1993). Once at $-12^{\circ} \mathrm{C}$, this temperature was held constant for the duration of the experiment (8L:16D). Each day for 7 days, a sample of dishes was removed from $-12^{\circ} \mathrm{C}$ and warmed gradually to $20^{\circ} \mathrm{C}$. After removal from the freeze treatment, the parasitoids were given $20 \%$ sucrose solution in a cotton wick and were checked at 24 hours and 48 hours after removal to assess mortality. In addition, a subsample of Petri dishes had been kept at constant $12^{\circ} \mathrm{C}$ over the entire experiment as a control to assess parasitoid quality.

Parasitoids allocated to the $-4^{\circ} \mathrm{C}$ treatment were chilled to the target temperature in a similar fashion; they were held at $6^{\circ} \mathrm{C}$ for 1 hour, $0^{\circ} \mathrm{C}$ for 1 hour and then cooled to $4^{\circ} \mathrm{C}(8 \mathrm{~L}: 16 \mathrm{D})$. Since a preliminary observation had shown survival to be substantially longer at $-4^{\circ} \mathrm{C}$ than at $-12^{\circ} \mathrm{C}$, samples of dishes were removed weekly from the cold treatment. Parasitoids removed from the freeze treatment were warmed and given sucrose solution as described for the $-12^{\circ} \mathrm{C}$ treatment.

As in previous experiments, a sub-sample of surviving females was set up in Petri dishes with sucrose solution and provided with hosts on a daily basis to evaluate the reproductive capacity of the parasitoids. These parasitoids were set up in pairs and given four fresh leek moth hosts per day for three days (=12 hosts). The hosts were subsequently monitored to record emergence of parasitoid offspring.

\subsection{Results}

\subsubsection{Developmental Thresholds and Thermal Budget}

Rearing temperature had a strong effect on the survival and development time of $D$. pulchellus $(F=59.85 ; P<0.001)$. Figure $3-2$ illustrates the relationship between temperature and the proportion of host pupae yielding an adult parasitoid. Clearly, the temperature range tested here pushed the upper and lower developmental limits of $D$. 
pulchellus. The optimal temperature range for offspring survival was $17.5^{\circ} \mathrm{C}$ to $22.5^{\circ} \mathrm{C}$. Survival was significantly reduced above and below this range. At the extremes, no parasitoids emerged from $32.5^{\circ} \mathrm{C}$ and $35^{\circ} \mathrm{C}$, while only 3 parasitoids emerged from a total of approximately 1750 potentially parasitised pupae $(=0.2 \%)$ stored at $7.5^{\circ} \mathrm{C}$.

Parasitoid development rate was strongly temperature-dependent, and generally increased with increasing temperature (Figure 3-3). A linear model effectively explained nearly all of the variation in development rate within the mid-range of temperatures (15$\left.27.5^{\circ} \mathrm{C}\right)\left(y=0.0039 x-0.0284 ; r^{2}=0.997 ; F=9110.0 ; P<0.001\right)$ and extrapolation leads to an estimated lower developmental threshold of $7.3^{\circ} \mathrm{C}$. This calculation fits very well with the observation that parasitoids emerged from only $0.2 \%$ of potentially parasitised pupae in the $7.5^{\circ} \mathrm{C}$ treatment. The thermal budget of $D$. pulchellus was estimated at 256.4 day-degrees.

When emerging females were set up in Petri dishes with males and subsequently given fresh hosts for oviposition, they all showed a strong response to the leek moth pupae. However, reproductive success was substantially reduced among females reared at the temperature extremes $\left(7.5,10\right.$ and $30^{\circ} \mathrm{C}$ ). In a haplo-diploid species like $D$. pulchellus, the production of female progeny demonstrates that males were capable of fertilising parasitoid eggs. These post-emergence oviposition trials demonstrated that females reared at all temperature treatments, except 7.5 and $30^{\circ} \mathrm{C}$, were able to produce both male and female offspring. Mating trials could not be conducted for the $7.5^{\circ} \mathrm{C}$ treatment since no males emerged at this temperature. Males and females reared at $30^{\circ} \mathrm{C}$ may have mated, but only a single progeny (male) was produced from 60 hosts provided for these females; hence, it was not possible to detect successful egg fertilisation.

\subsubsection{Effect of Variable Durations at Cool Temperatures on Pre-Imaginal Survival}

Under the test conditions, 3 and $8^{\circ} \mathrm{C}$ were lethal to developing $D$. pulchellus when the insects were subjected to the cold for extended periods of time (Figure $3-4$ ). At $3^{\circ} \mathrm{C}$, parasitoid emergence declined continuously with increasing cold exposure and stopped completely in the 7 and 10 -week treatments $(H=23.26 ; P<0.001)$. Longer durations at $8^{\circ} \mathrm{C}$ similarly meant reduced survival of parasitoids and none survived the 10 -week exposure $(H=22.58 ; P<0.001)$. In contrast, there were no significant differences in 
survival rates among the different treatments at $12^{\circ} \mathrm{C}(H=8.657 ; P=0.194)$. In fact, parasitoids in the 7 and 10-week exposures at $12^{\circ} \mathrm{C}$ were able to complete their development at that cool temperature and emerge well before the seventh week.

\subsubsection{Pre-Imaginal Development under Autumn Conditions}

Exposing parasitised pupae to natural autumn conditions apparently did not induce a successful pre-imaginal overwintering state in $D$. pulchellus. Parasitoid adults were observed emerging from their hosts at various points throughout the winter between the first week of December and the second week of February. Most emergence was recorded during mild periods in early December and then again from early to mid-January. As would be expected, the development of parasitoids was markedly retarded in the outdoor treatment compared to the $20^{\circ} \mathrm{C}$ control. With respect to the rate of parasitoid emergence among the cohorts in the outdoor treatment, there was a very distinct division based on date of parasitism (Figure 3-5). Those dishes containing pupae that were parasitised between 24 September and 3 October all had some degree of successful parasitoid emergence throughout the winter. In contrast, those dishes with pupae parasitised after 3 October had little or no emergence and there appears to be a steady decline in parasitoid emergence rates from pupae parasitised over the first three days of October. While parasitoid emergence rates in the $20^{\circ} \mathrm{C}$ treatment were visibly lower in dishes with pupae parasitised in early October, this change was not nearly as extreme as that observed in the outdoor treatment, suggesting a strong effect of the outdoor exposure.

Although less obvious, there was a significant treatment effect even among the cohorts at the start of the experiment. Using data from only the first cohorts (24-30 September) before the decline in survival in the outdoor treatment, the emergence rate at $20^{\circ} \mathrm{C}$ was $36.5 \pm 1.8 \%$ but only $27.0 \pm 1.7 \%$ in the outdoor treatment $(t=3.773 ; P<$ 0.001 ). As further evidence that this difference is not simply due to variable parasitism rates, the proportion of pupae yielding healthy leek moth adults was virtually identical between treatments $(t=-0.091 ; P=0.928)$. This means that parasitoid-induced mortality, which accounts for nearly all mortality under laboratory conditions, was consistent (68.3 $\pm 2.8 \%$ at $20^{\circ} \mathrm{C}$ and $68.6 \pm 2.7$ outside). Therefore, it appears that equal numbers of 
pupae were parasitised in the paired cohorts, resulting in similar rates of leek moth mortality, but fewer parasitoid offspring survived development under winter conditions.

As seen in a previous experiment, parasitoids that had been reared at $20^{\circ} \mathrm{C}$ produced both male and female offspring. In contrast, the parasitoids that had emerged in the outdoor treatment showed very low oviposition success. Only 2 of 10 groups of females produced any offspring and, of these, only 1 produced females. While this does confirm that winter-emerging females were mated and capable of mating and reproducing, they appear to be in a less fit state for reproduction than their siblings reared under summer conditions. The poor physical condition of parasitoids reared outdoors was often plainly visible in that they were lethargic even several days after being brought into the laboratory and fed a $20 \%$ sucrose solution.

\subsubsection{Adult Overwintering Success}

Overall, females survived the 3-month cold treatment better than males (Figure 36). Males only survived in treatments where the temperature never dropped below freezing. In contrast, there was some female survival in all four treatments, albeit very low in the $-1{ }^{\circ} \mathrm{C}$ and outdoor treatments. Overwintering survival was highest among females in the $1^{\circ} \mathrm{C}$ treatment, followed by females in the treatment with temperatures shifting between 1,3 and $6^{\circ} \mathrm{C}(F=21.2 ; P<0.001)$. Surviving females from all overwintering regimes were capable of producing both male and female offspring, verifying that mating did occur either immediately after emergence or during cold conditioning at $12^{\circ} \mathrm{C}$ before the sexes were separated.

\subsubsection{Adult Freeze Tolerance (LTime 50 )}

Diadromus pulchellus males and females showed varying degrees of cold hardiness. As observed in the previous overwintering experiment, females tended to have higher survival rates than males. Although mortality was assessed 24 and 48 hours after parasitoids were removed from the cold treatment, mortality was apparent in all but a few cases after the first 24 hours. For males and females reared at $22^{\circ} \mathrm{C}$, the LTime 50 is $4-5$ days and 6-7 days, respectively (Figure 3-7a). Parasitoids reared under autumn conditions 
(Figure 3-7b) seem to be slightly more cold tolerant, however, this difference cannot be tested statistically as there was only a single replicate for cold-reared parasitoids.

Parasitoid survival was substantially longer in the $-4^{\circ} \mathrm{C}$ freeze treatment. The LTime $_{50}$ for males reared under summer conditions was between 7 and 14 days (Figure 3-8). Only a single male was still alive after 4 weeks and this was the maximum survival time recorded. The mortality of summer-reared females approached $50 \%$ the second and third weeks at $-4^{\circ} \mathrm{C}$ (Figure 3-8). The longest surviving of these female was removed from $-4^{\circ} \mathrm{C}$ after 8 weeks while no females survived the 9- and 10-week exposures. As in the $-12^{\circ} \mathrm{C}$ treatment, parasitoids reared under autumn conditions appeared to be slightly less vulnerable to freeze mortality (Figure 3-9). Cold-reared males showed higher survival after 2 weeks than summer-reared males. Similarly, the LTime 50 of females reared under autumn conditions (21-28 days) was one to two weeks later than that of females reared at $22^{\circ} \mathrm{C}$. A few of the cold-reared females managed to survive 9 weeks of freezing, but none was alive after 10 weeks.

Female parasitoids that had been reared under summer conditions were capable of attacking leek moth hosts and producing both male and female offspring when provided with hosts after the freeze treatment. Those parasitoids reared outdoors prior to the freeze tests had much lower reproductive success, but were still able to produce some offspring. This lower success in the latter group is not surprising since offspring production was already observed to be lower among females reared outdoors during late autumn.

\subsection{Discussion}

There has been a general sense among classical biological control practitioners that the effect of environmental factors, such as climate and the availability of overwintering sites, on the establishment success of agents cannot easily and accurately be predicted in advance of actual field releases (Van Driesche and Hoddle, 2000). This is due to a lack of knowledge of the relationships between results from laboratory cold hardiness tests and field overwintering survival. As a result, classical biological control has generally involved releasing large numbers of agents and conducting post-release surveys to verify that establishment is possible. More recently however, research has focused on 
identifying laboratory indices of cold hardiness that correlate with the duration of winter survival under field conditions. After comparing results from several laboratory cold response tests with results from outdoor overwintering trials, Hatherly et al. (2005) found a strong correlation between the laboratory LTime $5_{00}$ at $5^{\circ} \mathrm{C}$ and the maximum field survival time of exotic predatory mites in the UK. While this survival model is defined by only a few related case studies and is specific to mites in a region with winter temperatures around $5^{\circ} \mathrm{C}$, it demonstrates that simple laboratory tests may yet emerge as effective surrogates of actual field overwintering trials for candidate biological control agents.

In the current study, a number of experiments were run to try to identify the overwintering stage and measure the cold hardiness of Diadromus pulchellus, a candidate classical biological control agent for North America. The first experiment determined the lower and upper developmental thresholds as well as the thermal budget of the agent. An introduced species will only survive if it has a sufficient thermal budget above its lower developmental threshold to complete its life cycle, reproduce and survive the winter. In eastern Ontario and western Quebec there are enough day-degree units between March and September to easily allow three generations of parasitoids. Based on heat accumulation alone, $D$. pulchellus could probably undergo as many as four or five generations; however, the parasitoids would likely experience only three due to synchrony with leek moth phenology and the periodic availability of hosts. Leek moth phenology in Ontario (Mason et al., 2006) closely matches that observed in Switzerland, with the appearance of leek moth pupae in mid- to late-May, mid-July and mid- to lateAugust. Thus, there should be no problems with synchronisation between $D$. pulchellus populations from central Europe and the invasive leek moth populations in Canada. The first experiment also showed that the lower developmental threshold for D. pulchellus is $7.3^{\circ} \mathrm{C}$, while Mason et al. (2006) calculated a threshold of approximately $7.0^{\circ} \mathrm{C}$ for leek moth. This indicates that the parasitoid is capable of developing under virtually any cool conditions where leek moth can develop. Moreover, the optimal rearing temperature for D. pulchellus survival was between 17.5 and $22.5^{\circ} \mathrm{C}$ and the mean daily temperatures for June, July and August in the proposed release region fall within this range (Environment Canada, 2008). 
The second and third experiments examined how parasitoids developing in their hosts responded to autumnal conditions. These tests demonstrated that immature parasitoids died when exposed to sustained cool temperatures $\left(3\right.$ and $\left.8^{\circ} \mathrm{C}\right)$ or fluctuatng temperatures that repeatedly fell below freezing. In the outdoor overwintering trials, the sudden decline in parasitoid emergence from pupae parasitised in October (Figure 3-5) suggests that the last cohorts of pupae placed outside were subjected to more lethal conditions. A phase of freezing temperatures occurred in mid-November before the first parasitoid emergence in early December so this demonstrates that it is possible for immature parasitoids to survive relatively short periods of sub-zero temperatures. It also means that identifying a specific cold event as the cause of mortality is not feasible. Even the longest and coldest period (late December) did not kill all parasitoids as there was subsequent emergence once temperatures rose in mid-January. A likely explanation for the lack of emergence from the last cohorts is that the colder ambient temperatures in mid-October and mid-November were harder on those immature parasitoids that were in earlier stages of development (i.e., the last cohorts produced in October). By the time the temperatures began to fall to around $0^{\circ} \mathrm{C}$, parasitoids in the older cohorts may have had time to reach a critical stage to withstand such harsh conditions. This study did not attempt to identify which pre-imaginal stages were most cold hardy, however, it is likely that most of the winter mortality occurred during the larval, and possibly pupal, stages. Kalmes (1984) noted that all D. pulchellus eggs hatch within 72 hours under a $25^{\circ} \mathrm{C} / 15^{\circ} \mathrm{C}$ thermoperiod and within 48 hours under a $30^{\circ} \mathrm{C} / 20^{\circ} \mathrm{C}$ thermoperiod. Since parasitised pupae in the second experiment (sustained cool temperatures) were stored at $22^{\circ} \mathrm{C}$ for 72 hours before being placed into constant cool temperatures, it is certain that all eggs would have hatched and the mortality observed at 3 and $8^{\circ} \mathrm{C}$ would have therefore occurred in the larval or pupal stages.

Based on these results, it is quite unlikely that $D$. pulchellus truly overwinters in an immature stage, even though these pre-imaginal stages appear to tolerate freezing temperatures. Nonetheless, an immature overwintering stage cannot be completely ruled out. One important factor not tested in these trials was a possible maternal effect on diapause induction. While most insect species are induced to diapause by subjecting the overwintering individual to the appropriate cues, there are some species in which factors 
affecting the maternal generation induce diapause in the progeny (Saunders, 1965). In the experiments described here, all ovipositing females had been reared under standardised summer conditions and therefore would not have induced their offspring to diapause. Hence, this is one aspect that could be given further attention.

Despite the uncertainty around the ability of $D$. pulchellus to overwinter in a preimaginal stage, this agent is suspected to overwinter primarily, if not exclusively, as an adult. This concept is based on a number of lines of evidence. First, five congeneric species ( $D$. arrisor, $D$. candidatus, $D$. collaris, $D$. intermedius and $D$. troglodytes) have all been recorded to overwinter as adults (Rasnitsyn, 1964; Valemberg and Vago, 1974; Sebald et al., 2000; Bauer, 2001), whereas there are no reports of Diadromus spp. overwintering inside their hosts. According to Danks (1978), the overwintering stage of a species is usually consistent within a genus and sometimes within a higher taxon. Indeed, adult overwintering is also known from other species in the tribe Alomyini, of which Diadromus is a member.

Second, there is no evidence from the field to suggest that D. pulchellus remains inside its host at the end of the final leek moth generation. When third generation leek moth pupae are collected from the field in late August and early September, both leek moth and $D$. pulchellus adults emerge from the cocoons no differently than in the previous two generations. Furthermore, female parasitoids emerging at the end of the season do not appear to be in a diapausing state, as they mate readily and will oviposit if presented with fresh hosts. In a natural setting, however, there do not seem to be any available hosts at this point in the season. Unlike D. pulchellus, third generation leek moths emerge in a state of reproductive diapause, induced by cues detected during their development (Thibout, 1981; Abo-Ghalia and Thibout, 1982). Hence, there should normally be no option for oviposition after the third leek moth generation. One exception that was observed in the field in Switzerland was the appearance of leek moth pupae in late September and early October. This was almost certainly due to a severe delay in the completion of the third generation due to unusually cold weather in August and September. Thus, it was possible for oviposition to occur after the emergence of third generation parasitoids. However, no parasitism was detected in the few pupae collected from the field in late September. Even if there had been parasitised pupae, the parasitoids 
would likely have emerged at some point in the early winter, as demonstrated in the third experiment described above, and overwintered as adults.

Finally, $D$. pulchellus adults are relatively long-lived and show resilience to cold temperatures. Preliminary tests showed that, when provided with a sucrose solution, these parasitoids can survive for up to 9 or 10 weeks at $20^{\circ} \mathrm{C}$ and for over 12 weeks at temperatures around $10^{\circ} \mathrm{C}$. Both male and female parasitoids survived an overwintering phase with temperatures above freezing and a few females were still alive following the same trial period under $-1{ }^{\circ} \mathrm{C}$ and natural outdoor conditions. As the specific behavioural response of $D$. pulchellus to decreasing temperatures is not known, it is not certain whether the small experimental cages accurately simulated a typical overwintering site for this species. For instance, there was evidence of burrowing into the soil at the bottom of the cages, however this soil may have been far too shallow to offer sufficient buffering against harsh temperatures. If the parasitoids had been allowed to select their own overwintering sites within a larger patch of more variable habitat, it is quite likely that survival would have been enhanced, particularly if the parasitoids had had the freedom to move freely between sites during warmer periods.

In addition to the degree of overwintering success, $D$. pulchellus demonstrated the capability to tolerate extended periods of sub-zero temperatures. Females were able to survive up to nine consecutive weeks at a constant $-4^{\circ} \mathrm{C}$, which is considered to be an approximation of winter temperatures at ground level beneath a layer of snow. In instances where parasitoids might find themselves without shelter during a cold phase with temperatures around $-10^{\circ} \mathrm{C}$, they would be able to survive for up to one week. It is important to note that the parasitoids in the freeze trials were stored in empty Petri dishes during the cold treatment. The addition of shelters in such trials may greatly improve survival by protecting the parasitoids against relatively rapid temperature changes.

Although there are insufficient data to use the LTimes 50 as a predictive index the way Hatherly et al. (2005) demonstrated, it is still useful particularly when it is possible to predict natural winter temperatures. Fortunately, the ground temperatures beneath a solid layer of snow tend to be quite constant and predictable to within a couple of degrees. Thus, the LTime $_{50}$ tests at $-4^{\circ} \mathrm{C}$ were likely fairly accurate simulations of temperatures to be encountered by parasitoids overwintering in leaf litter. Furthermore, 
empirical tests such as these will contribute to expanding upon predictive models like the one developed by Hatherly et al. (2005).

In conclusion, the results of this study suggest that $D$. pulchellus parasitoids likely overwinter in the adult stage as has been described for some congeneric species. This is not surprising since there do not tend to be overwintering host pupae available at the end of the season due to the leek moth's adult-overwintering strategy. This is also an important observation with respect to host specificity of the agent since it would rule out the need for an alternative overwintering host. The immature stages are also capable of withstanding at least short periods of temperatures as low as -5 or $-10^{\circ} \mathrm{C}$, therefore early frosts may not pose a great risk to the survival of the final generation in late August and September. Cold hardiness was more apparent in adult females and this should result in female-biased populations in the spring. However, because these parasitoids are reproductively active upon emergence in the fall, it is likely that a large proportion of the population would be mated prior to the onset of winter. Overwintered females readily oviposited into fresh hosts within three days of being warmed to $20^{\circ} \mathrm{C}$. In light of the apparent cold hardiness of $D$. pulchellus adults and immature stages, it is likely that this species could endure winters in Ontario and Quebec, provided that their requirements for overwintering sites would be met (Danks, 1978). 


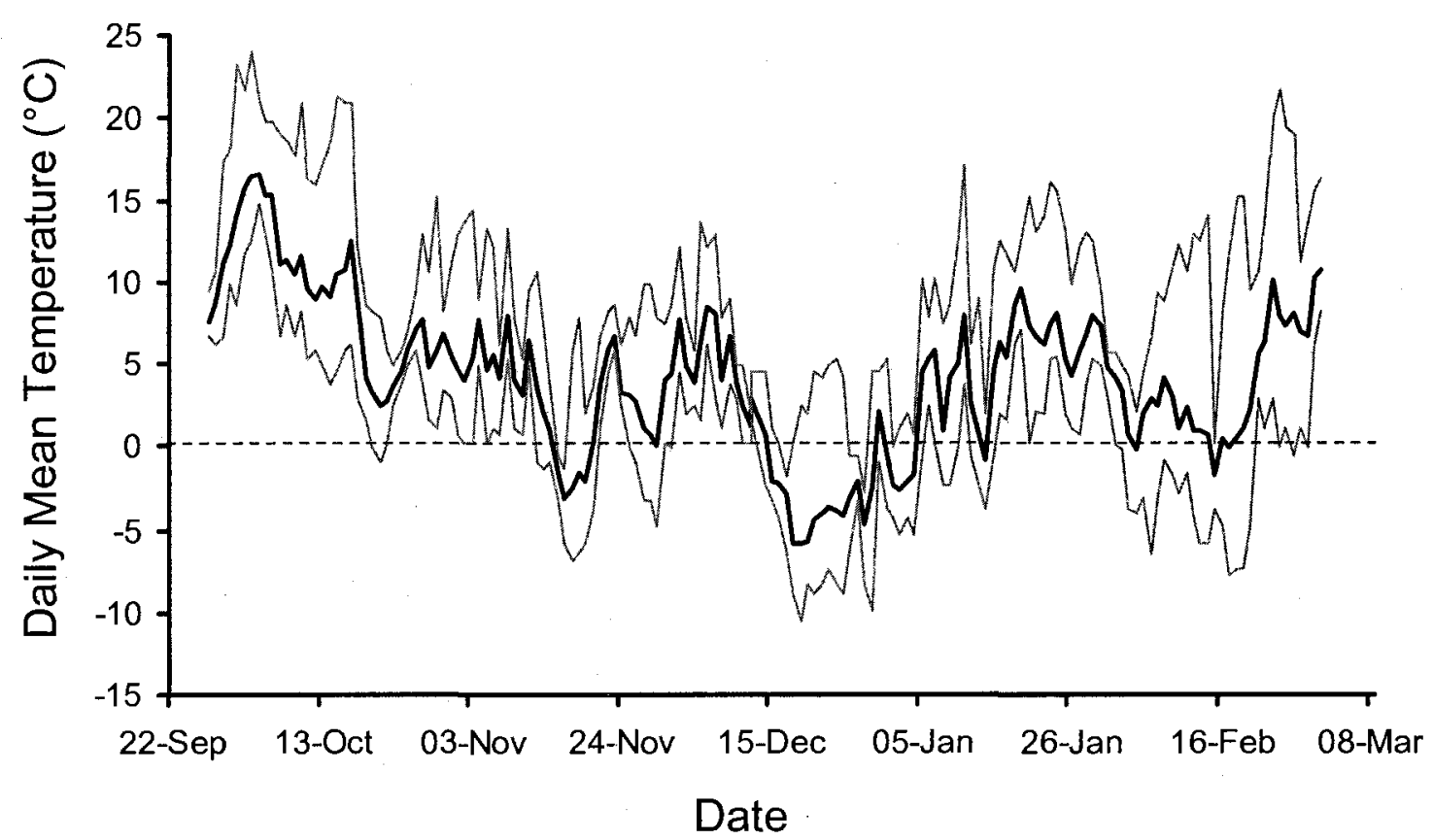

Figure 3-1. Recorded temperatures from late September to early March inside a shaded and open-ended poly-ethylene tunnel used for overwintering trials with both immature and adult Diadromus pulchellus Wesmael. This tunnel was located in the experimental garden of CABI Europe-Switzerland. Bold line represents daily means while grey lines show daily minimum and maximum temperatures. Horizontal dashed line shows the freezing point. 


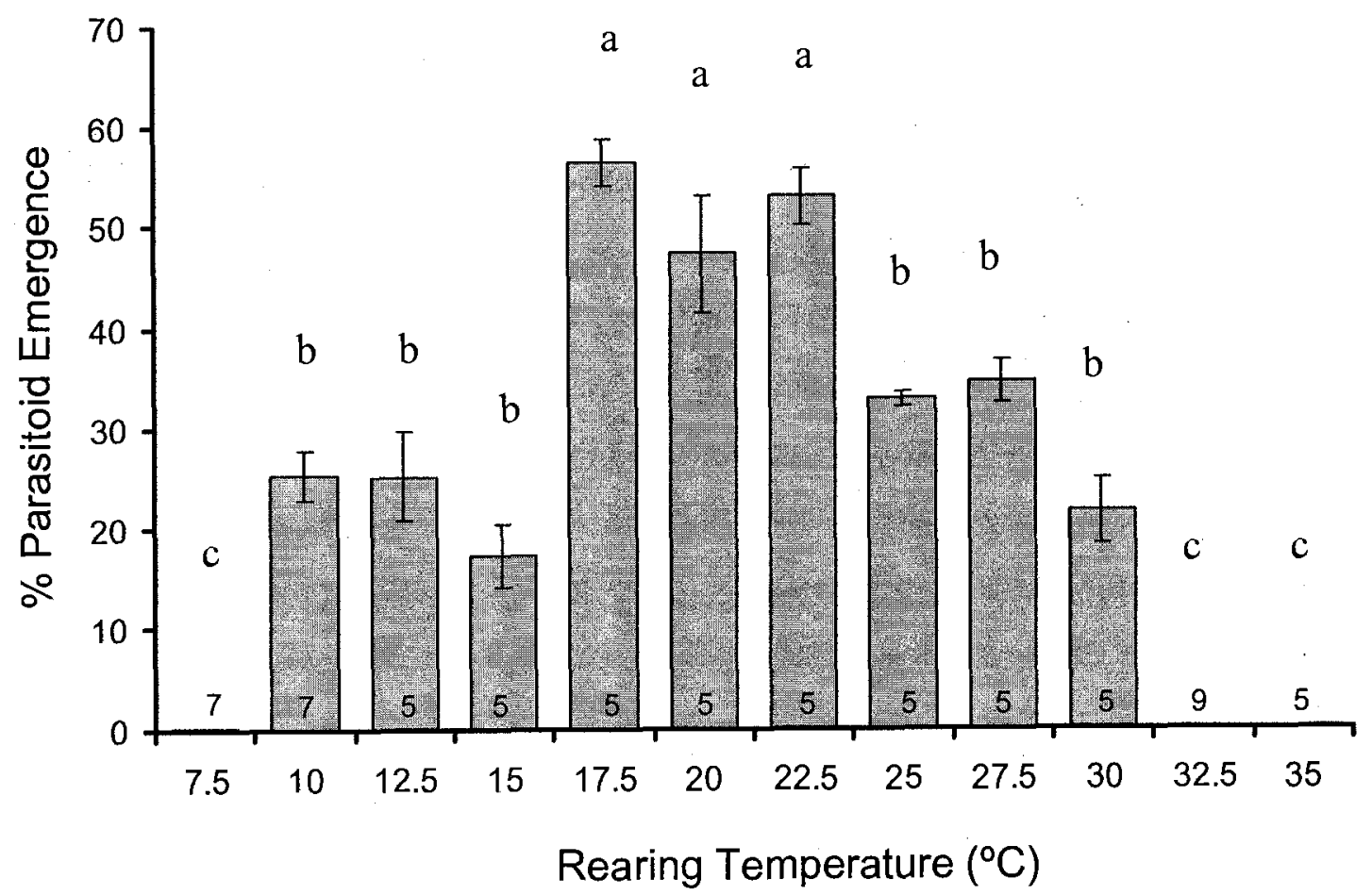

Figure 3-2. Emergence rates ( $\% \pm$ S.E.) of Diadromus pulchellus Wesmael reared from egg to adult at different constant temperatures. The number of replicates per temperature treatment is indicated at the base of each column. Columns with different letters have significantly different means. 


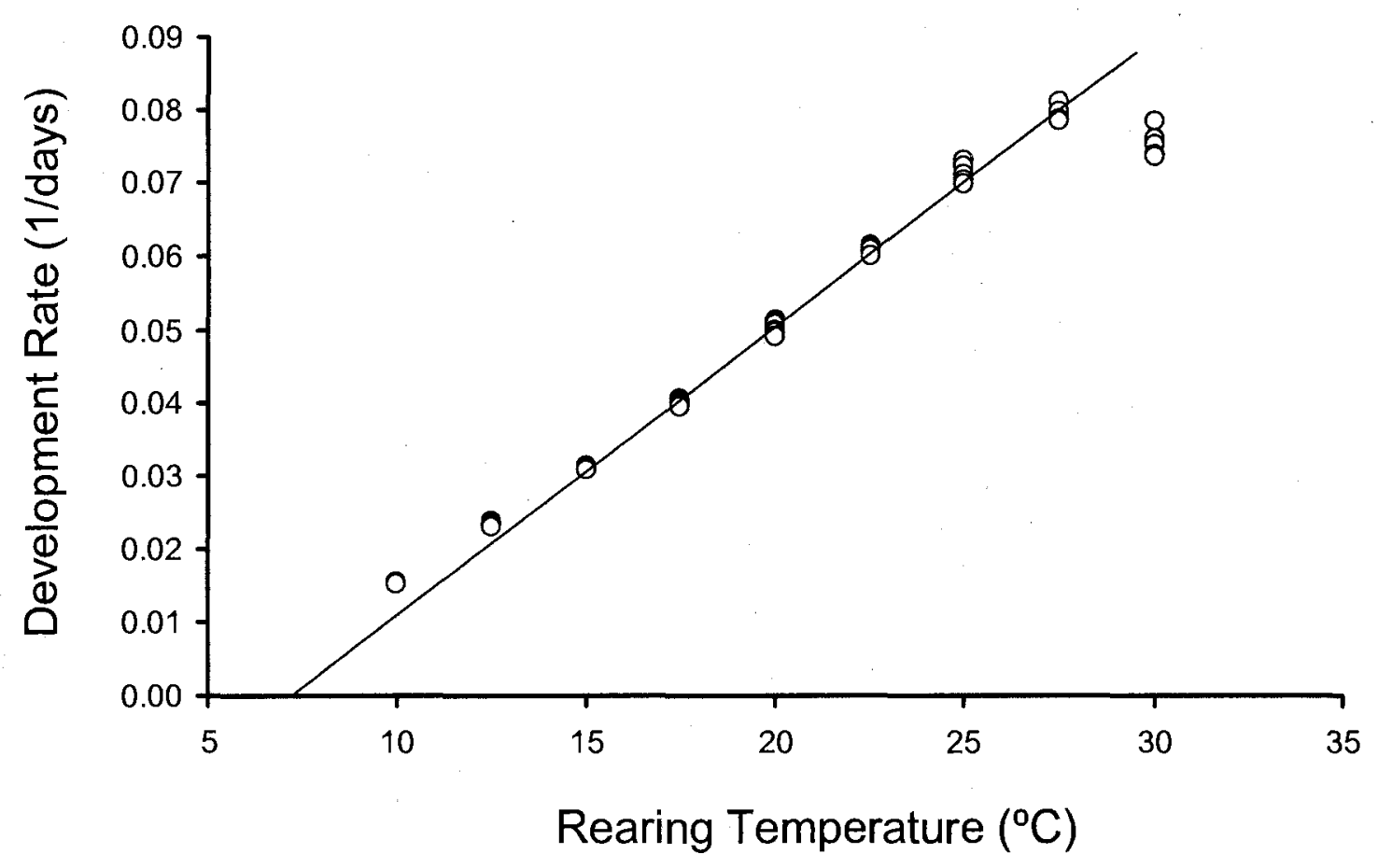

Figure 3-3. Development rate of Diadromus pulchellus Wesmael reared from egg to adult at different constant temperatures. Line is fitted with simple linear regression (including only mid-range data points: 15 to $27.5^{\circ} \mathrm{C}$ ). Through extrapolation, the lower temperature threshold is calculated as $7.3^{\circ} \mathrm{C}$. 

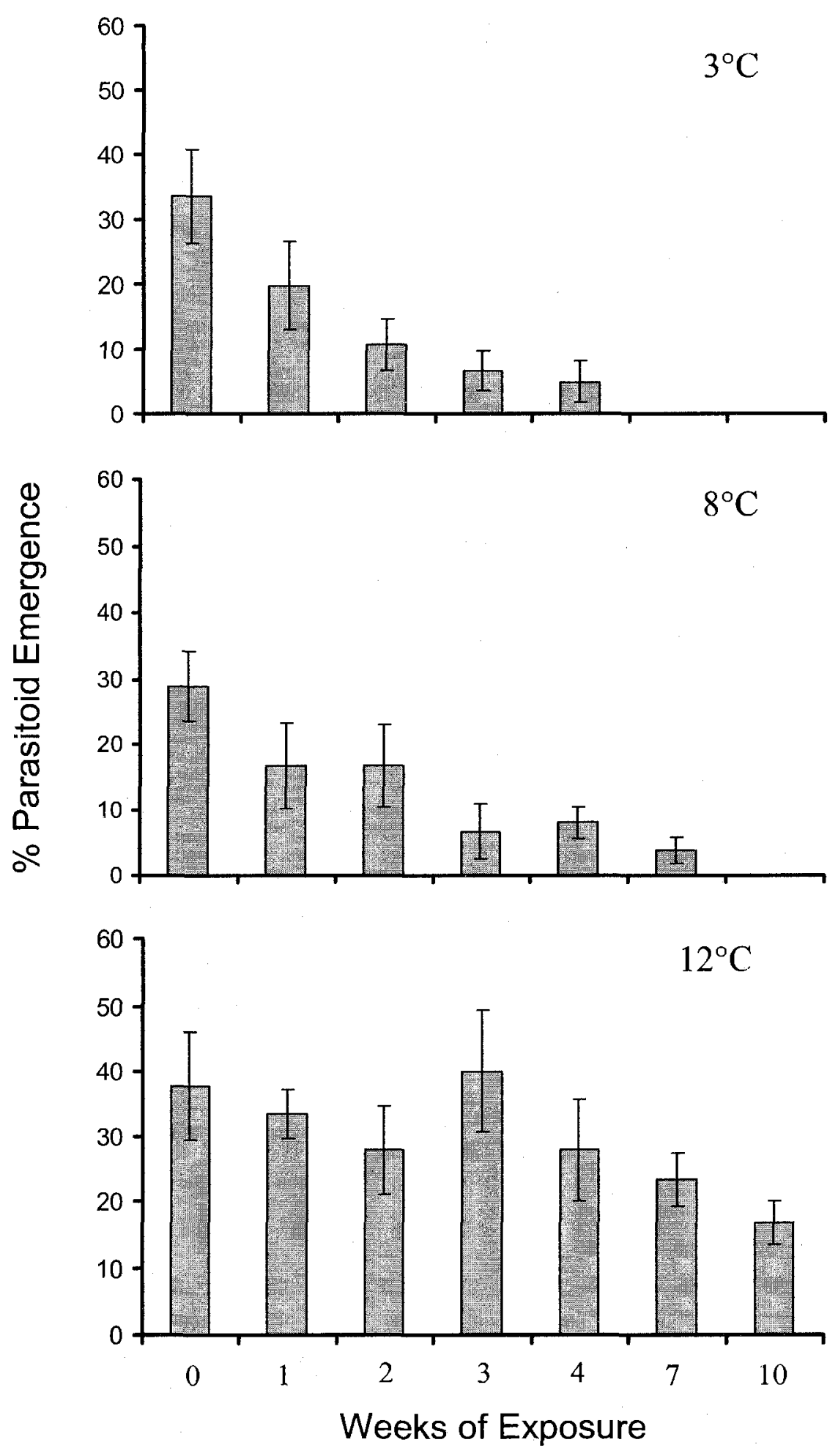

Figure 3-4. Percentage of parasitised pupae ( $\% \pm$ S.E.) yielding healthy Diadromus pulchellus Wesmael offspring following varying durations ( 0 to 10 weeks) at $3^{\circ} \mathrm{C}, 8^{\circ} \mathrm{C}$ and $12^{\circ} \mathrm{C}$. All exposure durations for each temperature treatment were replicated 6 times. 


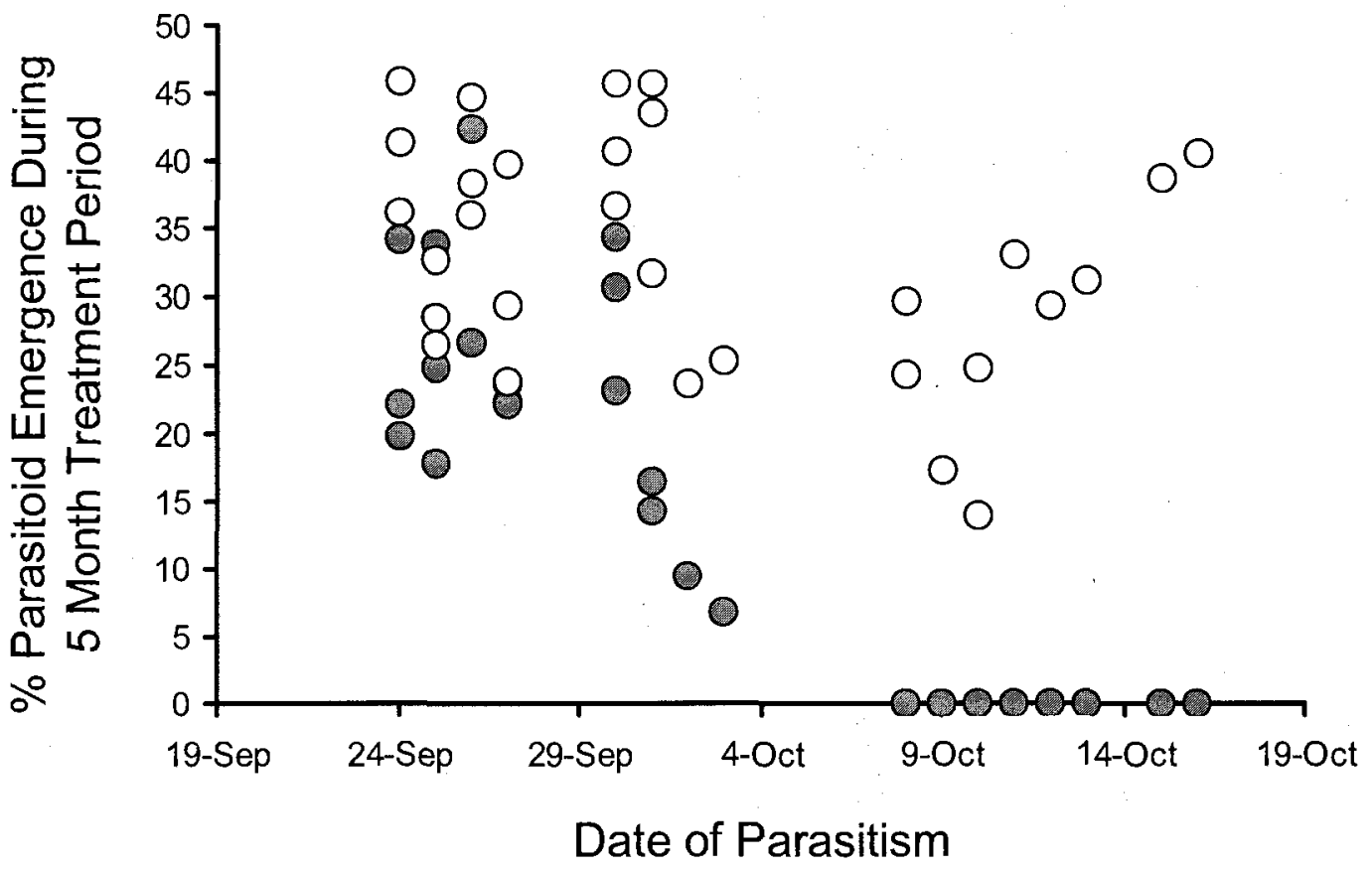

Outdoor treatment

Control

Figure 3-5. Emergence rate of Diadromus pulchellus Wesmael adults during a five-month treatment period as a function of the date of parasitism. Each pupal cohort was placed in either the outdoor treatment or the $20^{\circ} \mathrm{C}$ control immediately after exposure to parasitoids at room temperature. The percent emergence includes all parasitoids that emerged from a particular host cohort over the course of the entire winter treatment. 


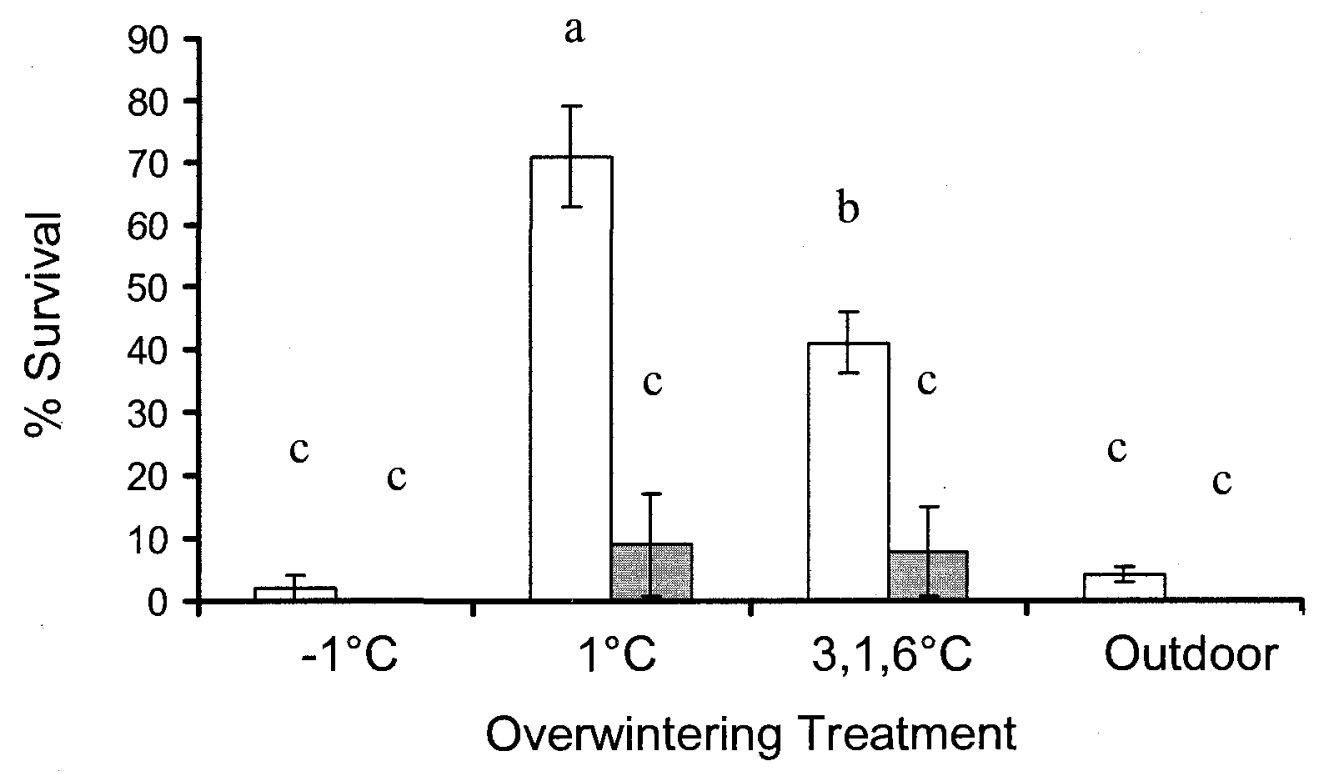

Female D. pulchellus

Male D. pulchellus

Figure 3-6. Survival (\% \pm S.E.) of female and male Diadromus pulchellus Wesmael held for 3 months under different overwintering treatments. The " $3,1,6^{\circ} \mathrm{C}$ " treatment consisted of constant $3^{\circ} \mathrm{C}$ for four weeks, then constant $1{ }^{\circ} \mathrm{C}$ for two weeks and then constant $6^{\circ} \mathrm{C}$ for the final six weeks. Each treatment was replicated 5 times for both males and females. Columns with different letters are significantly different from one another. 

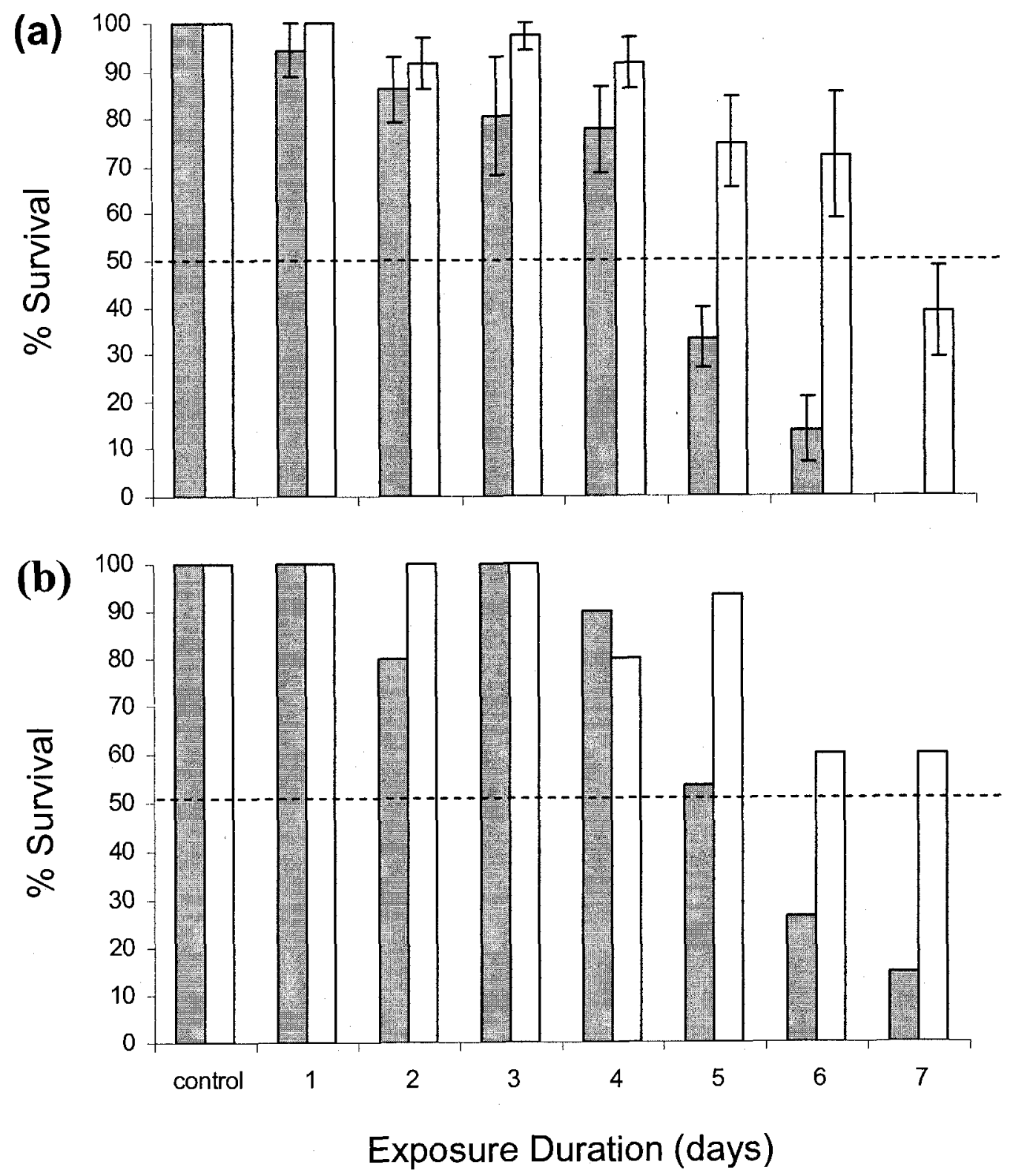

Male D. pulchellus

Female D. pulchellus

Figure 3-7. Survival ( $\% \pm$ S.E.) of male and female Diadromus pulchellus Wesmael adults subjected to varying durations of exposure to $-12^{\circ} \mathrm{C}$ to determine the time required to kill $50 \%$ of a cohort. Freeze trials were run with parasitoids (a) reared from egg to adult under summer conditions $\left(22 \pm 2^{\circ} \mathrm{C}\right)$ (replicated 4 times) as well as (b) those emerging from pupae that had been placed outside for the overwintering trial (single replicate only). Parasitoids in the "control" treatment were kept at constant $12 \pm 2^{\circ} \mathrm{C}$. 


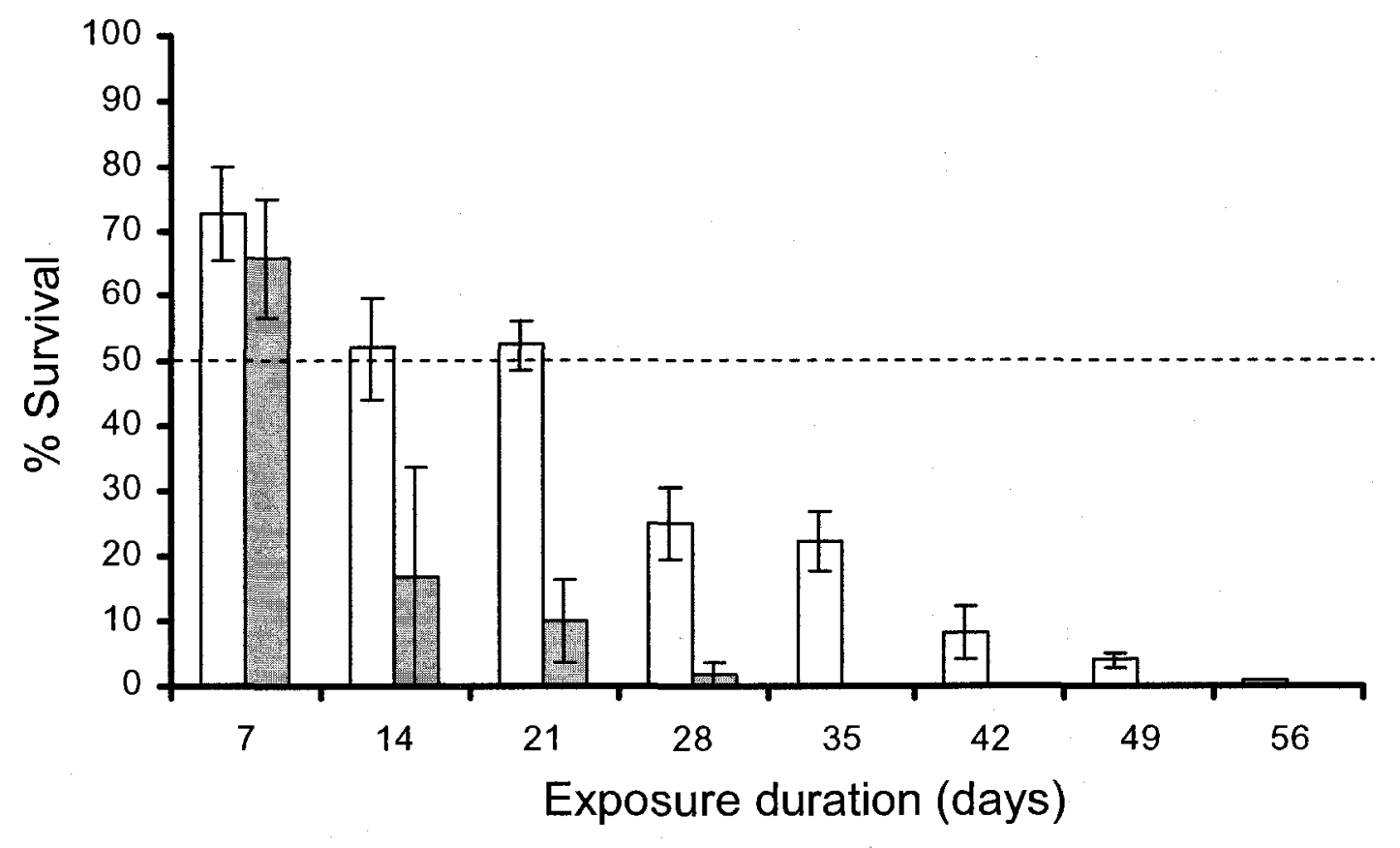

\section{Male D. pulchellus $\quad \square$ Female $D$. pulchellus}

Figure 3-8. Survival $(\% \pm$ S.E.) of male and female Diadromus pulchellus Wesmael subjected to varying durations of exposure to $-4^{\circ} \mathrm{C}$ to determine the time required to kill $50 \%$ of a cohort. All trials represented here were run with parasitoids reared from egg to adult under summer conditions $\left(22 \pm 2^{\circ} \mathrm{C}\right)$. Each exposure duration was replicated 7 times. 


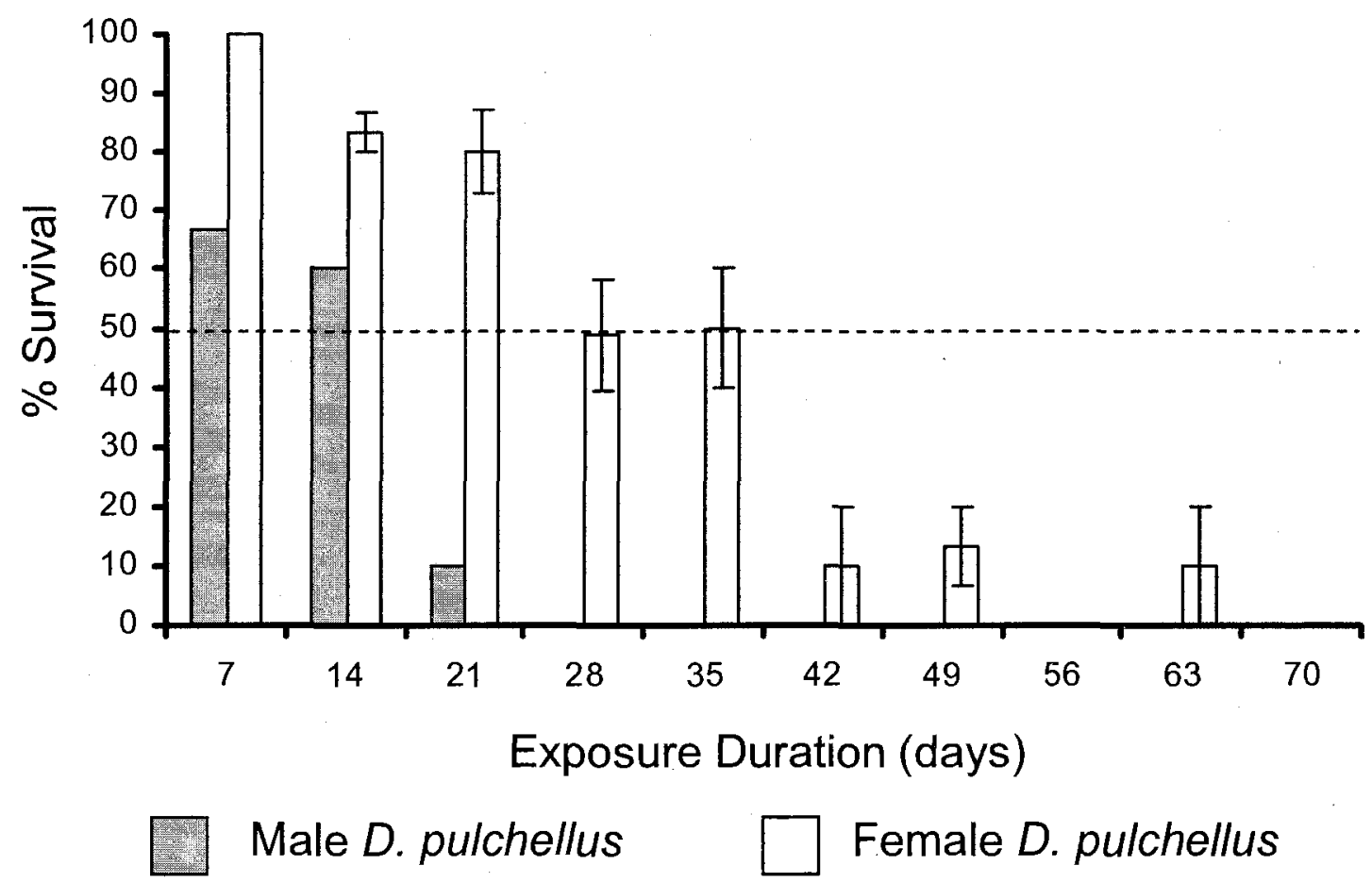

Figure 3-9. Survival (\% $\%$ S.E.) of male (single replicate only) and female (replicated 3 times) Diadromus pulchellus Wesmael subjected to varying durations of exposure to $4^{\circ} \mathrm{C}$ to determine the time required to kill $50 \%$ of a cohort. All trials represented here were run with parasitoids reared from egg to adult under natural autumn and winter conditions. 


\section{Effect of Physiological State and Environment on a Parasitoid's Motivation to Oviposit}

\subsection{Introduction}

There are two fundamental questions concerning a candidate agent for classical biological control: (1) Will the agent be effective in reducing pest numbers? (2) Will the agent pose any risk to non-target species if it is introduced into a new territory? In most modern biological control programmes, it is the latter question that receives the greatest attention and resources due to the challenges associated with confidently clarifying an agent's host range (Babendreier et al., 2006). While the indirect and long-term ecological effects of biological control can be especially difficult to identify and quantify (Hopper $e t$ al., 1993; Roitberg, 2000; Messing et al., 2006), direct non-target effects can be estimated using trials in which the agent is offered any number and combination of a variety of hosts or prey belonging to species other than the target (Van Driesche and Murray, 2004). Variants of this method of non-target testing have been applied to weed biological control agents for several decades and, to a growing extent, to entomophagous agents in the last decade.

Pre-release host range tests can, at best, give only an approximation of an agent's ecological host range (= the set of species used for reproduction in the field (Nechols et al, 1992; Onstad and McManus, 1996)). This is due to a number of limitations of host range testing, including restricted sample sizes, the limited number of species that can feasibly be tested due to collection and rearing challenges, a lack of knowledge regarding the distributions of and phylogenetic relationships between non-target species, the lack of realism in many test designs and the difficulty of forecasting longer-term shifts or expansions of host range (Nechols et al, 1992; Secord and Kareiva, 1996; Hopper, 2001; Van Driesche, 2004). Relatively recent studies have evaluated the accuracy of laboratorybased host specificity tests by comparing results from such studies with actual patterns of non-target attack in the field (Barratt et al., 1997; Haye et al., 2005). These studies demonstrated that laboratory trials were indicative of non-target parasitism in the field, but likely overestimate the ecological host range and provide little or no reliable 
information on quantitative effects in the field. Nonetheless, given the typically enormous task of determining ecological host range directly from the field, van Lenteren et al., (2006) advised that an appropriate first step to non-target risk assessment is to measure the fundamental host range ( $=$ the set of all species that can support development of the agent (Nechols et al, 1992; Onstad and McManus, 1996)).

Given the discrepancy between fundamental and ecological host ranges, there is building pressure to design and implement testing protocols that minimise the probability of false-negative results (i.e., results that incorrectly identify a non-target as unsuitable (sensu Marohasy, 1998)). It is generally felt that during host specificity testing it is better to err on the side of safety and risk obtaining false-positive results (i.e., results that incorrectly mark a non-target as a likely host). Then, if there is suspicion that a testing scheme exaggerates the ecological host range of an agent, additional tests can be conducted under more natural conditions to verify whether the non-target is still attacked (van Lenteren et al., 2006). Withers and Browne (2004) argued that special consideration should be given to the agent's physiological and informational state, as well as to the test environment, in order to maximise the likelihood that a non-target will be accepted for oviposition.

Insect oviposition behaviour is regulated by endogenous factors that can cause variability in the behavioural responses of two or more individuals, or a single individual over time, despite subjecting them to identical environmental conditions (Papaj and Rausher, 1983; Browne, 1993; Briggs et al., 1999; Roitberg, 2000). Similarly, a shift in environmental cues can lead to significant changes in foraging behaviour (Roitberg et al., 1993). With respect to parasitoids, the forager's condition has repeatedly been shown to affect various aspects of search behaviour such as locomotor activity, responsiveness to odours, parasitism and superparasitism rates, host preferences and search efficiency (e.g., Lewis et al., 1990; Eller et al., 1992; Bjorksten and Hoffmann, 1995; Michaud and Mackauer, 1995; Pompanon et al., 1999; Daza-Bustamante et al., 2002). Nonetheless, despite over two decades of research into the effects of physiological and informational state on parasitoid performance from a theoretical perspective, careful consideration of endogenous factors during specificity tests of entomophagous agents is a recent phenomenon (Roitberg, 2000; Withers and Browne, 2004). 
Most importantly for the context of host range testing, an individual's "ovipositional drive" or "motivation to oviposit" can be enhanced under certain states (van Lenteren, 1976; Iwasa et al., 1984; Withers and Browne, 2004). Female parasitoids are generally expected to prefer ovipositing in high-quality hosts since the fitness of their offspring is strongly dependent on host quality. However, relatively low-quality hosts are also occasionally parasitised. Foraging theory predicts that this should occur when a parasitoid's acceptance threshold decreases sufficiently due to the perception of a low probability of successfully locating a more optimal host (Godfray, 1994). Since an insect's host acceptance threshold is not fixed (Browne and Withers, 2002), it should be possible to manipulate it.

An implicit assumption in host range testing is that agents used in laboratory trials will behave similarly to conspecific individuals in the field. Under field conditions, however, parasitoids would almost certainly be subjected to a wider range of experiences and stress factors and the result would be greater variance in the physiological and informational states among the individuals in a population. As it is impossible to replicate the full spectrum of all these states in the laboratory, Withers and Browne (2004) recommended manipulating the condition of agents to put them in a state where they are most likely to express the widest host range possible. Certain physiological and informational states (e.g., host deprivation, food deprivation and increased parasitoid density) appear to generally increase the ovipositional drive of parasitoids and are thus expected to increase the likelihood of attack on non-target hosts ( $=$ increased host range expression) (Withers and Browne, 2004). However, the effect of certain other variables, such as oviposition experience and the type of test, on a parasitoid's tendency to accept low-quality hosts is less clear (e.g., Bueno et al., 1993; Kitt and Keller, 1998).

Due to the relative novelty of this specific application of biological control theory, there is a need for empirical studies to explicitly test some of the associated assumptions. For example, Withers and Browne's (2004) suggestion that specific physiological factors could have predictable effects on a parasitoid's motivation to oviposit was based on studies that did not directly measure the effects of parasitoid state on oviposition activity. They assumed that a change in physiological state that modifies one behavioural parameter, such as responsiveness to odours or search efficiency, would also induce a 
change in the parasitoid's motivation to oviposit. Second, it is assumed that the direction and magnitude of change in one behavioural parameter will translate to a roughly equivalent transition in the probability of an oviposition event occurring. Thus, the prediction would be that if a parasitoid exhibits, for example, more direct flight toward an odour source, then it would also be more likely to oviposit into a host upon discovery. It is possible, however, that various behavioural parameters will not necessarily be so linearly correlated. In fact, it is conceivable that these relationships may sometimes be of an inverse nature. Therefore, while Withers and Browne (2004) make an important theoretical argument for host range test design, a generalised protocol may not accurately identify the "ideal state" of an agent for non-target testing.

A current classical biological control project for a vegetable pest was used as a model system to test whether the effects of physiological and informational state on motivation to oviposit are indeed predictable. The leek moth, Acrolepiopsis assectella (Zeller) (Lepidoptera: Acrolepiidae), is an exotic invasive species in North America. The larvae of this Palaearctic moth mine the leaves and stems of Allium spp., including cultivated species such as leeks, onions, garlic and chives. Since its discovery in Ottawa, Ontario in 1993 (Landry, 2007), leek moth has demonstrated continuous range expansion and is now considered to be a threat to certain elements of Allium production in Canada as well as native North American Allium species (Allison et al., 2007). The agent of interest is the pupal endoparasitoid, Diadromus pulchellus Wesmael (Hymenoptera: Ichneumonidae). Experiments were designed to explore whether variation in four physiological and informational states could significantly influence the motivation of $D$. pulchellus females to oviposit and whether the direction of the effects fit with Withers and Browne's (2004) predictions. This study specifically measured how the degree of host exploitation by parasitoids over a 24 -hour period was affected by female mating status, nutritional status, age and previous host experience. It also assessed the importance of the parasitoid's foraging environment by testing the effects of parasitoid density, arena size and the presence/absence of the host plant. 


\subsection{Materials and Methods}

\subsubsection{Insect Cultures}

A laboratory colony of $D$. pulchellus was established with parasitoids reared from leek moth pupae collected in organic and integrated production leek fields in west-central Switzerland in 2005. Prior to the experiments described below, multiple generations had been reared continuously in the laboratory and field-collected specimens were added to the culture each summer. Emerging parasitoids were stored in $30 \mathrm{~cm}^{3}$ plastic cages (BugDorm-1; BioQuip ${ }^{\circledR}$, Rancho Domingo, CA, USA) and provided with a $20 \%$ sucrose solution. Leek moth hosts were mass-produced on potted leeks in the laboratory. Each day, all newly formed cocoons containing pre-pupae were collected from the rearing cages and stored for one day to allow pupation to take place. This collection system ensured that the precise age of all hosts was known. The newly formed pupae not needed for oviposition experiments were offered en-masse to D. pulchellus in the BugDorms for oviposition to maintain the parasitoid culture. Following 24 hours of exposure, the parasitised pupae were stored in Petri dishes until emergence of adults. All experiments and insect rearing and storage were conducted at $22 \pm 2^{\circ} \mathrm{C}$ and $16 \mathrm{~L}: 8 \mathrm{D}$.

\subsubsection{Effect of Host Age on Suitability for Parasitism}

Labeyrie (1960) found $D$. pulchellus females to prefer the younger of two pupae when given a choice of different-aged hosts. However, the ultimate cause for this preference was not identified and it has not been clear whether there is a maximum suitable host age for parasitoid development. The survival and development time of $D$. pulchellus in hosts of different ages was measured to determine the window of opportunity for parasitism once a leek moth pupates. Four BugDorm cages were prepared, each containing approximately 100 host-experienced female $D$. pulchellus of varying ages and two vials of $20 \%$ sucrose solution with cotton wicks. The parasitoids in all cages were deprived of hosts for two days. Following this host-deprivation period, approximately 100 leek moth pupae were placed into each cage for 24 hours to allow parasitism to occur. Each cage was allocated to a different host age treatment group, such that females were presented with either 1-, 3-, 5- or 7-day-old host pupae. The parasitoids received a new cohort of host pupae each day for three days. The age of pupae assigned 
to a particular cage was held constant over the three days to minimise host rejection that could occur if hosts of significantly different quality were offered sequentially (Browne and Withers, 2002). Following parasitism, the pupae were placed into marked Petri dishes and stored at $20 \pm 1^{\circ} \mathrm{C}, 16 \mathrm{~L}: 8 \mathrm{D}$. The dishes of pupae were then checked daily to record the emergence and development time of parasitoid offspring.

\subsubsection{Effect of Parasitoid Physiological State and Test Environment on Oviposition}

Each of the seven factors tested consisted of two levels, one of which is considered to be the "ideal state" (see Table 4-1). The ideal state is the treatment level predicted, based on foraging theory and past empirical studies (e.g. Roitberg et al., 1992; Roitberg et al., 1993), to result in the greatest motivation to oviposit and, therefore, the highest rate of host mortality and progeny production. The effect of each factor on ovipositional drive was measured by comparing the number of hosts killed and progeny produced by females in the two treatments. The factors of interest were assessed in independent experiments, meaning that while the effect of one was being tested, the remaining six variables were controlled. Furthermore, the physiological states and test environments of the control groups were consistent between experiments.

Prior to these experiments, approximately 100 published accounts of non-target tests for entomophagous agents, dating back to 1984, were reviewed. A large majority of the past tests used mated, sugar-fed and naïve parasitoids in trials; therefore, these states were assigned to the control group in the current study. The age and density of parasitoids used is more variable among studies, as is the size of the test arena and whether host plant material was included. The following is a description of the preparation and testing of females assigned to the control group, against which the experimentally manipulated females were compared. Newly emerged females allocated to experiments were isolated and housed in Petri dishes ( $9 \mathrm{~cm}$ width $\times 2.5 \mathrm{~cm}$ depth) with two males for three days until the oviposition trials began. Diadromus pulchellus adults mate readily in Petri dishes and mating was often seen to occur within minutes of combining males with virgin females. Each Petri dish contained a cotton bud soaked in $20 \%$ sucrose solution and all cotton buds were refreshed every Monday, Wednesday and Friday. These females were not given access to any hosts prior to testing. A trial was started by introducing a single, 
three-day-old parasitoid into a test arena consisting of a Petri dish $(9 \mathrm{~cm}$ width $\times 2.5 \mathrm{~cm}$ depth) with a sucrose solution-infused cotton bud and 15 healthy pupae ( $<24$ hours old) interspersed evenly over the floor of the dish. The maximum oviposition rate of $D$. pulchellus is 10-12 eggs/day (Labeyrie, 1960); therefore 15 hosts were sufficient to ensure parasitoids were not host-limited. Parasitoids were allowed to oviposit in these hosts for 24 hours. At the end of the trial period, parasitoids were discarded and the pupae were stored in labelled Petri dishes to record host mortality (i.e., failure of a non-target adult to emerge due to physical damage caused by the parasitoid, complete or partial development of a parasitoid larva, or poor health of the pupa) and emergence of parasitoid offspring. The sections below explain how this procedure was modified in order to test the effects of variation in the condition of parasitoids and their foraging environment on oviposition activity.

\section{Mating Status}

Mated females were compared to virgin females. Virgin female parasitoids were prepared by keeping them in isolation from males during the three-day period between eclosion and use in the experiment. All females with males were assumed to have mated at least once; however, the effect of the number of copulation events experienced by a female was not tested.

\section{Nutritional Status}

Sugar-fed females were compared to water-fed (= sugar-starved) females. Sugarstarved parasitoids were fed only pure water from eclosion to the time of the experiment. The dental cotton buds in the pure water treatment were similarly refreshed every Monday, Wednesday and Friday. This treatment group was also only provided with pure water during the 24-hour trial period. All females survived until the end of the experiment without access to sugars. The longevity of sugar-starved females was measured by keeping these parasitoids in their Petri dishes with only pure water following the 24-hour oviposition trial until death.

Diadromus pulchellus females are occasionally seen to crawl into their hosts' cocoons following oviposition and consume minute amounts of host haemolymph that 
exudes from the oviposition wound (Rojas-Rousse, 1980). To test whether this behaviour has any effect on female longevity, a separate cohort of females was simultaneously held in Petri dishes under identical conditions as those described for females in the water-fed treatment but were not provided with any hosts. The longevity of the two groups was compared.

\section{Parasitoid Age}

Three-day-old females were compared to ten-day-old females. Parasitoids in the ten-day-old treatment were stored under the same conditions described above but for one week longer than their control group counterparts. Mortality in female parasitoids during the ten-day pre-experimental period was extremely low and no females died during the experiment. The mean longevity of $D$. pulchellus females held in Petri dishes under laboratory conditions and with continuous access to $20 \%$ sucrose solution is 43.6 days \pm 1.3 S.E $(n=85)$. Thus, the variation in ages examined here only covers the first quarter of female survival. Nonetheless, ten days without any exposure to host pupae is probably an extreme scenario of host deprivation, which is likely to influence foraging behaviour. Moreover, $D$. pulchellus longevity in the field is likely to be significantly lower.

\section{Host Experience}

Naive (= host-deprived) females were compared to experienced females. To give parasitoids a host experience, a single leek moth pupa $(<24$ hours old $)$ was placed into each Petri dish for a period of three hours, which was a sufficient duration to enable a parasitoid to encounter the pupa in its dish. This pre-trial host experience was done when parasitoids were two days old, at which time they are typically reproductively mature (Labeyrie, 1960). The 24-hour period between initial host experience and the experiment allowed females to replace any mature eggs that may have been laid during the initial experience and also provided time for any possible effects of host contact on ovigenesis and egg maturation to materialise. 
Parasitoid Density

Solitary females were compared to females foraging in groups. The effect of perceived competition was tested by running trials with three females foraging simultaneously in the same Petri dish arena. All females were contained separately during the 3-day pre-experimental period and were only combined at the start of the trial.

\section{Arena Size}

A small Petri dish arena was compared to a larger, mesh-walled cage. The Petri dish arena, used in all the other experiments, was considered to be a small test environment. The large experimental arenas were the same $30 \mathrm{~cm}^{3}$ BugDorms used for holding the $D$. pulchellus cultures. These cages were approximately 150 times more voluminous than the Petri dish arenas. The 15 leek moth pupae were spread out across the floor of the BugDorms in five rows of three. Whereas the distance between pupae in the Petri dish arenas was typically $1-2 \mathrm{~cm}$, the inter-pupal distance in the large arenas was greater than $8 \mathrm{~cm}$. Thus, the small size of these Petri dishes inevitably increased the frequency at which parasitoids encountered their hosts and food source compared to the large arena treatment.

\section{Presence of Host Plant Cues}

Females foraging in the absence of host plant material were compared to those with host plant material present. All control group trials were run in Petri dishes in the absence of any host plant material. To test whether contact with the host substrate can affect oviposition behaviour, some females were allowed to forage in the presence of damaged leek leaves. A $2 \mathrm{~cm}^{2}$ disc of dried leek leaf containing extensive feeding damage and leek moth larval frass was placed in the centre of the arena. The host pupae were distributed over the floor of the arena as in the control group.

\subsubsection{Data Analyses}

A one-way ANOVA was used to detect effects of host age on parasitoid emergence rates and development times. When significant differences were found, all pairwise comparisons were made using the Tukey Test. Two response variables were measured to 
test the effect of physiological and environmental factors on ovipositional drive: (1) the number of progeny produced and (2) the number of host pupae killed by a female over a 24 hour period. A pupa was considered killed if it failed to yield a leek moth adult. This therefore includes all pupae yielding parasitoid offspring. Realistically, host mortality could occur from physical damage caused by the parasitoid, complete or partial development of a parasitoid larva or poor health of the pupa itself. Each experimental replicate was accompanied by a negative control, consisting of a Petri dish with 15 leek moth pupae but without a parasitoid. This was to assess the quality of the leek moth hosts and to determine the proportion of pupal mortality that was caused by intrinsic factors. If the mean mortality in the negative controls exceeded $20 \%$ (three dead pupae) then the corresponding trials with parasitoids were excluded from the subsequent analysis. Pupal death occurred, but was not frequent, in the negative controls. Since the pupae used for both parasitoid treatments in each experiment were taken from the same culture at the same time, any effects of unrelated host death on the results should have been equal between treatments. For each experiment outlined above, a minimum of 30 replicates was run. The experimental results consisted of count data and while some data sets were normally distributed with equal variance, most could not be normalised through transformation. Therefore, Mann-Whitney $U$ tests were run to compare the number of progeny produced and the number of hosts killed by the two treatment groups in each experiment. The comparison of survival times of sugar-starved parasitoids with and without hosts was carried out using Student's t-test.

\subsection{Results}

\subsubsection{Effect of Host Age on Suitability for Parasitism}

Parasitoids readily attacked the leek moth pupae they were provided, regardless of host age. Emergence was highest from 1-day-old and 3-day-old hosts, declined slightly in 5-day-old hosts and then dropped drastically in 7-day-old hosts (Figure 4-1a). Host age similarly had an effect on parasitoid development time (Figure 4-1b). There was no significant difference between development time of males and females ( $t$-test: $t=1.228$, $\mathrm{P}=0.231$ ), therefore the sexes were combined for development time analysis. Again, younger hosts appeared to be of higher quality with respect to parasitoid development 
time. The time between parasitism and emergence of $D$. pulchellus adults was shortest in 1-day-old and 3-day-old hosts, slightly longer in 5-day-old hosts and significantly longer in 7-day-old hosts (Figure 4-1b).

\subsubsection{Effect of Parasitoid Physiological State and Test Environment on Oviposition}

Mating Status

Mating status had no apparent effect on the number of offspring produced $(T=$ $1121.0 ; P=0.292)$ or the number of leek moth hosts killed $(T=1157.0 ; P=0.129)$ by $D$. pulchellus females (Figure 4-2).

\section{Nutritional Status}

Parasitoid nutritional status had a significant effect on both the number of offspring produced $(T=1326.5 ; P<0.001)$ and the total number of hosts killed $(T=1332.0 ; P<$ 0.001 ). Females fed a $20 \%$ sucrose solution since emergence had higher reproductive success during the trial and therefore had a greater impact on leek moth survival than females fed only water (Figure 4-2). The longevity of females fed a water-only diet and completely deprived of hosts $(7.1 \pm 0.3$ days; $n=34)$ did not differ from that of the fernales provided with 15 hosts on the third day after eclosion $(7.7 \pm 0.3$ days; $n=33)(t$ $=-1.644 ; P=0.105)$.

Parasitoid Age

Parasitoid age influenced the number of progeny emerging from host pupae $(T=$ $2003.0 ; P<0.001)$ as well as the number of hosts killed $(T=1958.0 ; P<0.001)$. On average, 10-day-old parasitoids had lower foraging success and generated lower leek moth mortality than 3-day-old individuals (Figure 4-2).

\section{Host Experience}

Previous host experience had an inconsistent effect on parasitoid foraging. Parasitoids with prior exposure to leek moth pupae produced more offspring $(T=2862.5$; $P=0.038$ ) than naive individuals. However, there was no significant difference in the 
number of hosts killed by parasitoids in the two treatment groups $(T=2718.0 ; P=0.263)$ (Figure 4-2).

Parasitoid Density

Parasitoid density influenced both the number of progeny produced and the number of hosts killed. Although fewer offspring emerged when a single parasitoid was present, this was not significantly different than when there were three parasitoids foraging simultaneously ( $T=1637.5 ; P=0.335)$. In contrast, significantly more host pupae were killed in the aggregated parasitoid treatment $((T=1873.0 ; P<0.001)$ (Figure 4-3).

\section{Arena Size}

The size of the test arena had no impact on parasitoid oviposition (Figure 4-3). The number of progeny produced ( $T=1184.5 ; P=0.893)$ and the number of hosts killed ( $T$ $=1099.0 ; P=0.367$ ) was similar despite the vastly greater space available to parasitoids in the BugDorm cages.

Presence of Host Plant Cues

The presence of pieces of infested leek leaves did not affect the number of offspring produced $(T=1280.5 ; P=0.733)$ or the number of leek moth hosts killed $(T=1205.5 ; P$ $=0.249$ ) by $D$. pulchellus females (Figure 4-3).

\subsection{Discussion}

Past research has shown that the foraging decisions made by both herbivorous and entomophagous insects can be influenced by the physiological state or previous experiences of the forager (Bell, 1990; Lewis and Martin, 1990; Vet and Dicke, 1992; Browne, 1993). In the context of biological control, the importance of the physiological state of an agent has been discussed for some time. For instance, Vet and Dicke (1992) suggested that agents should be released when they are in a physiological state that renders them most responsive to host-related cues in order to achieve the greatest impact. However, it is only more recently that physiological state is being considered as an 
important element in host specificity testing of agents for arthropod biological control. The aim of this study was to test the concept that a specific change in a physiological or informational state could have a predictable effect on the likelihood of oviposition by a parasitoid in its common host. The "killing power" (host mortality and offspring production) of $D$. pulchellus was quantified as a measure of motivation to oviposit since over $70 \%$ of attacks result in host mortality (Rojas-Rouse, 1980) and females tend to reattack their hosts under prolonged exposure (Kalmes, 1984), increasing the likelihood of mortality. These response variables are considered to be better indicators of ovipositional drive than other proxies, such as search efficiency or patch time allocation, and are considered more meaningful in the context of non-target testing since an oviposition event is the ultimate indicator of host acceptance. In each experiment, a single factor was manipulated and the degree of host exploitation by $D$. pulchellus females was compared to that of individuals from a control group (see Table 4-1 for descriptions).

Of the seven variables tested here, four were found to have a measurable effect on oviposition. The three factors not showing any effect on host mortality or progeny production were mating status, cage size and presence/absence of the host plant. A number of previous studies found mating to affect parasitoid behaviours ranging from locomotor activity to oviposition frequency (e.g., Michaud and Mackauer, 1995; Para et al., 1996; Pompanon et al., 1999). In all of these cases, mated individuals exhibited increased activity and an enhanced readiness to oviposit. Moreover, Labeyrie (1959) showed that virgin $D$. pulchellus females have a significantly reduced fecundity compared to mated females. In spite of these physiological differences, in the current study the number of hosts killed and progeny produced by mated and unmated females over 24 hours was not affected.

Smaller containers were expected to enhance motivation to oviposition through an increase in the frequency of host encounters; however, proximity to hosts did not affect parasitism rates. Although contact with host cocoons normally elicits a strong oviposition response in D. pulchellus (Bekkaoui and Thibout, 1992), it is possible that desensitisation occurs after a period of time. Such loss of stimulus could result from exhaustion of egg or energy resources or from an increased frequency of encounters with previously 
parasitised hosts. Thus, arena size may have a more detectable effect in tests where the agent is not saturated with hosts.

Parasitoids are generally assumed to use plant-derived volatile chemical stimuli to locate the host habitat. Once a host feeding site has been discovered, host-derived, often non-volatile, cues play a larger role in host location and recognition (Vet and Dicke, 1992). Nevertheless, it is possible that parasitoids respond to more than just non-volatile contact stimuli in the final stage of the search process. For example, Mattiacci et al. (2000) demonstrated that parasitoids exposed to host plant odours produced nearly twice as many progeny as individuals without the odour treatment. For this reason, it was predicted that $D$. pulchellus females would show enhanced oviposition activity in the presence of host-damaged leek leaves. However, there were no significant differences in host mortality or parasitoid offspring production under the two treatments. It is possible that parasitoid locomotor activity was enhanced in the presence of the damaged host plant, as described by Lecomte and Thibout (1983), but this did not translate to greater host exploitation. While egg production in $D$. pulchellus is stimulated by contact with leek moth pupae (Labeyrie, 1964), there is no record of the host plant affecting parasitoid fecundity.

Parasitoids may display enhanced oviposition activity due to a perceived reduction in life expectancy (e.g., Roitberg et al., 1992, 1993; Fletcher et al., 1994). Food deprivation is one method of modifying parasitoid behaviour through a change in the perceived longevity; however, Lee and Heimpel (2007) showed that nutritional status may not always affect the degree of host exploitation despite influencing other behavioural parameters like patch time allocation. The longevity of D. pulchellus females fed a sucrose solution was approximately six times longer than that of water-fed females. Although all the water-fed parasitoids survived the full experimental process, this treatment was likely very effective in creating a perception of reduced longevity. Parasitoids were occasionally observed to host-feed during the foraging trials; however, this has previously been shown not to affect longevity or fecundity in $D$. pulchellus (Labeyrie, 1960). The results obtained here further demonstrated that water-fed females provided with an opportunity to host-feed for 24 hours did not live longer than those completely deprived of hosts. 
Despite their reduced longevity, the water-fed $D$. pulchellus females did not display enhanced oviposition activity. Instead, in contradiction to our expectations, sucrose-fed females produced twice as many offspring during the trial period as water-fed females. A possible explanation for the poorer performance of water-fed parasitoids is that host handling times and rest periods were increased due to limited energy reserves. Alternatively, water-fed females may have resorbed mature eggs in a trade-off between reproductive capacity and energy conservation (Labeyrie, 1960). The reduced egg load could then cause a decrease in motivation to oviposit (Minkenberg et al., 1992). This is especially plausible since the parasitoids in this experiment were not given access to any hosts during the three-day pre-trial period and the perception of low host abundance may increase the likelihood of egg resorption (Santolamazza-Carbone et al., 2008). Hence, for species capable of egg resorption, a pre-trial starvation treatment may actually create the undesirable effect of reduced parasitism despite creating the perception of reduced life expectancy.

Parasitoid age has been shown to affect several facets of foraging behaviour such as responsiveness to odours (Drost et al., 1988; Para et al., 1996), patch residence time (Goubault et al., 2005), oviposition rate (Völkl and Mackauer, 1990; Michaud and Mackauer, 1995) and host instar preference (Weisser, 1994). Despite the number of examples demonstrating the importance of age, the specific mechanisms behind variable behaviour are often not elucidated. Age is inherently correlated with a number of biological parameters including experience, nutrition, perceived longevity and egg load and the effects of these factors are often not easily separated. Nonetheless, parasitoid age is an easily-manipulated attribute and should be taken into consideration when studying behaviour as it can have profound consequences on foraging decisions.

Parasitoids are generally expected to express an increased readiness to oviposit as they become older (Withers and Browne, 2004). This may be due to a combination of factors, the two most important of which seem to be increased egg load and reduced life expectancy. Withers and Browne (2004) caution that age may have the opposite effect on strictly pro-ovigenic species, since these parasitoids can only experience a decrease in egg load, either through oviposition experience or resorption of the eggs. The current study, however, shows that increased age in a synovigenic parasitoid can also lead to an 
apparent decrease in motivation to oviposit. Ten-day-old $D$. pulchellus females produced approximately half as many offspring as 3-day-old adults while total host mortality was similarly low in the older age treatment. Labeyrie (1960) stated that when D. pulchellus is provided with hosts each day following emergence the daily fecundity of both mated and virgin females peaks by day 9 , but females can remain reproductively active for up to five weeks. When Labeyrie (1960) deprived parasitoids of hosts for five or ten days, he found no substantial difference in the frequency of oviposition by 6- and 11-day-old $D$. pulchellus. The strong age effect observed in the current study may have been due to the specific ages used, which differed slightly from those of Labeyrie (1960). It must also be noted here that the current study measured oviposition activity in terms of host mortality and progeny production when an abundance of hosts were provided to parasitoids, whereas Labeyrie (1960) counted the number of eggs laid into a single host over a 24hour period. It would not be surprising if Labeyrie's parasitoids responded differently under such conditions given the ability of $D$. pulchellus to discriminate between healthy and parasitised hosts (Labeyrie, 1960) and the fitness costs of self-superparasitism (van Lenteren, 1981).

Diadromus pulchellus will resorb eggs in response to either low energy reserves or low host availability. Although Labeyrie (1960) found that 10-day-old, host-deprived females tended to have more eggs in the process of resorption than 3-day-old, hostdeprived individuals, the older parasitoids still possessed more mature eggs on average. Thus, the lower reproductive output of 10-day-old parasitoids in the current experiment should not be due to a relatively lower egg load. In light of the available data, it is not possible to conclude why the older females in this case exhibited lower host exploitation than their younger siblings. The relationship between young and old parasitoids could change if different ages are compared, since $D$. pulchellus fecundity begins to decline steadily after around 10 days of age (Labeyrie, 1960). The ages of 3 and 10 were chosen for this experiment as they are both realistic and practical in the context of non-target testing and, as mentioned, fall within the known period of maximum daily fecundity. Although the younger parasitoids demonstrated the greatest motivation to oviposit, the use of even younger (1- or 2-day-old) parasitoids is not recommended since many of these individuals do not yet contain mature eggs (Labeyrie, 1960). 
A parasitoid's foraging decisions can be strongly influenced by previous experiences it has had. It is because of this continuous learning that care must be taken to ensure that parasitoids are subjected to identical conditions prior to experimentation. Parasitoid learning can occur during pre-imaginal development, at emergence of the adult or while searching for hosts, and it is thought to be an important component for increasing search efficiency (Godfray, 1994). Adult learning typically takes the form of conditioning, whereby responses to stimuli are enhanced through their association with a reinforcing stimulus, such as oviposition in a suitable host (Vet and Dicke, 1992). Due to the nature of associative learning with positive reinforcement, these experiences tend to result in enhanced responsiveness when parasitoids are later exposed to the same stimulus. Thus, there is strong agreement among past studies that experience with hosts or host-related materials will lead to more direct flight towards those odour sources in wind tunnels (e.g., Pettit et al., 1992; Para et al., 1996; Fujiwara et al., 2000). Furthermore, experienced parasitoids have a greater tendency to oviposit in or on the associated hosts (e.g., Kaiser et al., 1989; Ambriz et al., 1996; Kerguelen and Cardé, 1996; Bjorksten and Hoffmann, 1998). It is therefore not surprising that D. pulchellus females that had been given a host pupa one day prior to the host exploitation trials showed higher motivation to oviposit than naïve females. Although a previous host experience did not have as strong of an effect as parasitoid nutritional status or age did, it nonetheless demonstrates a measurable change in behaviour.

When the pupae used in giving $D$. pulchellus their pre-trial host experience were reared out, the majority of them yielded healthy leek moth adults. This means that parasitism either failed ("pseudoparasitism") or never occurred at all. Naïve D. pulchellus females will often stand motionless over a host cocoon for long periods of time before attempting to oviposit. It is possible that the three-hour period for a host experience was too brief to ensure that hosts were successfully parasitised. Regardless of the outcome of the pre-trial host exposures, the experience is still regarded as an important learning event since contact with hosts stimulates oocyte production in D. pulchellus (Labeyrie, 1964). Additionally, Lecomte and Thibout (1993) demonstrated that parasitoid contact with frass and/or host pupae for durations from 10 minutes to 5 hours and with a latency period of 10 minutes to 48 hours, had a strong effect on odour response. After host contact, 
experienced D. pulchellus females walked more directly to odour sources. Since the degree of host contact prior to testing can influence the strength of the effect (Kergeulen and Cardé, 1996), it is possible that a larger difference in motivation to oviposit would have been observed if $D$. pulchellus had been given more time with hosts in the pre-trial experience.

The final factor found to affect $D$. pulchellus oviposition activity was parasitoid aggregation during foraging. Withers and Browne (2004) recommended that potential biological control agents be tested in groups when used in host specificity trials since the perceived competition could enhance their foraging effort and motivation to oviposit. For instance, Visser et al. (1990) found that parasitoids foraging under competition spent longer in host patches and were more likely to superparasitise hosts than those foraging alone. Carbone and Rivera (2003) later showed that competition caused parasitoids to lay significantly more eggs. However, this effect of competition on foraging motivation is not universal. Goubault et al. (2005) observed that parasitoids spent less time in patches with increasing numbers of competitors and that the physiological and informational state partly determined which individuals would stay or remain. In the current experiment, a solitary parasitoid was able to produce approximately as many healthy offspring as three females combined. And although significantly more leek moth pupae were killed in the aggregated parasitoid treatment, this value was not three times higher than in the solitary parasitoid control, as would be expected if parasitoid-induced mortality were additive. At first glance, it appears that parasitoid motivation to oviposit was reduced by the perception of competition. However, two pieces of information suggest an alternative explanation. First, there was occasional competition for hosts in the form of chasing, biting and even stinging one another. These aggressive interactions occurred despite an abundance of hosts, all of which were of a similar age $(<24$ hours old). Second, Labeyrie (1960) observed that $D$. pulchellus females conspicuously concentrate their eggs in certain hosts when given a selection. These two pieces of evidence imply that groups of these parasitoids will less efficiently exploit a host patch because they do not distribute their oviposition efforts evenly over the available hosts. If this latter explanation is valid, it means that the current test cannot quantify the contribution of each parasitoid to host mortality. An alternative dependent variable, such as the number of oviposition events 
per unit time, would be required to effectively test the impact of parasitoid density on oviposition behaviour.

In conclusion, variation in the physiological and informational state of $D$. pulchellus females does affect their oviposition behaviour, expressed as changes in host mortality and offspring production. Parasitoid age and nutritional status had the greatest impact while prior host experience and intraspecific competition each only influenced one of the two response variables. Most surprising was the direction of the effect of most of these factors. Whereas Withers and Browne (2004) predicted that increased age, pre-trial starvation and perceived competition should increase a parasitoid's motivation to oviposit, these factors had the opposite effect for $D$. pulchellus. Thus, while the proposal by Withers and Browne (2004) is very insightful, it should be cautioned that the specific effects of physiological and environmental factors may be less universal than assumed. Rather, the manipulation of specific parasitoid states prior to non-target testing may require previous knowledge of how changes in those states affect foraging behaviour. Bai and Mackauer (1991) demonstrated this point by showing that different parasitoid species vary in their response to the same variables. As a result, the extent to which biological control practitioners will control for "ideal parasitoid state" may depend largely on what is known of the biology of the agent.

A parasitoid's foraging decisions could be influenced by many more physiological and environmental factors than were tested here. Hence, there are still many aspects of. motivation to oviposit and host range expression to explore. It seems very likely that the apparent effect of physiological or informational state could also vary depending on which behavioural parameters are measured. The response variables used here (number of hosts killed and parasitoid offspring produced) should be highly correlated for a parasitoid agent that does not exhibit destructive host feeding. As expected, the results are very consistent between Figures $4-2 a$ and $4-2 b$ and between Figures 4-3a and 4-3b. Nevertheless, significant differences in oviposition activity were not always consistent between the two dependent variables. From this study it is not possible to determine whether measuring host mortality or offspring production is more effective for detecting differences in an agent's motivation to oviposit. 
An important study would be to directly compare the direction and magnitude of effects that factors have on different facets of behaviour. If the effects tended to be consistent across several behavioural parameters, then it would be easier to confidently identify the ideal state that an agent should be in for non-target testing. A simple but important point to be made is that the behavioural attribute used for determining an agent's ideal state prior to host range testing should be relevant to the planned host range test design. For host range tests exploring an agent's attraction to non-target habitat (e.g., Yong et al., 2007), behaviours associated with responsiveness to chemical and visual cues are most appropriate (e.g., wind tunnel flight response or locomotor activity). In contrast, oviposition-related behaviours (e.g., time until attack, likelihood of host acceptance or degree of attack) are most meaningful for tests observing whether an agent will attack non-target hosts. 
Table 4-1. List of factors tested for their effects on the motivation of Diadromus pulchellus Wesmael to oviposit into its leek moth hosts. Each factor consists of two levels, one of which is considered to be the "ideal state" ("State 1" column). The ideal state is the treatment level predicted, based on foraging theory and empirical studies, to result in the greatest motivation to oviposit. Therefore, "State 1" treatments are expected to show higher rates of host morality and offspring production than the "State 2" treatments.

\begin{tabular}{clll}
\hline Factor Type & Factor & State 1 & State 2 \\
\hline \multirow{2}{*}{$\begin{array}{c}\text { condition of } \\
\text { parasitoid }\end{array}$} & mating status & mated * & virgin \\
& age & fed pure water & fed sugar-water * \\
& host experience & 10 days & 3 days * \\
\hline \multirow{2}{c}{$\begin{array}{c}\text { experienced } \\
\text { environment }\end{array}$} & parasitoid density & 3 females & 1 female * \\
& arena size & small * & large \\
\hline
\end{tabular}

* indicates the state of each variable used in the control group 

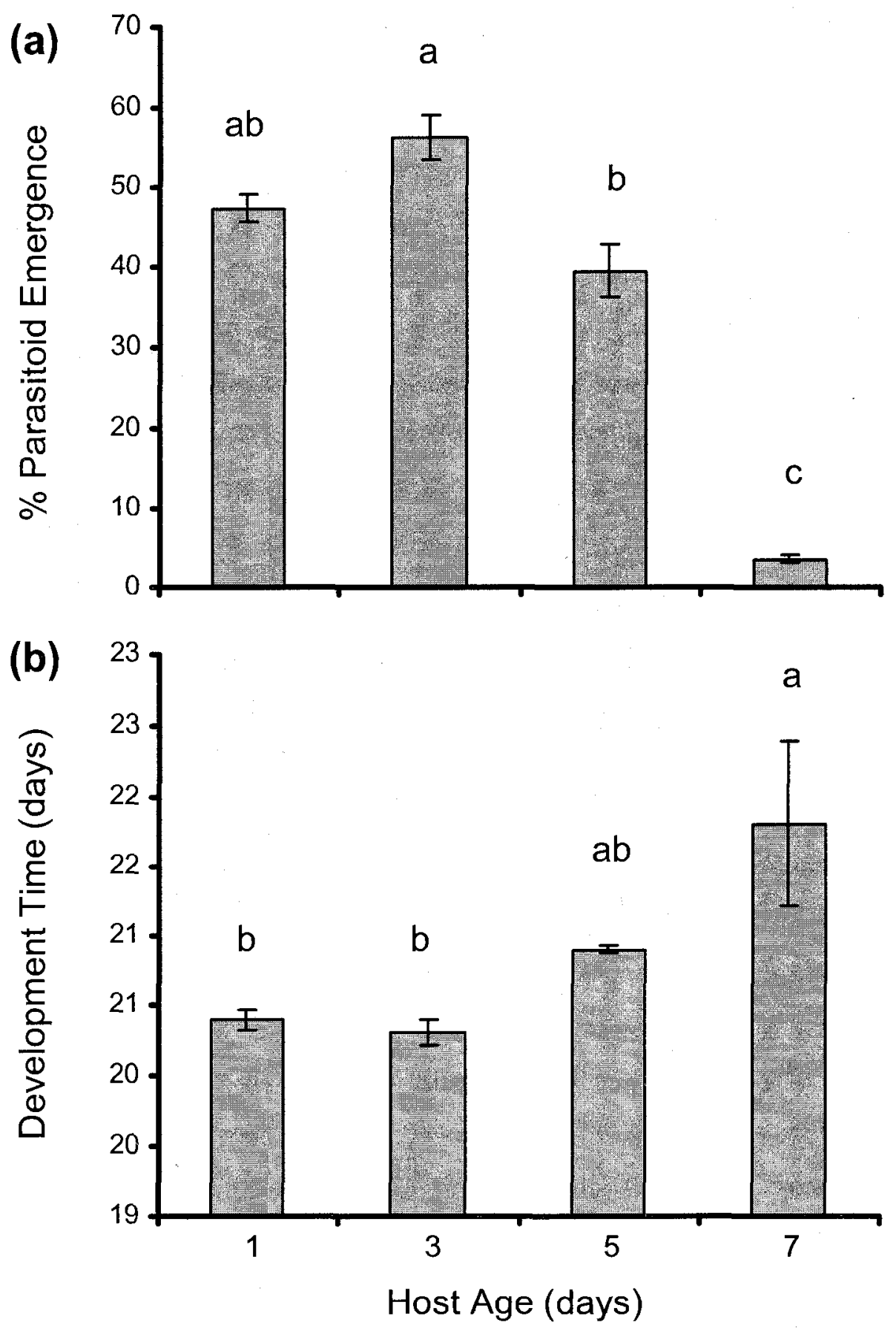

Figure 4-1. Effect of host age on (a) emergence rates $(F=100.985 ; P<0.001)$ and (b) development times $(F=6.037 ; P<0.001)$ of Diadromus pulchellus Wesmael. 

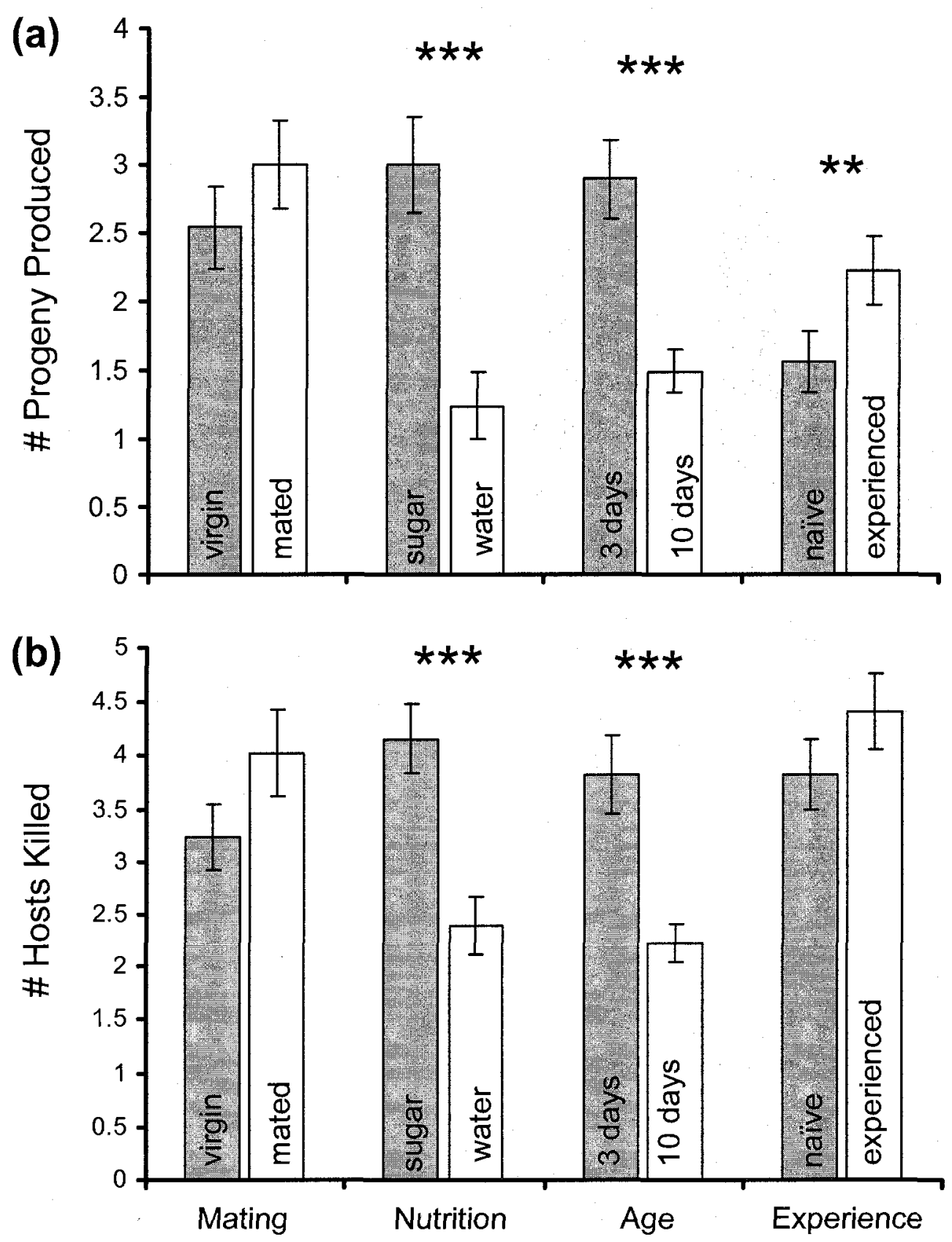

Figure 4-2. Number of (a) offspring produced and (b) hosts killed by Diadromus pulchellus Wesmael females under varying physiological states (mating: virgin $(\mathrm{n}=35)$, mated $(\mathrm{n}=31)$; nutrition: sucrose $(\mathrm{n}=32)$, water $(\mathrm{n}=33)$; age: 3 -day-old $(\mathrm{n}=38), 10$ day-old $(n=45)$; experience: naïve $(n=54)$, experienced $(n=49))$. Treatments with white bars were predicted to result in greatest motivation to oviposit. $* * P<0.01 ; * * * P$ $<0.001$ 

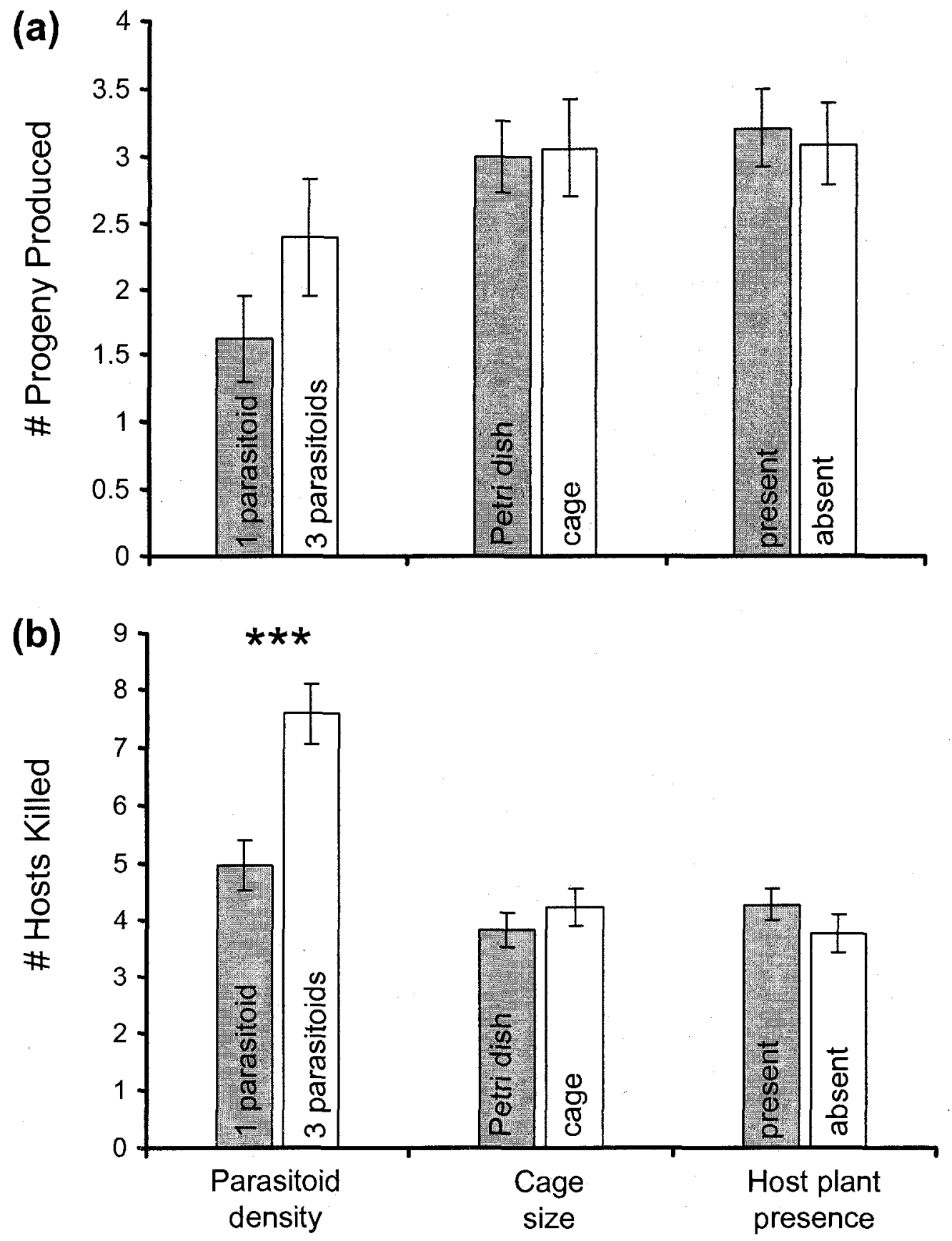

Figure 4-3. Number of (a) offspring produced and (b) hosts killed by Diadromus pulchellus Wesmael females under varying test environment conditions (parasitoid density: single parasitoid $(n=39)$, three parasitoids $(n=39)$; cage size: Petri dish $(n=$ 34), BugDorm cage $(n=34)$; host plant presence: present $(n=35)$, absent $(n=39))$. The values shown for the high parasitoid density treatment are totals from all three parasitoids. Treatments with white bars were predicted to result in greatest motivation to oviposit. ${ }^{* * *} P<0.001$ 


\section{Does Parasitoid State Affect Host Range Expression? A Test}

\subsection{Introduction}

Biological control practitioners are frequently faced with the daunting task of estimating an agent's ecological host range (= the set of species used for reproduction in the field (sensu Nechols et al, 1992; Onstad and McManus, 1996)). This is necessary to determine whether the agent might pose an unacceptable risk to non-target species or ecosystems. Without reliable tests, it is possible that "safe" agents could be rejected unnecessarily or that released agents could affect non-target populations (Browne and Withers, 2002). While rigorous field surveys can be carried out in the agent's native range to assess ecological host range directly (e.g., Haye et al., 2005), a more common approach is to employ laboratory-based trials to measure the agent's fundamental host range ( $=$ the set of all species that can support development of the agent (sensu Nechols et al, 1992; Onstad and McManus, 1996)). Since laboratory tests typically exclude the initial steps of the host search process, they may embellish the ecological host range of an agent (Keller, 1999, Kidd and Jervis, 2005). However, Withers and Browne (2004) argue that it is desirable to maximise the probability that non-target species will be attacked during laboratory testing in order to minimise the risk of false-negative results (i.e., results that incorrectly identify a non-target as unsuitable (sensu Marohasy, 1998)).

Parasitoid host range tests are designed to measure the response of the agent to a non-target host, usually in the form of acceptance for oviposition, and the suitability of the host for parasitoid development. However, insect oviposition behaviour can be influenced by a number of seemingly subtle factors. For instance, endogenous factors can cause variability in the behavioural responses of different individuals, or a single individual over time, despite subjecting them to identical environmental conditions (Papaj and Rausher, 1983; Browne, 1993). Similarly, changes in the environmental conditions (e.g., light, temperature, crowding) can affect parasitoid behaviour. This variability can increase the likelihood of obtaining false-negative results (Roitberg, 2000) and therefore necessitates extra test replication. On the other hand, Withers and Browne (2004) suggested using this characteristic to one's advantage by manipulating the 
condition of parasitoids so that they exhibit maximum host range expression. These authors recommended controlling the physiological and informational state of agents, as well as the rearing and test environment, to increase the agents" "motivation to oviposit". The premise behind this concept is that parasitoids with enhanced readiness to oviposit should be more willing to accept unfamiliar hosts, thereby reducing the risk of a type II error (false-negative).

As explained in the previous chapter, the guidelines spelled out by Withers and Browne (2004) on how to augment an agent's oviposition motivation are based on a set of implicit assumptions. This is because nearly all of the previous studies on identifying factors that affect motivation to oviposit have been conducted on parasitoids' common, or preferred, hosts. Furthermore, many of these past studies did not measure oviposition behaviour per se, but instead measured response variables like responsiveness to odours and search activity. The preceding chapter discussed the first two assumptions: (1) a change in physiological state that modifies one behavioural parameter, such as responsiveness to odours, would also induce a change in the parasitoid's motivation to oviposit and (2) the direction and magnitude of change in one behavioural parameter will translate to a roughly equivalent transition in the probability of an oviposition event occurring. That chapter examined whether parasitoid motivation was affected by specific changes in physiological and environmental state in a predictable way as suggested by Withers and Browne (2004). The third important assumption underlying their recommendations is that an increased readiness to oviposit in the target host will translate to a similar increase in willingness to oviposit in non-target hosts. As with the first two cases, the third assumption may not apply universally to all endogenous factors and all species.

This chapter tackles the question of whether it is possible to manipulate a biological control agent or its environment to achieve greater host range expression. These questions were addressed again using the parasitoid-host system consisting of leek moth, Acrolepiopsis assectella (Zeller) (Lepidoptera: Acrolepiidae), and its pupal parasitoid, Diadromus pulchellus Wesmael (Hymenoptera: Ichneumonidae). Diadromus pulchellus is generally considered to be a specialist on leek moth (e.g., Bekkaoui and Thibout, 1993; Lecomte and Thibout, 1993; Gauthier et al., 2004). It is well known that D. pulchellus is 
stimulated and attracted by sulphur volatiles emitted from Allium plants as well as the frass of leek moth larvae (Lecomte and Thibout, 1983; Thibout et al., 1988). While the sulphur-dominated chemical profile is a classic characteristic of Allium spp., it is also a trademark of the Brassicaceae (Bailey et al., 1961), which contains many cultivated and weedy species common in North America. Furthermore, Lecomte and Thibout (1984) showed that $D$. pulchellus is stimulated by volatile sulphur compounds from brassicaceous plants and Thibout (1988) has already demonstrated that the parasitoids can develop in Plutella xylostella (Linnaeus) (Lepidoptera: Plutellidae), which feeds on several Brassica species.

In the previous chapter, 3-day-old parasitoids were shown to produce more offspring and therefore kill more leek moth hosts than 10-day-old siblings. According to Withers and Browne's (2004) predictions, younger females would therefore be expected to express a broader host range through more frequent attacks on non-target species. The objective of this study was to conduct a thorough series of host specificity trials with $D$. pulchellus to delineate the boundaries of its host range while assessing how parasitoid physiological state can influence the outcome of such tests. In addition, both no-choice and choice assays were run to examine how the test design can affect host specificity test results.

\subsection{Materials and Methods}

\subsubsection{Selection of Non-target Species}

The test list for assessing the host specificity of $D$. pulchellus is shown in Table 5-1. Each of the species included in the list was tested because it fit at least one of the following selection criteria: (1) phylogenetic affinity to target pest, (2) ecological similarity to target, (3), safeguard species, (4) morphological similarity to target and (5) known host of another Diadromus parasitoid.

Non-targets were considered to have phylogenetic affinity to leek moth if they belonged to the same superfamily, Yponomeutoidea. Although the phylogenetic relationships within Yponomeutoidea are not well understood, those species within the family Plutellidae appear to be closely related taxonomically to the acrolepiids (Dugdale et al., 1998). Based on earlier indications that D. pulchellus was fairly host specific 
(Thibout, 1988), all non-targets outside of Yponomeutoidea were considered phylogenetic outgroups and therefore much less likely to be suitable for parasitoid development.

With respect to the ecological similarity criterion, any non-target species occurring on Allium plant hosts would be at high risk since there is a high likelihood of frequent encounter by foraging parasitoids. In eastern North America, there are perhaps a few polyphagous species of Noctuidae which occasionally feed on Allium plants, however no Microlepidoptera are known to do so (J.-F. Landry, personal communication). Therefore, there is little risk of suitable non-target species occurring on the same host plants as leek moth. A second concern is whether there are any non-target host species developing on plants that are outside the Allium genus but which possess traits, such as the Brassicaceae, that attract $D$. pulchellus females. Finally, no moths in the superfamily Yponomeutoidea in eastern North America are considered to provide an essential economic or ecological service (J.-F. Landry, personal communication).

\subsubsection{Rearing of $A$. assectella}

Leek moths were reared on mature, potted leeks in wooden cages with nylon mesh walls for ventilation. All adult moths were held in a large, well ventilated, cage and readily oviposited onto segments of leek leaves that were hung from the cage walls. Newly laid eggs were placed onto fresh leeks every 3-5 days to ensure a continuous supply of host material for parasitoids. Each day, all newly formed cocoons containing pre-pupae were collected from the rearing cages and stored for one day to allow pupation to take place. This collection system ensured that the precise age of all hosts was known. The leek moth pupae were then designated to be used as controls in non-target tests, as material for leek moth mass rearing or as host material for $D$. pulchellus mass rearing. All experiments and insect rearing and storage were conducted at $22 \pm 2^{\circ} \mathrm{C}, 16 \mathrm{~L}: 8 \mathrm{D}$.

\subsubsection{Rearing of Non-targets}

The non-targets tested with $D$. pulchellus were obtained from different sources. Four species were acquired from continuous rearing at commercial insectaries or research facilities as follows: Lobesia botrana (Denis and Schiffermüller) from Agroscope 
Changins-Wädenswil (Nyon, Switzerland), Spodoptera exigua (Hübner) from Bio-Serv (Frenchtown, NJ, USA), and Plutella xylostella (Linnaeus) and Mamestra configurata Walker from the Eastern Cereal and Oilseed Research Centre, Agriculture and Agri-Food Canada (Ottawa, ON, Canada). Temporary cultures of the remaining eight species were established using field-collected larvae of Plutella porrectella (Linnaeus), Acrolepiopsis incertella (Chambers), Ypsolopha dentella (Fabricius), Yponomeuta padella (Linnaeus), Yponomeuta cagnagella (Hübner) and adults of Plodia interpunctella (Hübner) and Pieris rapae (Linnaeus). All non-target species were reared in the laboratory on natural host plant material (see Table 5-1), with the exception of $P$. interpunctella, which was reared on a mixture of flour, almonds and rice. The cultures were monitored daily to collect all newly formed pupae for immediate use in host specificity trials.

If non-targets taken from the field are used directly in host specificity tests, there is a chance that they are already parasitised or infected with a pathogen. Such infection can affect the quality of these hosts and therefore alter parasitoid response to them (van Lenteren et al., 2006). Ideally, non-target species would be reared for a full generation in the laboratory to guarantee that all specimens are healthy prior to exposure to the agent. Seven of the non-target species tested here were reared for at least one full generation in the laboratory prior to testing. The remaining five species, $A$. incertella, A. mouffetella, $Y$. cagnagella, $Y$. padella and $Y$. dentella, were collected as larvae from the field, reared to pupation in the laboratory and used directly in trials once they had pupated. For these latter species, the inclusion of negative controls (to detect whether non-target death occurred in the absence of the agent and was therefore caused by another factor) was particularly important. Field parasitism was detected in four of these five non-targets (no parasitism in $Y$. dentella), however it always resulted in host death prior to pupation. The non-targets surviving to the pupal stage had very high survival with no signs of parasitism and were thus deemed fit for testing with $D$. pulchellus.

\subsubsection{Rearing of D. pulchellus}

A laboratory culture of $D$. pulchellus was established using parasitoids reared from leek moth pupae collected in organic and integrated production leek fields in west-central Switzerland in 2005. Fresh field-collected material was added each summer afterwards. 
To maintain the culture and ensure a continuous supply of parasitoids, a portion of $D$. pulchellus adults were housed in $30 \mathrm{~cm}^{3}$ plastic cages (BugDorm-1; BioQuip ${ }^{\circledR}$, Rancho Domingo, CA, USA) and provided with a $20 \%$ sucrose solution. These parasitoids were provided daily with fresh leek moth hosts, which, once parasitised, were stored in Petri dishes for approximately two weeks and monitored for adult emergence. Newly emerging females allocated to experiments were separated and housed in Petri dishes $(9 \mathrm{~cm}$ width $\mathrm{x}$ $2.5 \mathrm{~cm}$ depth) with two males until the non-target trials began. Since sugar-fed females have higher oviposition rates (see previous chapter), each Petri dish also contained a cotton bud soaked in $20 \%$ sucrose solution and all cotton buds were refreshed every second day. Each parasitoid was randomly assigned to the "young" or "old" treatment and then held in their Petri dishes for 3 or 10 days, respectively, prior to testing.

The previous chapter also demonstrated that previous host experience can affect subsequent interactions between $D$. pulchellus and its hosts. In host specificity testing, there is a fear that agents will show a bias towards the target or rearing host due to pre- or post-emergence conditioning (Withers and Browne, 2004). To minimise the risk of associative learning prior to testing, only naïve parasitoids were used in the non-target trials. All parasitoids emerged from their hosts in the absence of plant material and were stored in clean Petri dishes with no access to hosts. To further reduce the chance of an experience-induced bias for the rearing host's food plant, all non-target and leek moth hosts in the Petri dish no-choice and choice tests were presented, in their cocoons when applicable, on an inert surface without the host plant (Withers and Browne, 2004).

\subsubsection{Simultaneous, No-choice, Black-box Test}

All 12 non-target species were exposed to $D$. pulchellus females in small arena, nochoice tests. These were also black-box trials (sensu van Lenteren et al., 2006) in that the interaction between parasitoid and host was not closely monitored, the primary objective being to measure non-target mortality and parasitoid offspring emergence. Trials were, however, occasionally observed for a short period after introduction of the agents to verify that they were actively searching for hosts (van Lenteren et al., 2006). For each non-target trial, a single, mated, naïve $D$. pulchellus female was released into a clean Petri dish $(9 \mathrm{~cm} \times 2 \mathrm{~cm})$ containing a single, one-day-old non-target pupa and a cotton 
wick soaked with a $20 \%$ sucrose solution. A positive and negative control was run simultaneously for each non-target trial. Positive controls (to ensure that lack of attack is not due to problems with parasitoid quality or unfavourable conditions) were run by releasing another mated parasitoid of the same age into a second Petri dish with a oneday-old pupa of the target, leek moth. Negative controls consisted of a non-target pupa prepared in the same way as described above but with no parasitoid added to the arena. All hosts were presented to parasitoids in the absence of their host plant material; however, as the hosts had not been washed, they likely carried the scent of their host plants. The experiment was run for 24 hours to provide sufficient time for the parasitoids to attack the pupae in their Petri dishes and to control for a possible "time-of-day effect". This long exposure time should also increase the likelihood of non-target acceptance due to the progressive decrease in acceptance threshold that occurs during a period of host deprivation (Browne and Withers, 2002).

For every non-target species, a minimum of 100 replicates were run, 50 with threeday-old parasitoid females and 50 with ten-day-old females. Following the trials, the pupae were stored in labelled Petri dishes. Attack rates on each non-target species and the leek moth controls were estimated based on rates of parasitoid and moth emergence as well as the incidence of premature death of the host pupae. The specific response variables analysed were host mortality (i.e., failure of a non-target adult to emerge due to physical damage caused by the parasitoid, complete or partial development of a parasitoid larva, or poor health of the pupa) and successful production of parasitoid progeny in the non-target and target hosts. A non-target was classified as "acceptable" for oviposition if the number of dead pupae was greater in the parasitoid treatments than the negative controls. A species was considered "suitable" if the agent was able to complete development to the adult stage. The species $P$. xylostella, $P$. porrectella, A incertella, $Y$. dentella, A. mouffetella, S. exigua and $M$. configurata were tested at the National Arthropod Containment Facility (NACF) in Ottawa, Canada while Y. padella, $Y$. cagnagella, $L$. botrana, $P$. interpunctella and $P$. rapae were tested at CABI EuropeSwitzerland in Delémont, Switzerland. 


\subsubsection{Choice Tests Between Acrolepiopsis assectella and Plutella xylostella}

Since the no-choice tests described in the previous section demonstrated that $P$. xylostella was a frequently attacked and suitable host for D. pulchellus, a series of choice tests of increasing complexity was then run to evaluate whether parasitoids still attack the non-target in the presence of leek moth and to explore how the experimental design can affect the agent's acceptance of this non-target species. Once again, the results are compared from trials in which 3-and 10-day-old females were used.

\section{Direct Choice Test}

A simple initial choice test was set up where a single leek moth pupa was placed in close proximity to a $P$. xylostella pupa in a plastic vial $(3 \mathrm{~cm}$ diameter $\mathrm{x} 10 \mathrm{~cm}$ height). Fine (000) insect pins were used to secure both cocoons to a foam plug, placed at the base of the vial. The two hosts were pinned side-by-side, so that their cocoons were nearly touching, and at the centre of the foam plug so that neither pupa was next to the vial wall. The objective of this test was to measure the frequency of attacks on the target and nontarget hosts when the agent is able to detect both simultaneously (a true choice test). A single, mated, naïve parasitoid aged 3 or 10 days was introduced into the vial, which was sealed with a second sponge plug. This relatively small space helped guarantee that the parasitoids found the hosts after only a few moments of searching. Each parasitoid was observed until it had discovered the pupae and made a "choice", defined as showing oviposition behaviour (bending of abdomen) or coming to rest on one host for $>1$ minute. These two behaviours only occurred following a period of cocoon inspection. Each trial was terminated once a choice had been made and, in all cases, this occurred within 5 minutes.

\section{Petri Dish Choice Test $(1: 1)$}

Two pupae - one leek moth and one P. xylostella - were placed in a Petri dish $(9 \mathrm{~cm}$ x $2 \mathrm{~cm}$ ) along with a cotton bud soaked with $20 \%$ sucrose solution. In this experiment, the pupae were placed approximately $5 \mathrm{~cm}$ apart, always $>1 \mathrm{~cm}$ away from the edge of the arena, and were anchored in place by pinning their cocoons to a small piece of filter paper. The weight of the pin and paper prevented the D. pulchellus from dragging the 
cocoon across the Petri dish during oviposition attempts, as often happens with unanchored hosts. The first aim of this test was to observe how often parasitoids chose $P$. xylostella when there was a leek moth host available. As in the previous experiment, a single parasitoid was released into the arena and observed until a choice had been made. Unlike the previous experiment however, the trial was run for 24 hours. Following the trial, the pupae were stored in labelled Petri dishes and monitored to record host mortality and parasitoid emergence.

\section{Petri Dish Choice Test $(1: 4)$}

Four pupae (one leek moth and three $P$. xylostella) were placed in a Petri dish as described in the preceding experiment. For this test, the pupae were randomly spread across the floor of the arena, spaced at least $2 \mathrm{~cm}$ apart and away from the dish edge. This experiment was a test of the prediction by Withers and Browne (2004) that presenting greater numbers of non-target hosts in choice tests could lead to increased acceptance rates of those species. Again, a single agent was introduced into the arena and observed as described above. This test was also run for 24 hours to obtain data on the target and non-target mortality induced by $D$. pulchellus.

\section{Cage Choice Test}

A final choice test was conducted using leek moth and $P$. xylostella pupae attached to sections of their host plants in a larger arena (BioQuip BugDorm-1). Leek moth hosts were pinned to $15 \mathrm{~cm}$-long leaves cut from an uninfested leek plant while the $P$. xylostella pupae were pinned to small sprigs $(12-20 \mathrm{~cm}$ length) of uninfested canola. The cut ends of the leek leaves and canola sprigs were placed into a small vial of water. Two test arrangements were prepared: (1) two leek moth and two P. xylostella pupae (one pupa per sprig) and (2) four P. xylostella pupae (two pupae per sprig). Each of these host arrangements was tested with both 3- and 10-day-old parasitoids. A negative control group was set up with two leek moth and four P. xylostella pupae (two pupae per sprig), to which no parasitoids were added. This experiment was designed to test whether the agent would attack $P$. xylostella pupae on their host plant and if the presence of leek moth pupae on leek in the same environment would affect the frequency with which $P$. 
xylostella was attacked. Trials began with the introduction of three identically aged $D$. pulchellus females and were terminated after 24 hours. Once parasitoids were removed, the pupae were stored in marked Petri dishes and monitored to record pupal mortality and parasitoid emergence. All of the last three experiments had both positive and negative controls to measure parasitoid condition and host quality, respectively.

\subsubsection{Data Analyses}

The chi-square test for association was used to test the null hypothesis that $D$. pulchellus age ( 3 vs. 10 days) had no effect on the number of non-target hosts killed or on the number of non-targets yielding healthy parasitoid offspring from the no-choice tests. The same test was used to assess whether there were differences in host mortality and progeny production for each non-target species compared to the target host. For nontargets from which no $D$. pulchellus offspring emerged, chi-square tests were used to determine whether non-target mortality was greater in the presence of parasitoids than in the negative controls. Since positive controls, using both young and old parasitoids on leek moth, were run for each non-target species tested, there were 12 independent samples of the impact that these parasitoids had on the target pest. A paired t-test was used to determine whether parasitoid age affected the rate of host mortality and progeny production. Chi-square tests were also used for all analyses involving data from the Petri dish choice tests with leek moth and $P$. xylostella. Finally, the total mortality of target and non-target hosts from the four treatment groups in the cage choice tests were compared using ANOVA.

\subsection{Results}

\subsubsection{Simultaneous, No-choice, Black-box Test}

All of the 3- and 10-day-old parasitoids survived the full 24-hour no-choice exposure trial. Of the 12 non-target species offered to $D$. pulchellus females, only three (A. incertella, P. xylostella and $P$. porrectella) proved to be suitable for parasitoid development. When parasitoids encountered these non-target hosts, they were almost always arrested by contact with the cocoons, which they inspected intensely with their

antennae. One additional non-target, $Y$. dentella, was occasionally seen to elicit a 
response in $D$. pulchellus (see below). In contrast, there were no observations of a strong response to eight of the other non-targets. The three suitable non-target species had cocoons which most closely resembled those of the target host. As with leek moth, a $D$. pulchellus female had to thrust its abdomen into the host's cocoon through an opening at the end to achieve oviposition.

Parasitoid age did not have a significant effect on the likelihood of attack on the three suitable non-targets (Figure 5-1). Non-target mortality and parasitoid offspring emergence were similar in trials with young and old parasitoids $(P>0.05$ in all cases). Attacks appear to have been most frequent on $A$. incertella and least common on $P$. porrectella, and this relationship was consistent for the two parasitoid age groups. Further evidence of a preference for certain non-target species arises when the results from nontarget trials are compared to those of the corresponding positive controls. Diadromus pulchellus killed $A$. incertella at the same frequency as leek moth but $P$. porrectella significantly less often than leek moth (Figure 5-2). A significant difference between $P$. xylostella and leek moth mortality was detected for 10-day-old females and 3-day-old females demonstrated a similar, but non-significant, pattern. When progeny production is considered, again the only highly significant differences were between $P$. porrectella and leek moth (Figure 5-3). No differences were detected for either age treatment with $A$. incertella or P. xylostella.

There was no evidence of parasitoid-induced mortality or parasitoid development in the non-target $Y$. dentella. Nonetheless, parasitoids were occasionally seen to attack this species during the first few minutes of the trial. This pattern of intermittent attacks on $Y$. dentella provided an additional opportunity to look for age-related differences in motivation to oviposit by $D$. pulchellus. For the final 14 replicates of no-choice tests with Y. dentella, each parasitoid was observed from the moment it was released into the arena until it had either attacked the non-target or rejected it twice in a row (rejection = parasitoid not arrested by contact with host). This period normally lasted only a few minutes since the parasitoids were very active when introduced into the Petri dish. Older females attacked $Y$. dentella in five of the 14 trials whereas no young parasitoids demonstrated oviposition behaviour during the initial two host encounters. A Fisher exact test shows this difference to be significant $(P=0.020)$, however the sample size is too 
small to confidently conclude that the older females have a lower acceptance threshold. Furthermore, 3-day-old parasitoids were among the individuals seen attacking $Y$. dentella in the earlier no-choice trials.

Comparing the attack rates on leek moth by young and old parasitoids in the positive controls also suggests that the older females have a higher motivation to oviposit. Host mortality was significantly higher in the old treatment $(87.7 \pm 1.8 \%)$ than the young treatment $(80.2 \pm 1.4 \%)(t=-3.289 ; P=0.003)$. Since host quality was standardised among treatments, the higher frequency of host mortality with 10-day-old females is due to more frequent and/or more aggressive attacks by these parasitoids. An analysis of progeny production by young $(47.3 \pm 4.1 \%)$ and old $(43.2 \pm 3.2 \%)$ females failed to detect an age effect $(t=0.769 ; P=0.450)$.

\subsubsection{Choice Tests Between Acrolepiopsis assectella and Plutella xylostella} Direct Choice Test

When parasitoids were presented with a leek moth pupa next to a $P$. xylostella pupa, there was a very strong preference for the target species. Out of 30 trials, only two females - one 3- and one 10-day-old - chose $P$. xylostella by initiating oviposition behaviour on this host first. Due to the close proximity of the two hosts, the parasitoids inevitably antennated both hosts, even though the first cocoon encountered was random. Both hosts stimulated intense inspection by $D$. pulchellus involving antennating and biting the cocoon. However, in all cases where the agents were first arrested by $P$. xylostella, they showed a clear preference for the leek moth host once their antennae contacted its cocoon. In the two trials where D. pulchellus "chose" P. xylostella, the parasitoids actually abandoned the leek moth host and moved over to the non-target.

\section{Petri Dish Choice Test $(1: 1)$}

When $D$. pulchellus females were introduced into a small arena containing one leek moth and one $P$. xylostella pupa separated by $5 \mathrm{~cm}$, the non-target host was equally likely as leek moth to be attacked first by both young $\left(\chi^{2}=2.925 ; P=0.087\right)$ and old $\left(\chi^{2}=\right.$ $1.569 ; P=0.210$ ) females. Despite the equivalent probability of first attack, there were clear differences between the target and non-target with respect to host mortality (Figure 
5-4a) and progeny production (Figure 5-5a). Pupal mortality and parasitoid emergence were significantly greater in leek moth for both young and old parasitoid treatments $(P<$ 0.01 in all tests). No age-effect was detected for the number of $P$. xylostella pupae killed $\left(\chi^{2}=0.000 ; P=1.000\right)$ or successfully parasitised $\left(\chi^{2}=0.287 ; P=0.592\right)$.

Petri Dish Choice Test (1:4)

Increasing the non-target to target ratio in the choice test appeared to affect only the 10-day-old parasitoids. There was no change in the probability of first attacking the nontarget; $P$. xylostella was again as likely as leek moth to be the first host attacked by both young $\left(\chi^{2}=0.939 ; P=0.333\right)$ and old $\left(\chi^{2}=0.067 ; P=0.796\right)$ parasitoids. As in the previous experiment, leek moth hosts were significantly more likely to be killed (Figure $5-4 \mathrm{~b} ; \chi^{2}=7.500 ; P=0.006$ ) and to yield $D$. pulchellus offspring (Figure $5-5 \mathrm{~b} ; \chi^{2}=$ 10.335; $P=0.001)$ than $P$. xylostella when exposed to young parasitoids. In contrast, old females were equally likely to kill $\left(\chi^{2}=1.964 ; P=0.161\right)$ and produce progeny $\left(\chi^{2}=\right.$ $0.000 ; P=1.000$ ) in the non-target when it was more abundant than leek moth. Further analyses comparing $P$. xylostella mortality and parasitoid progeny production between the $1: 1$ and 1:4 host ratio experiments confirm that 10-day-old $D$. pulchellus females were less host specific under higher non-target densities (host mortality: $\chi^{2}=7.937 ; P=$ 0.005 ; progeny production: $\chi^{2}=4.286 ; P=0.038$ ). No such differences were found for the 3 -day-old parasitoid treatments (host mortality: $\chi^{2}=0.067 ; P=0.796$; progeny production: $\chi^{2}=0.069 ; P=0.793$ ). Due to the enhanced willingness of older parasitoids to attack $P$.xylostella pupae in the 1:4 experiment, these parasitoids killed non-targets significantly more often than young ones did $\left(\chi^{2}=9.320 ; P=0.002\right)$. There was, however, no difference in the number of trials with $D$. pulchellus emerging from $P$. xylostella $\left(\chi^{2}=1.669 ; P=0.196\right)$.

\section{Cage Choice Test}

Foraging $D$. pulchellus females readily attacked $P$. xylostella on canola, both in the absence and presence of the target host on leek. The probability of at least one $P$. xylostella being killed was consistently high in all treatments and did not differ from that of leek moth when the preferred host was present (Figure 5-6). In all trials, the parasitoids 
killed most of the available target and non-target hosts (Figure 5-7); the mortality rate was not affected by parasitoid age or the presence of leek moth hosts $(F=0.642 ; P=$ $0.591)$.

\subsection{Discussion}

This study helps to clarify the fundamental host range of Diadromus pulchellus Wesmael, a candidate agent for the classical biological control of leek moth in Canada. One objective was to determine whether $D$. pulchellus would accept and could develop in non-target species of varying taxonomic and ecological relatedness to leek moth. The second aim was to investigate how varying the physiological state of the agent could affect the outcome of host specificity tests. Parasitoid age did not have a substantial impact on host range expression. In no-choice tests, young and old females attacked the same four non-targets and their offspring emerged from the same three. Despite the failure of these response variables to detect any age effects, there was some evidence that 10-day-old parasitoids were more aggressive than their younger counterparts. For instance, older $D$. pulchellus females were more likely to attack non-target $Y$. dentella during the first one or two contacts with this host. Second, older females killed the leek moth hosts more often in the positive controls of the no-choice test. Finally, the 10-dayold parasitoids were more likely to kill a $P$. xylostella pupa in the 1:4 ratio choice tests. Although the results show only a weak age-effect, they do corroborate the concerns of several authors (e.g., Browne, 1993; Roitberg, 2000; Browne and Withers, 2002) who stressed that the condition of agents may affect the outcome of host specificity trials.

What are the implications of a minor physiological effect for host specificity testing? It would seem that if acceptance trials are not adequately replicated, there is a greater risk of obtaining false-negative results (i.e., results that incorrectly identify a nontarget as unsuitable (sensu Marohasy, 1998)) when the agent's motivation to oviposit is not maximised. Due to inaccessibility of certain non-targets or difficulty in rearing them, sample sizes are sometimes disturbingly small (e.g., Rogers and Potter, 2004; Goldson et al., 2005). When only a few individuals have been tested, the results, while not entirely meaningless, preclude drawing strong conclusions. This is especially the case when there 
is no evidence of attack since it is much more difficult to conclude that the non-target is not at risk (Hoffmeister et al., 2006). In these instances, the physiological state of the agent is likely to be an important factor.

These findings are relevant in light of recently proposed guidelines for host range testing of entomophagous agents. Withers and Browne (2004) composed a list of suggestions for controlling the physiological state of agents and conducting host range tests that would help to maximise host range expression. However, the "ideal state" of a parasitoid may not be universal among all species. For instance, Turlings et al. (1989) showed that prior experience with one host increased responsiveness of parasitoids to an unfamiliar host; while Kitt and Keller (1998) revealed that a previous host experience reduced the likelihood of accepting unfamiliar hosts. Furthermore, an increased readiness to oviposit in a familiar host does not necessarily directly translate to an increased readiness to attack a strange host. In the previous chapter, 3-day-old $D$. pulchellus females were shown to kill more leek moth hosts and produce more offspring than their 10-day-old siblings, suggesting that younger parasitoids had a higher motivation to oviposit. Based on this result, the younger females were expected to show higher attack frequencies during non-target trials. On the contrary, the older agents appeared to be slightly more aggressive in the present study. While this observation conforms to Withers and Browne's (2004) prediction that older agents should generally be more willing to parasitise non-targets, it illustrates that one may not be able to identify an agent's socalled "ideal state" based on observations of state-dependent responsiveness to the target host.

The results described above demonstrate that this parasitoid is stimulated to oviposit by contact with the cocoons of hosts that are closely related to the target. These acceptable non-targets include a species (A. incertella) in the same genus as leek moth, and two species ( $P$. xylostella and $P$. porrectella) in a very closely related family. Moreover, these three host species were suitable, albeit to varying degrees, for parasitoid development. The non-target $Y$. dentella, which belongs to a more distantly related family within Yponomeutoidea, only rarely stimulated oviposition behaviour. Despite occasional oviposition attempts on $Y$. dentella, no parasitoids emerged from these hosts. This outcome could have resulted from either rejection of the host following ovipositor 
insertion or failure of the parasitoid offspring to develop. The latter explanation is the most probable one given Thibout et al.'s (1988) observation that D. pulchellus appears to be less selective in the final stage of oviposition when its ovipositor is inside the host.

Although simultaneous, black-box, no-choice tests do not provide information on host preference per se, it is possible to rank non-targets based on their suitability for parasitoid development. This is done by examining the relative difference in host mortality or progeny production between each non-target and the simultaneous positive controls. A visual inspection of Figures 5-2 and 5-3 immediately reveals that $A$. incertella was equally suitable as a host to $D$. pulchellus whereas $P$. porrectella was far less suitable than leek moth. The rates of host mortality and parasitoid offspring emergence from P. xylostella were only marginally different from those of the target, suggesting that P. xylostella is also highly suitable for the agent's development. As host pupae were not dissected to check for evidence of oviposition, it is not possible to clarify whether differences in host mortality and parasitoid survival were due to variability in the intensity of parasitoid attack or in host immune response. Variation in host immunity is common within a species (Stoehr, 2007) and among related species (Brodeur and Vet, 1995); therefore the relatively low suitability of $P$. porrectella may have resulted from a higher rate of encapsulation of parasitoid eggs. Regardless of the underlying mechanism, the conspicuous difference in D. pulchellus emergence from the two Plutella species suggests that some members of Plutellidae would be entirely unsuitable for this parasitoid, especially if they do not feed on sulphur-rich crucifer hosts like $P$. xylostella and $P$. porrectella do.

While much more distantly related non-targets can support $D$. pulchellus development (Thibout, 1988), this is considered an improbable phenomenon since $D$. pulchellus will oviposit into these hosts only when the latter are placed inside the cocoon of an attractive host. This highlights the importance of the host's cocoon in the recognition process (Bekkaoui and Thibout, 1993; Gauthier et al., 2004) and suggests a significant degree of host specificity. Indeed, while naked leek moth pupae will be parasitised by $D$. pulchellus, they are less attractive than pupae kept inside the cocoon (Bekkaoui and Thibout, 1992). Despite being reared on attractive Brassica spp., three of the distantly related non-targets (Mamestra configurata, Spodoptera exigua and Pieris 
rapae) did not stimulate $D$. pulchellus. Their complete rejection may have been due to their lack of a silk cocoon; on the other hand, Bekkaoui and Thibout (1993) noted that extracts from the cocoon of a leek-reared generalist moth, Cacoecimorpha pronubana (Hübner) (Lepidoptera: Tortricidae), were only mildly attractive to $D$. pulchellus, in contrast to the strong probing response shown on extracts of leek moth cocoons.

Despite the nearly equivalent attack rates on $P$. xylostella and leek moth in nochoice tests, the choice test in which the two species were offered side-by-side demonstrated unequivocally that $D$. pulchellus has a strong preference for the target host. While this information may be useful to a point, the experimental design is unnatural since it would be very rare to find these two host species pupating right next to one another. The most likely scenario is that these pupae would occur metres apart on separate substrates. As demonstrated in the two Petri dish choice tests where the target and non-target pupae were separated by $5 \mathrm{~cm}$, adding even a small distance between the hosts can change the way these parasitoids respond to the lower ranked non-target. In these experiments, $D$. pulchellus females were equally likely to be arrested by and to attack a $P$. xylostella pupa before the target pupa. This is presumably due to the failure of the parasitoid to recognise the presence of the preferred host, despite it being only a few centimetres away. As Lecomte and Thibout (1983) showed, D. pulchellus females do not respond to volatile chemicals from the host and its cocoon; thus, unless the parasitoid previously or simultaneously contacts the leek moth host, the experiment cannot be considered a true choice test. Instead, it resembles a sequential no-choice test. What may be of interest in this case then is whether the parasitoid first contacts the target or nontarget host. In these so-called choice tests with equal (1:1) and unequal (1:4) host ratios and both young and old parasitoids, there was no apparent effect of the host species first contacted on the probability of a P. xylostella host being killed. The fact that $P$. xylostella was attacked in most of the trials may be an artifact of the long exposure period (24 hours). A test period of 3-5 hours might have revealed host preferences. Nonetheless, the aim here was to design tests that would maximise the likelihood of non-target acceptance (Withers and Browne, 2004).

Once it was observed that D. pulchellus would parasitise P. xylostella without habitat cues, the cage choice tests were conducted to assess how non-target acceptance 
was affected by the presence of the hosts' respective host plants. Canola (Brassica rapae), on which $P$. xylostella was reared and commonly feeds in the field, had no deterrent effect on $D$. pulchellus as these parasitoids were often observed investigating the sprigs in the BugDorm cages. Although a formal measurement was not taken, the parasitoids were equally likely to be spotted on the canola leaves as on the leek leaves when both were present. Thus, the presence of leek moth pupae on leek leaves did not reduce the tendency of $D$. pulchellus to parasitise $P$. xylostella. Again, these results may be an artefact of the experimental design (i.e., prolonged confinement) (Sands and Van Driesche, 2000), however, it demonstrates that this agent may occasionally search brassicaceous plants in the field. A field cage or open field test would be necessary to conclude whether $D$. pulchellus does naturally forage on plants outside the Allium genus.

The laboratory experiments in this study focused on the final stage of the foraging process where the agent encounters a potential host. Thus, host acceptance was determined strictly by the agent's response to the host's cocoon and/or body. In a natural environment, host selection by parasitoids generally involves a hierarchy of decisions and behavioural activities (Vinson, 1984); therefore, valuable information on foraging behaviour is lost when the earlier steps in host searching (i.e., habitat and host plant location) are excluded (Kitt and Keller, 1998). It is commonly suggested that host range expression will be greater when only the final stage of search is included since each phase of the entire foraging process acts as a filter. At each level of the search hierarchy, the range of potential hosts is reduced due to the exclusion of certain habitats. Keller (1999) explained that this filtering process does not necessarily resemble a series of increasingly fine sieves, since earlier steps in host search may be more restrictive than later ones. For instance, Thibout et al. (1988) proposed that D. pulchellus uses specific plant volatile cues for habitat and host location, as well as for host recognition, but then becomes rather unselective during the process of oviposition. Despite the general awareness of risking an inflated host range when studying only the final segment of the search process, most studies to date have chosen this approach as a starting point since these tests can be executed more quickly and with fewer resources. In the current study, the proposed agent was previously suspected to have a relatively restricted host range and these tests are effective in that they give very robust negative results that provide 
convincing evidence that a test species would not be attacked under field conditions. As suggested by van Lenteren et al. (2006), if simple host specificity tests are insufficient to conclude that non-targets are likely to be attacked in the field, then larger-scale and more natural tests can be conducted for those species which are less clear.

The results from this study suggest that the fundamental host range of $D$. pulchellus likely includes several species within the families Acrolepiidae and Plutellidae, but not likely outside these groups. However, there is a very low probability that this agent's ecological host range would include more than a few species within these two families. This proposition is supported by at least three lines of evidence. (1) A comprehensive literature search and discussions with European ichneumonid specialists failed to identify any field records of $D$. pulchellus from hosts other than leek moth in its native range, despite many years of study of this parasitoid by multiple research groups. If $D$. pulchellus were to attack $P$. xylostella as frequently in the field as it did in these laboratory tests, then this host association surely would have been reported among the immense number of European field studies on this major crop pest (e.g., Voukassovitch, 1927; Lugowska, 1981; Mustata, 1992). (2) The host lists of other Diadromus species, including one commonly used biological control agent, Diadromus collaris (Gravenhorst), consist of no more than three species (Yu et al., 2005). (3) Finally, D. pulchellus is strongly attracted to plants, particularly host-damaged plants, which release sulphur-containing volatile compounds (Lecomte and Thibout, 1983) and are also stimulated to oviposit by specific sulphur-rich proteins found in leek moth cocoon silk (Gauthier et al., 2004).

Based on what is known of the search behaviour of D. pulchellus, there should be minimal risk to non-targets occurring on host plants without a distinct sulphur volatile signature. This parasitoid likely forages primarily, if not exclusively, in habitat with Allium and brassicaceous plants. While non-target species in the family Plutellidae are less related to leek moth than the acrolepiids, they tend to colonise brassicaceous plants. These non-targets would therefore be among the most likely to be encountered by this agent in the field. The two plutellids tested here are common in southeastern Ontario and southwestern Quebec where leek moth is currently found, however neither species is native to North America. In fact, no native Plutella species are known to exist in the 
agricultural zones of eastern North America. The only recorded species from the entire Plutellidae family in this large region is Rhigognostis interrupta Walsingham (Lepidoptera: Plutellidae), but nothing is known of its life history (J.-F. Landry, personal communication). Thus, in the region of eastern North America where leek moth is expected to spread in the foreseeable future, the ecological host range of $D$. pulchellus should be restricted by the absence of species that it might be capable of locating and parasitising.

Despite being more closely related to leek moth, the non-target members of Acrolepiidae should fall outside the ecological host range of $D$. pulchellus since their known host plants do not release volatile sulphur compounds. Acrolepiopsis heppneri and $A$. incertella have distributions covering much of the eastern United States and possibly the southern region of Ontario, Canada. Despite inevitably one day having an overlapping distribution with the invasive leek moth, these non-targets exist in a very different habitat than the pest. Leek moth is a vegetable crop pest, whereas $A$. heppneri and $A$. incertella feed on Smilax spp., which grow in deciduous forests, often along stream banks.

In summary, parasitoid physiological state can influence non-target host acceptance, however the importance of this factor may depend largely on the test design and number of replicates. For instance, Withers and Browne (2004) were correct in predicting that non-targets were more likely to be attacked in choice tests when offered in higher numbers than the preferred host; however, in the present study this pattern only emerged when using the older agents. When conducting choice tests to assess host preference, it is important to understand how an agent perceives its environment and locates its hosts. If the agent does not use volatile or visual cues for short-range detection of its host, it is possible that it will encounter and accept/reject a host without knowledge of the presence of alternative hosts. Such an experimental design can only be considered a true choice test if it is run long enough to allow the agent to properiy detect and assess all the available hosts. In this case, the order in which different host species are encountered may be an important predictor of non-target acceptance.

There has been some discussion of the importance of ecological similarity versus phylogenetic affinity between target and non-target hosts when considering which species are most at risk of non-target effects (Barratt et al., 1999; Messing, 2001; Kuhlmann et 
al., 2006). In the leek moth - D. pulchellus system it appears that phylogenetic affinity is a key indicator of non-target risk since all three of the suitable hosts were close relatives of leek moth even though only two feed on sulphur-rich plants. In addition, $Y$. dentella, which is likely the next most taxonomically related species (Dugdale et al., 1998), elicited oviposition behaviour in a few instances despite that its host plant does not produce the sulphur compounds that attract $D$. pulchellus. But given the selectivity of these parasitoids to plant odours, non-target host plant and pupation site are probably critical factors in predicting likelihood of encounter and attack in the field. The estimated fundamental host range of $D$. pulchellus is very likely broader than its ecological host range. The final step in verifying that these parasitoids are specific to one or a small number of hosts would be to clarify its habitat preference and selection behaviour. This could be done using wind tunnel assays as described by Kitt and Keller (1998) or open field trials in the agent's area of origin. 
Table 5-1. List of non-targets and their respecitve host plants for Diadromus pulchellus host specificity testing.

\begin{tabular}{|c|c|c|}
\hline $\begin{array}{l}\text { Insect Species } \\
\text { (Family) } \\
\end{array}$ & $\begin{array}{l}\text { Host Plant } \\
\text { (Family) }\end{array}$ & Motivation for Selection \\
\hline \multicolumn{3}{|l|}{ Target } \\
\hline $\begin{array}{c}\text { Acrolepiopsis assectella (Zeller) } \\
\text { (Acrolepiidae) }\end{array}$ & $\begin{array}{l}\text { Allium porrum (Linnaeus) } \\
\text { (Alliaceae) }\end{array}$ & -- \\
\hline \multicolumn{3}{|l|}{ Non-targets } \\
\hline $\begin{array}{l}\text { Plutella xylostella (Linnaeus) } \\
\text { (Plutellidae) }\end{array}$ & $\begin{array}{l}\text { Brassica napus (Linnaeus) } \\
\quad \text { (Brassicaceae) }\end{array}$ & $\begin{array}{l}\text { phylogenetic affinity } \\
\text { ecological similarity } \\
\text { morphological similarity } \\
\text { host of a Diadromus sp. }\end{array}$ \\
\hline $\begin{array}{l}\text { Plutella porrectella (Linnaeus) } \\
\text { (Plutellidae) }\end{array}$ & $\begin{array}{l}\text { Hesperis matronalis (Linnaeus) } \\
\text { (Brassicaceae) }\end{array}$ & $\begin{array}{l}\text { phylogenetic affinity } \\
\text { ecological similarity } \\
\text { morphological similarity }\end{array}$ \\
\hline $\begin{array}{l}\text { Acrolepiopsis incertella (Chambers) } \\
\text { (Acrolepiidae) }\end{array}$ & $\begin{array}{l}\text { Smilax tamnoides (Linnaeus) } \\
\text { (Smilacaceae) }\end{array}$ & $\begin{array}{l}\text { phylogenetic affinity } \\
\text { safeguard species } \\
\text { morphological similarity }\end{array}$ \\
\hline $\begin{array}{c}\text { Ypsolopha dentella (Fabricius) } \\
\text { (Ypsolophidae) }\end{array}$ & $\begin{array}{l}\text { Lonicera tatarica (Linnaeus) } \\
\qquad \text { (Caprifoliaceae) }\end{array}$ & $\begin{array}{l}\text { phylogenetic affinity } \\
\text { morphological similarity }\end{array}$ \\
\hline $\begin{array}{c}\text { Yponomeuta padella (Linnaeus) } \\
\text { (Yponomeutidae) }\end{array}$ & $\begin{array}{l}\text { Crataegus monogyna (Jacquin) } \\
\text { (Rosaceae) }\end{array}$ & phylogenetic affinity \\
\hline $\begin{array}{l}\text { Yponomeuta cagnagella (Huebner) } \\
\text { (Yponomeutidae) }\end{array}$ & $\begin{array}{l}\text { Euonymus europaeus (Linnaeus) } \\
\text { (Celastraceae) }\end{array}$ & phylogenetic affinity \\
\hline $\begin{array}{l}\text { Athrips mouffetella (Linnaeus) } \\
\text { (Gelechiidae) }\end{array}$ & $\begin{array}{l}\text { Lonicera tatarica (Linnaeus) } \\
\qquad \text { (Caprifoliaceae) }\end{array}$ & morphological similarity \\
\hline $\begin{array}{l}\text { Lobesia botrana (Denis \& Schiff.) } \\
\text { (Tortricidae) }\end{array}$ & $\begin{array}{l}\text { Vitis vinifera (Linnaeus) } \\
\text { (Vitaceae) }\end{array}$ & host of a Diadromus sp. \\
\hline $\begin{array}{l}\text { Plodia interpunctella (Huebner) } \\
\text { (Pyralidae) }\end{array}$ & stored grain mix * & morphological similarity \\
\hline $\begin{array}{c}\text { Spodoptera exigua (Huebner) } \\
\text { (Noctuidae) }\end{array}$ & $\begin{array}{l}\text { Brassica oleracea (Linnaeus) } \\
\text { (Brassicaceae) }\end{array}$ & ecological similarity \\
\hline $\begin{array}{l}\text { Mamestra configurata Walker } \\
\text { (Noctuidae) }\end{array}$ & $\begin{array}{c}\text { Brassica napus (Linnaeus) } \\
\text { (Brassicaceae) }\end{array}$ & ecological similarity \\
\hline $\begin{array}{c}\text { Pieris rapae (Linnaeus) } \\
\text { (Pieridae) }\end{array}$ & $\begin{array}{c}\text { Brassica oleracea (Linnaeus) } \\
\text { (Brassicaceae) }\end{array}$ & ecological similarity \\
\hline
\end{tabular}

\footnotetext{
* The stored grain mix consists of flour, almonds and rice.
} 

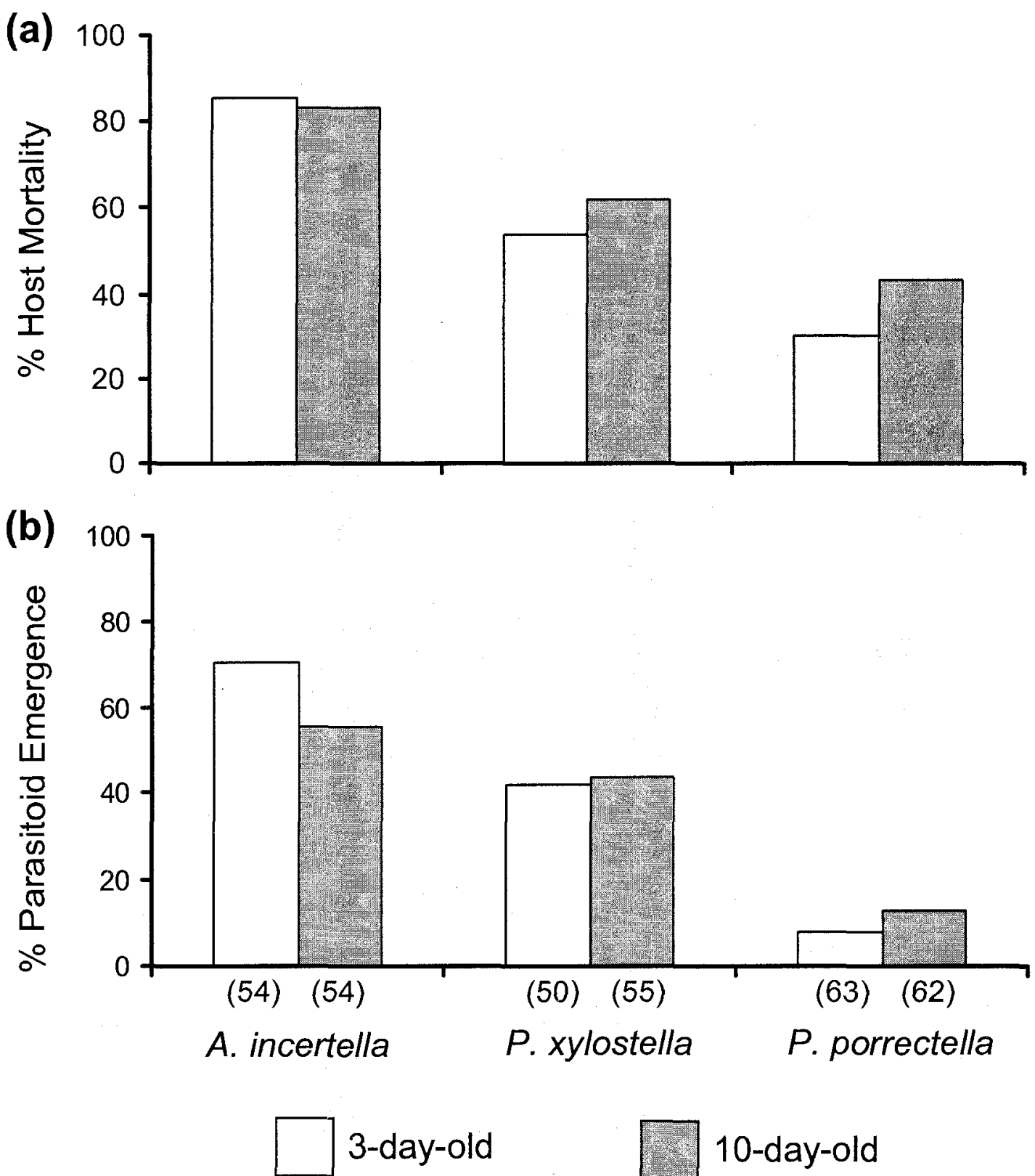

10-day-old

Figure 5-1. Effect of Diadromus pulchellus Wesmael female age on (a) the percentage of non-target hosts killed (i.e., failing to yield a non-target adult) and (b) the percentage of hosts yielding parasitoid adults in no-choice trials. The number of tested females for each treatment is given in parentheses at the base of each column. Parasitoid age did not have a significant effect on host mortality or offspring emergence. 
(a)

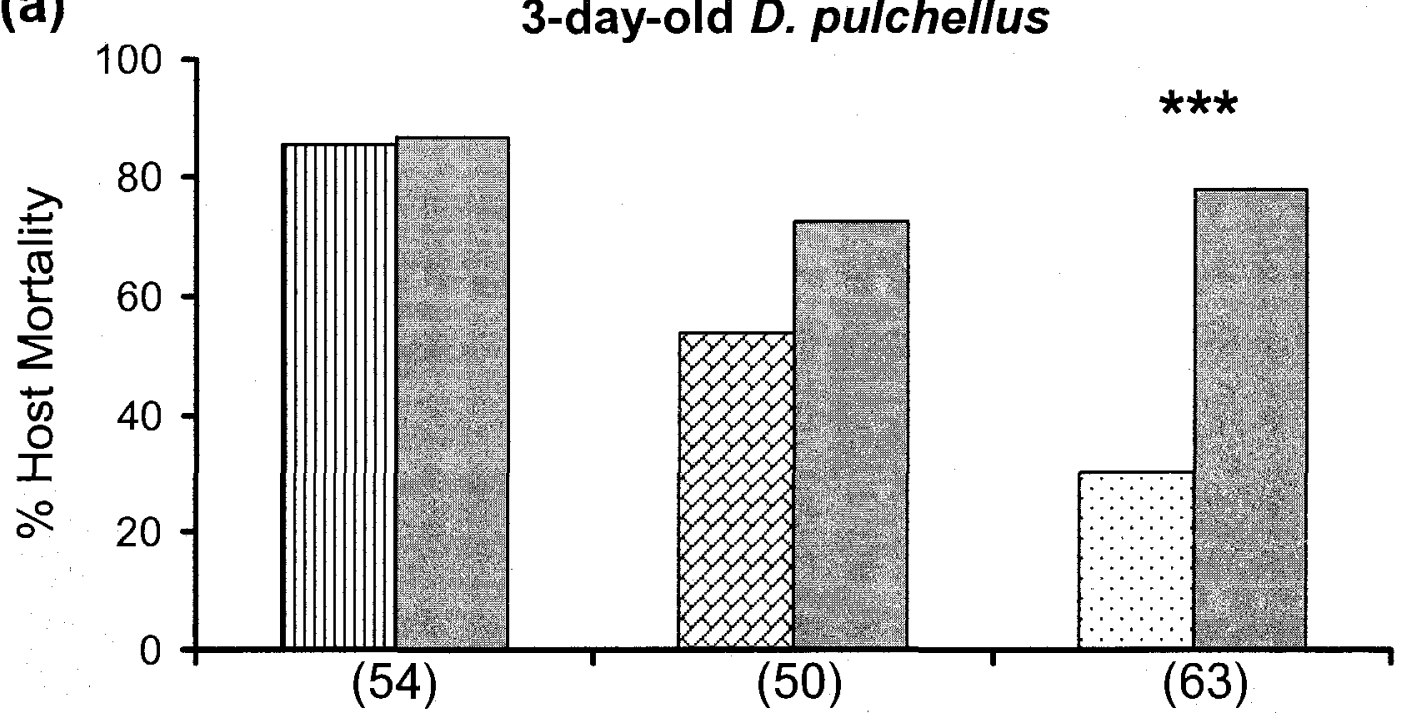

(b)
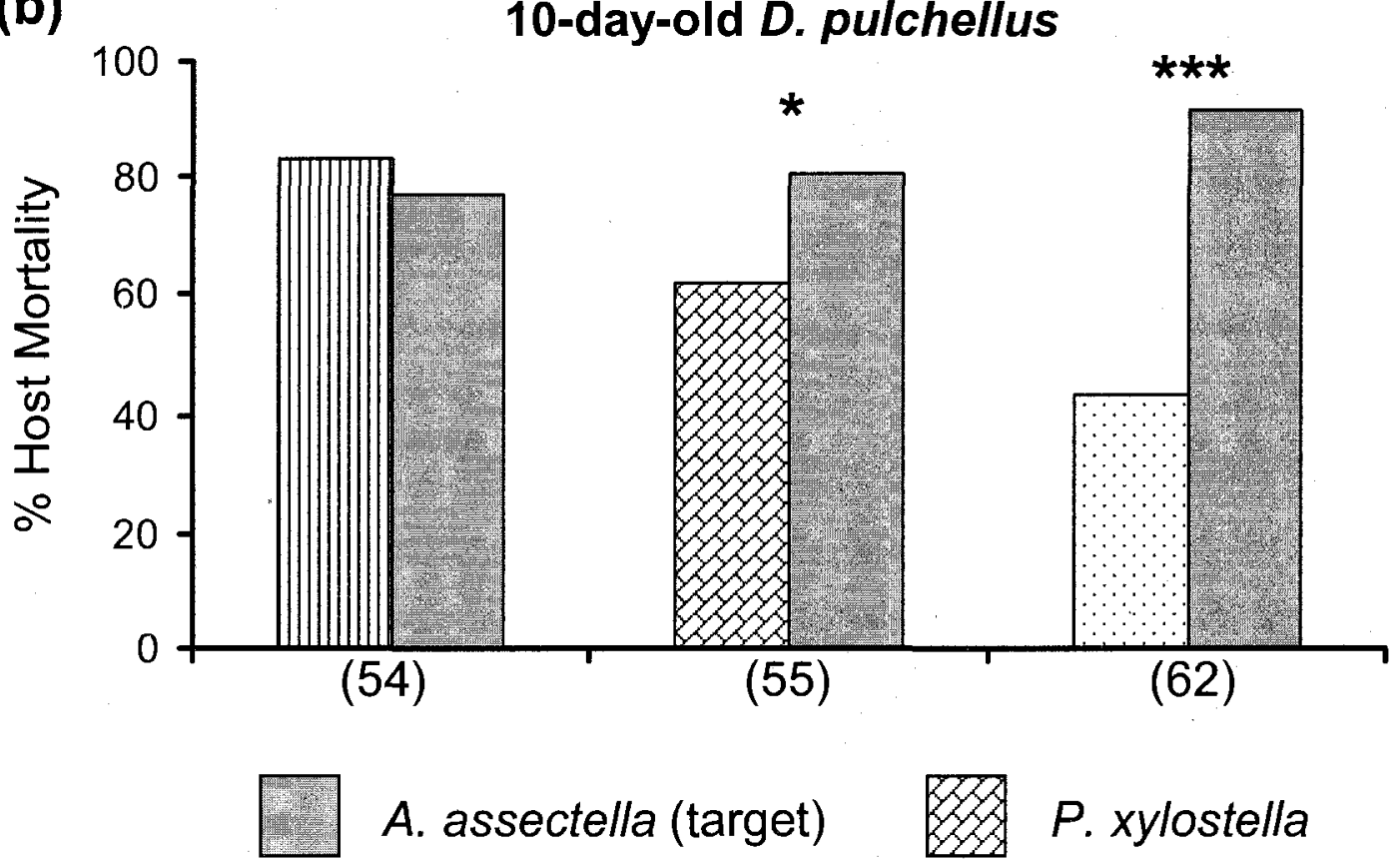

A. assectella (target)

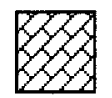

P. xylostella

A. incertella

P. porrectella

Figure 5-2. Percentage of no-choice trials in which non-target and target hosts were killed (i.e., failing to yield a moth adult) by (a) 3-day-old and (b) 10-day-old Diadromus pulchellus Wesmael females. Non-target species shown are Acrolepiopsis incertella (Chambers), Plutella xylostella (Linnaeus) and Plutella porrectella (Linnaeus), while the target is Acrolepiopsis assectella (Zeller). The number of trials for each treatment is shown in parentheses beneath the columns. ${ }^{*} \mathrm{P}<0.05 ;{ }^{* * *} \mathrm{P}<0.001$ 


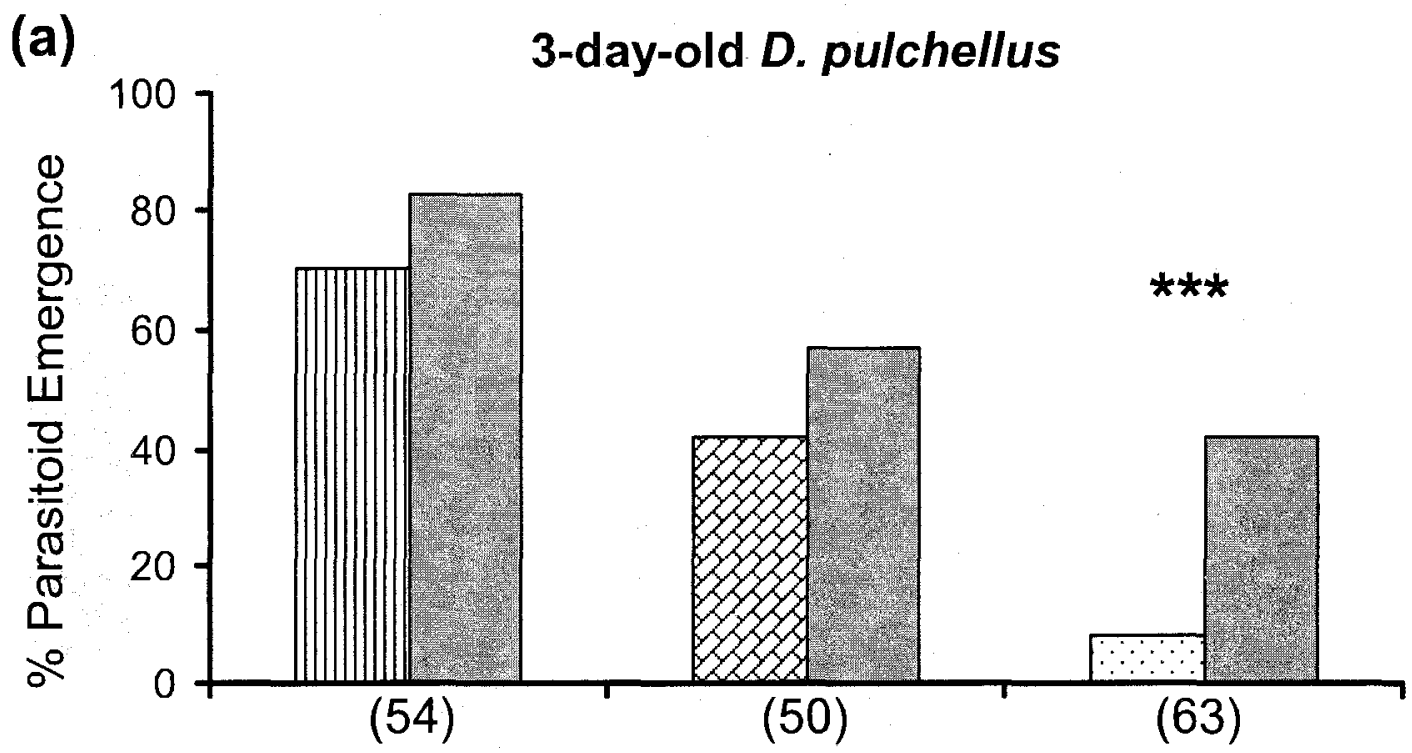

(b)
0
0
0
0
0
0
0
0
0
0
0
0
0

(b)

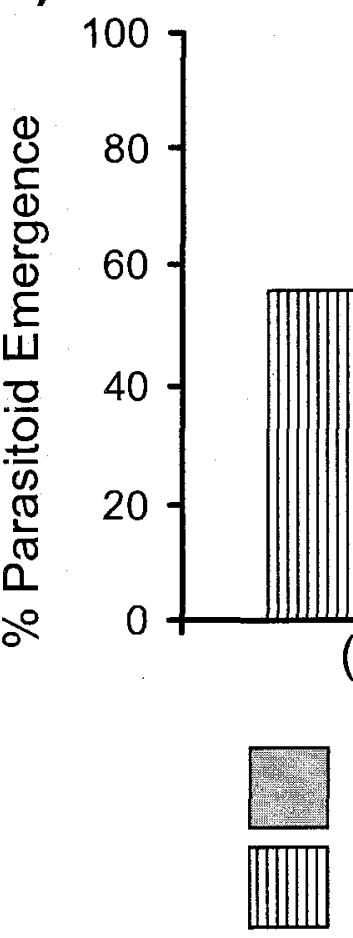

10-day-old D. pulchellus

(54)

(55)

(62)
A. assectella (target)

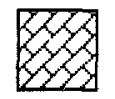
P. xylostella
A. incertella
P. porrectella

Figure 5-3. Percentage of no-choice trials in which non-target and target hosts yielded parasitoid offspring following exposure to (a) 3-day-old and (b) 10-day-old Diadromus pulchellus Wesmael females. Non-target species shown are Acrolepiopsis incertella (Chambers), Plutella xylostella (Linnaeus) and Plutella porrectella (Linnaeus), while the target is Acrolepiopsis assectella (Zeller). The number of trials for each non-target is shown in parentheses beneath the columns. $* * * \mathrm{P}<0.001$ 


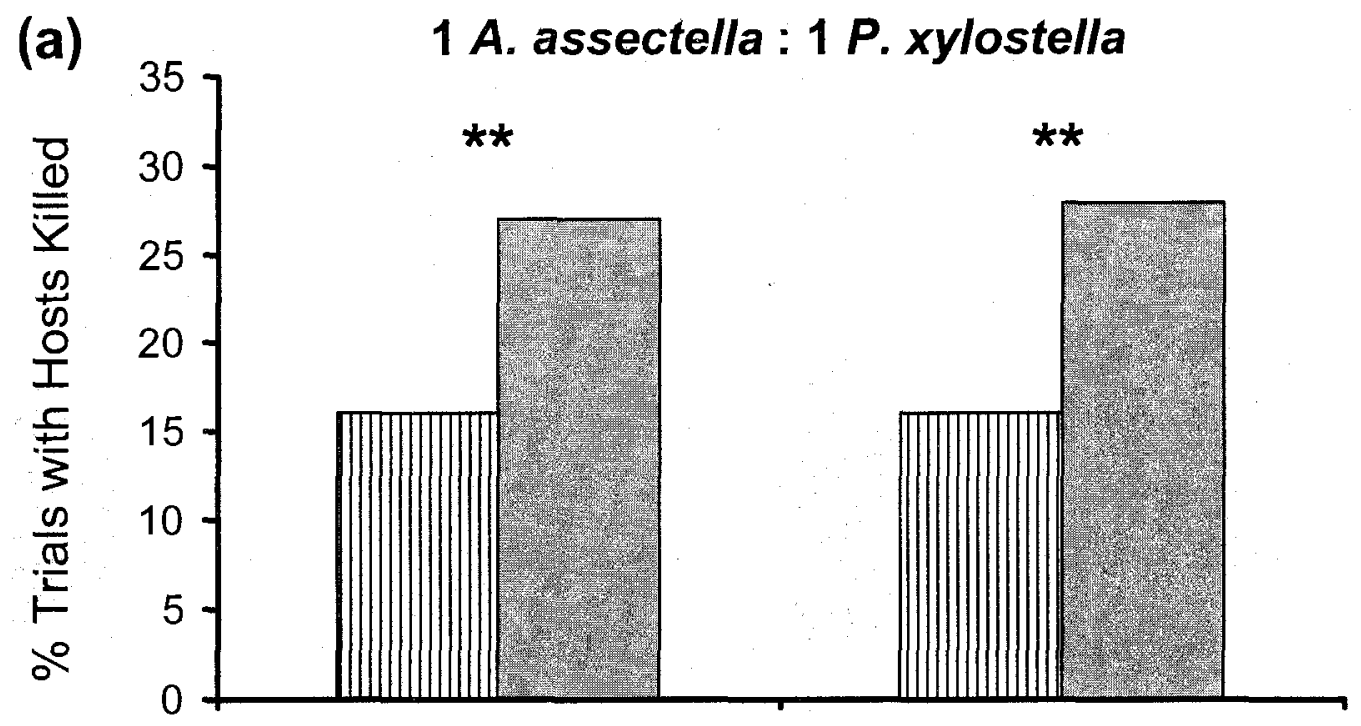

(b)

1 A. assectella : 4 P. xylostella

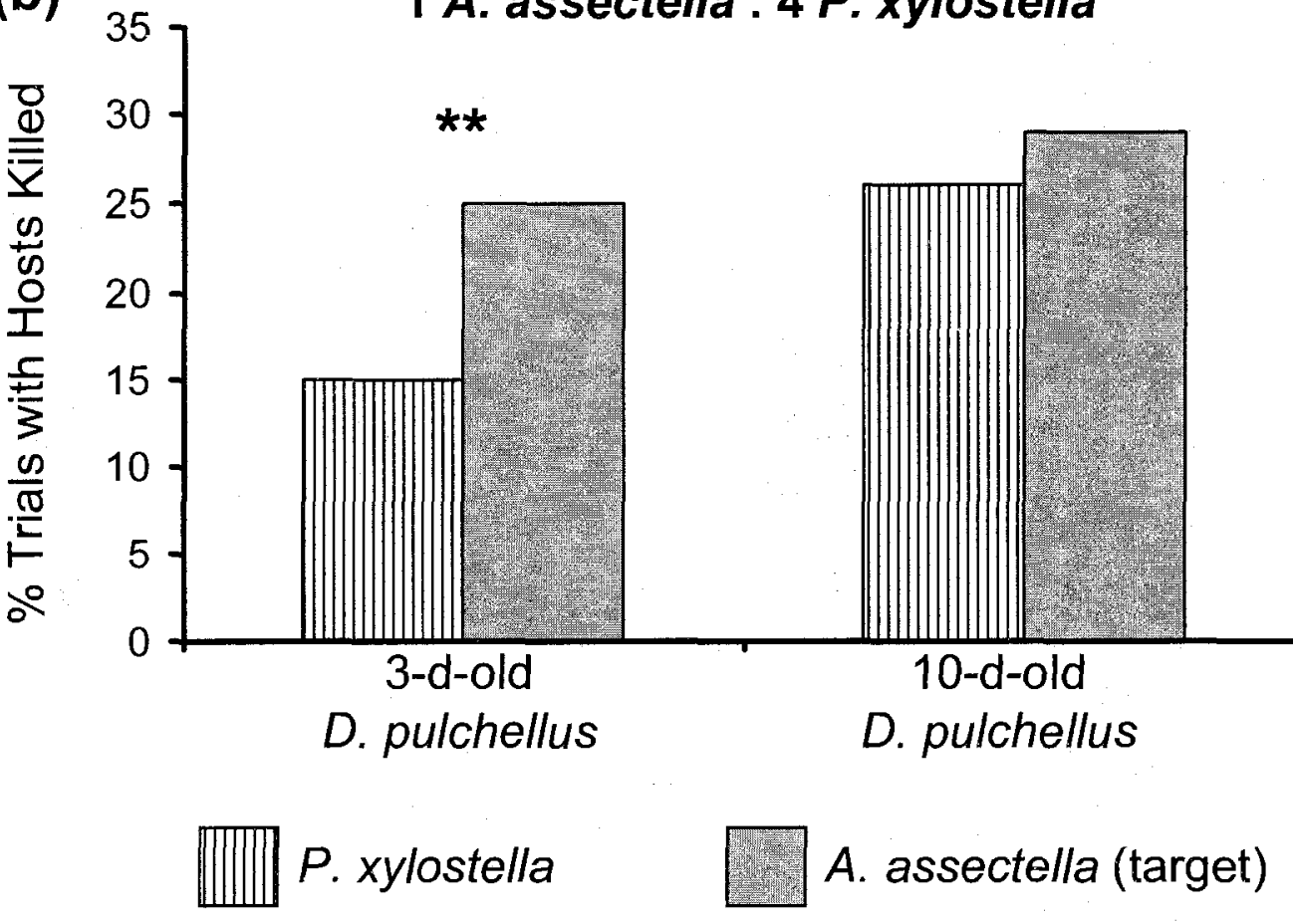

Figure 5-4. Percentage of Petri dish choice trials in which at least one non-target or target host was killed when a 3- or 10-day-old Diadromus pulchellus Wesmael female was offered (a) one Acrolepiopsis assectella (Zeller) pupa with one Plutella xylostella (Linnaeus) pupa or (b) one A. assectella pupa with four $P$. xylostella pupae. $* * P<0.01$. 

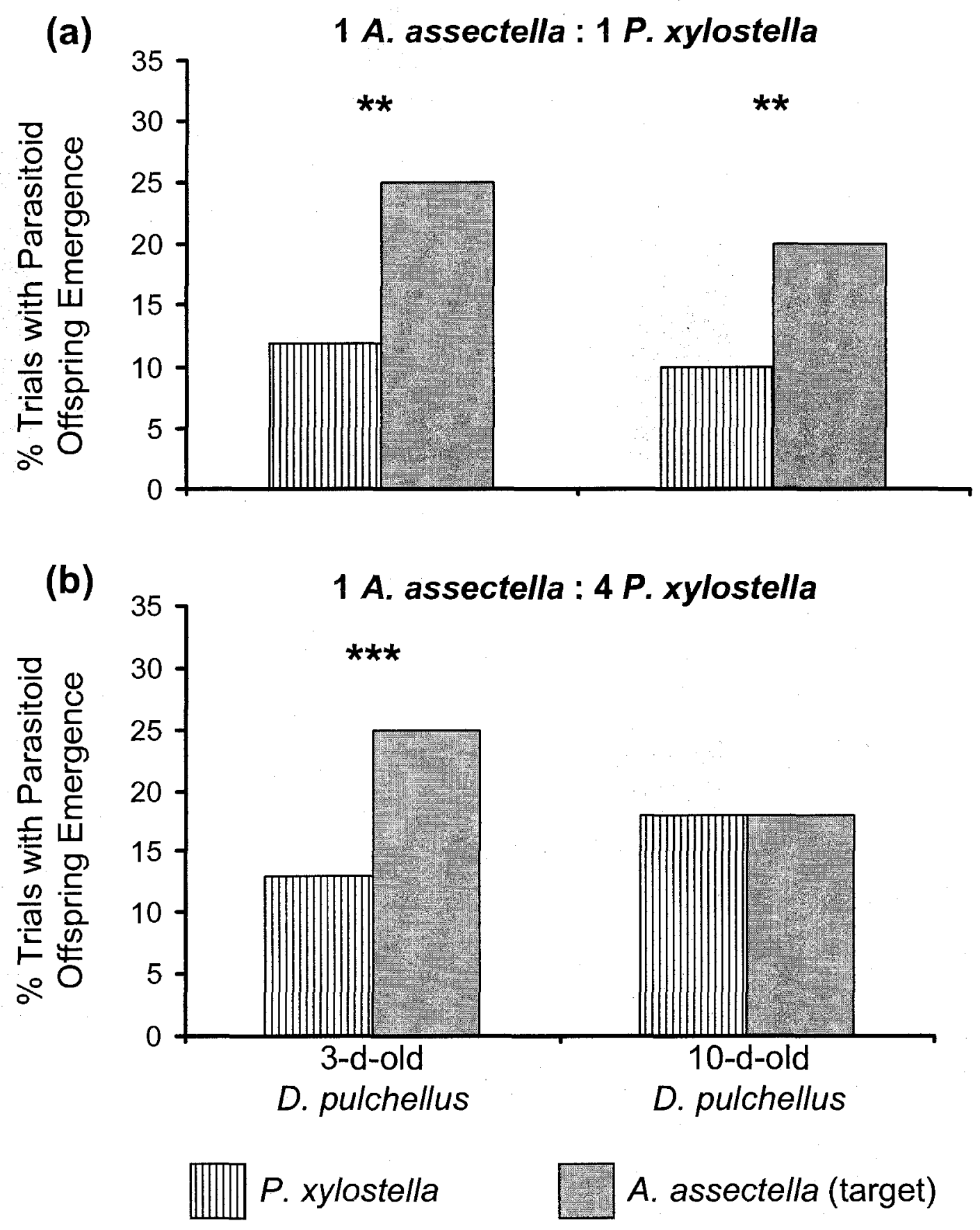

Figure 5-5. Percentage of Petri dish choice trials in which at least one non-target or target host yielded parasitoid offspring when a 3- or 10-day-old Diadromus pulchellus Wesmael female was offered (a) one Acrolepiopsis assectella (Zeller) pupa with one Plutella xylostella (Linnaeus) pupa or (b) one $A$. assectella pupa with four $P$. xylostella pupae. ** $P<0.01 ; * * * P<0.001$. 

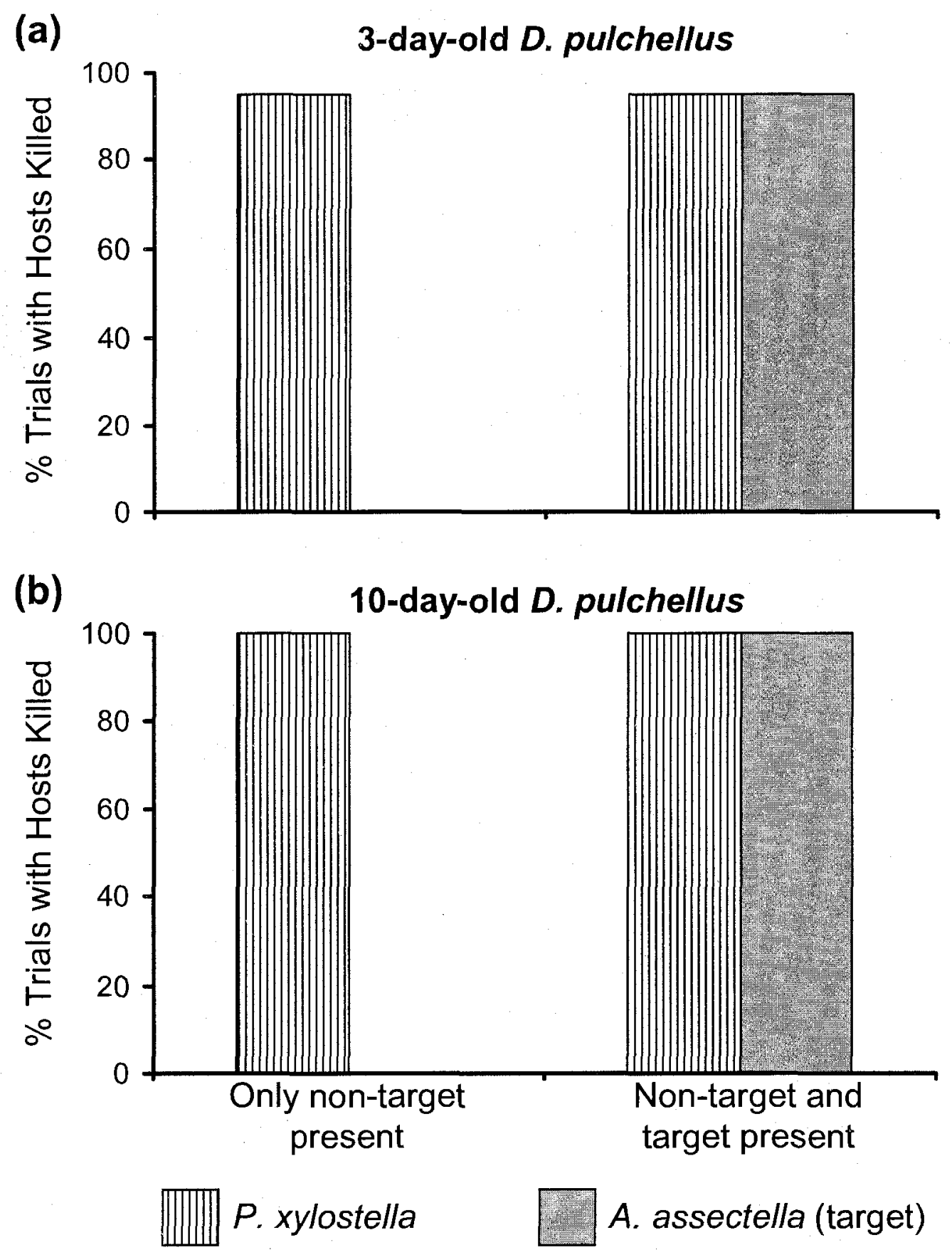

Figure 5-6. Percentage of cage trials in which at least one Plutella xylostella (Linnaeus) or Acrolepiopsis assectella (Zeller) were killed by (a) 3-day-old and (b) 10-day-old Diadromus pulchellus Wesmael females. When only non-target hosts were present, there were four $P$. xylostella pupae. In trials with both non-target and target hosts, there were two $P$. xylostella and two $A$. assectella pupae. 

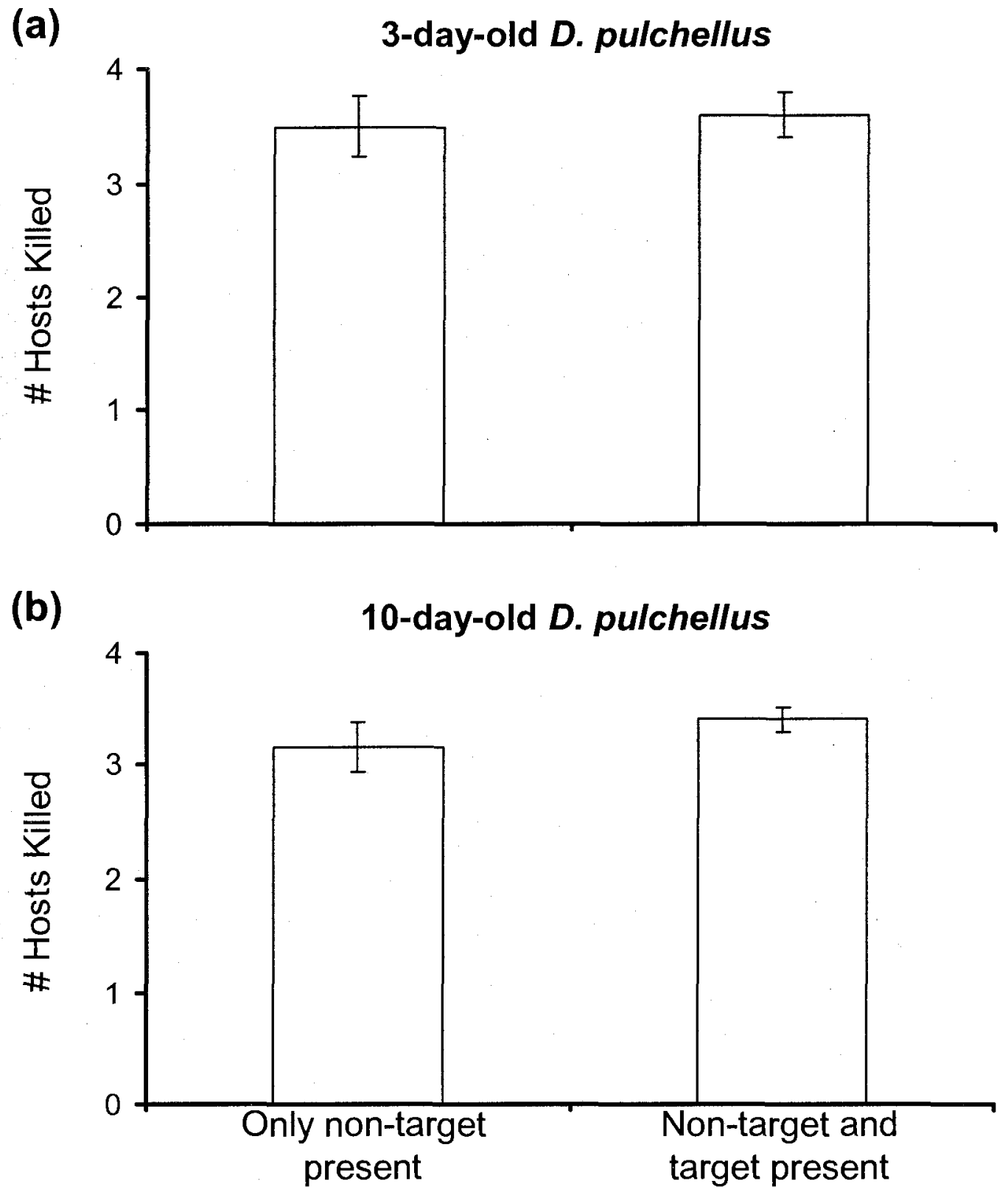

Figure 5-7. Mean number of hosts ( \pm S.E.) killed by Diadromus pulchellus Wesmael females in cage trials. When only non-target hosts were present, there were four Plutella xylostella (Linnaeus) pupae. In trials with both non-target and target hosts, there were two P. xylostella and two Acrolepiopsis assectella (Zeller) pupae. In trials with two host species, counts of killed hosts include both species. Neither parasitoid age nor the presence of the target host had a significant effect on the number of hosts killed in a trial $(P>0.05)$. 


\section{General Discussion}

Since its relatively recent and accidental introduction into Canada, leek moth, Acrolepiopsis assectella (Zeller) (Lepidoptera: Acrolepiidae), has posed an increasing risk to commercial and hobbyist Allium production in the country. Its continued spread throughout southeastern Ontario and southwestern Quebec means that more growers are affected each year. The wide distribution of leek moth in Europe, North Africa and Asia indicates that it has the potential to eventually colonise most of the cultivated regions of North America. Moreover, although this moth is traditionally associated with cultivated Allium spp., it may also colonise wild species of this genus in North America (Allison et $a l ., 2007)$. If left unchecked, leek moth populations can devastate food and seed crops (Plaskota and Dabrowski, 1986; Nyrop et al., 1989). It is clear that complete eradication of leek moth from Canada is not feasible; therefore, management approaches are needed to restrict the impact and rate of spread of this exotic pest.

Commonly grown Allium crops, such as leek, garlic and onion, tend to have a low diversity of pests. The onion thrips, Thrips tabaci Lindeman (Thysanoptera: Thripidae), and the onion fly, Delia antiqua (Meigen) (Diptera: Anthomyidae), are traditionally the two main insect pests of Allium in North America. The addition of a new and potentially severe pest species will inevitably complicate the production of these crops. Since leek moth larvae mine the upper central leaves of leeks and garlic, they occupy a separate niche from $T$. tabaci, which typically feeds between leaf blades, and $D$. antique, which bores into the lower stem and bulb. Thus, existing control measures for the original two pests may not be adequate for controlling leek moth. Starting in 2004, laboratory and field trials demonstrated that spinosad-based insecticides were relatively effective in reducing leek moth numbers (Mason et al., 2006). In addition, these authors found that placing row covers over crops during moth flight periods was the most effective management option as it prevented females from laying their eggs on the crop. Unfortunately, there is some concern over the practicality of this latter control method due to the work involved in covering and uncovering entire vegetable fields.

Biological control is one component of integrated pest management that can effectively reduce crop damage and requires little input from the growers themselves. 
This is particularly true for classical biological control, where an exotic agent is released for permanent establishment, often by government officials and therefore at no expense to growers. Despite the thousands of entomophagous species that have been released for classical biological control over the last century (Greathead, 1995), the process of selecting and releasing a suitable agent often still requires plenty of research to ensure that the best natural enemies are used. The general approach to introducing exotic agents has transformed from one in which natural enemies were selected almost exclusively for their killing power to a system where agents are thoroughly screened both to increase the likelihood of effective pest control and to minimise the risk to non-target species. The extent to which pre-release research must be conducted depends mainly on what was previously recorded in the literature and how reliable it is thought to be. Unreliability of existing information is a frequent problem in assessing an agent's host range due to misidentifications or erroneous host associations (Sands and Van Driesche, 2004).

This thesis describes research that examined the potential for classical biological control of leek moth in Canada. The first step was to examine the pest's survivorship in its native range and to survey for potential agents. Chapter 2 describes a life table study that measured age-specific mortality of the pest in the final generation at three sites and over three years. This experiment showed that natural enemies made a significant contribution to pupal mortality, suggesting that the pupal stage is particularly vulnerable to attack. This is not surprising since leek moth larvae spin their cocoons and pupate on the external surface of the plant whereas the larvae feed inside the leaves of the host plant. While leek moth eggs are also laid on the outside of the plant, they were never parasitised and predators did not appear to play a significant role in egg survival under field conditions. The tiny size and lower apparency of leek moth eggs may explain why they are not as vulnerable to predators as the pupae are.

Neonate larval mortality was one key factor affecting changes in population density. When leek moth larvae hatch on the surface of the host plant, they must penetrate the leaf surface to establish a protected feeding mine. The survival of newly hatched larvae was highly variable and appeared to be independent of natural enemies. It is possible that these minute larvae are easily dislodged from the plant by wind or rain. Due to the apparent frailty of neonate larvae, the period of egg hatch and establishment 
on the plant is also thought to be the most effective moment to spray an insecticide since the neonates are less tolerant than older larvae and they must ingest external plant tissue to burrow into the leaf.

Despite the nearly complete absence of parasitoids during the life table study, several hymenopteran species were reared from field-collected leek moth hosts. Leek moth parasitoids were obtained almost exclusively from field sites where pest densities were relatively high (i.e., leeks along crop edges were frequently infested). Among the parasitoid species reared out of leek moth larvae and pupae, Diadromus pulchellus Wesmael (Hymenoptera: Ichneumonidae) was the only species that warranted consideration for introduction into Canada since the remaining five species are known to have low host specificity. Diadromus pulchellus has been well-studied over the last three decades, but has never been used in a biological control context. Previous research on this agent has covered aspects such as mating and oviposition behaviour (Noyes, 1974; Kalmes and Rojas-Rousse, 1988a), host location and recognition cues utilised by females (Auger et al., 1989; Bekkaoui and Thibout, 1993; Thibout et al., 1993; Thibout, 2005), characterisation of an ascovirus associated with the parasitoid (Bigot et al., 1997), competitive interactions with congeneric parasitoids (Kalmes et al., 1983; Kalmes and Rojas-Rousse, 1988b) and, to a small degree, host specificity (Thibout, 1988; Bekkaoui and Thibout, 1993). Nevertheless, additional studies were needed to determine whether this natural enemy is suitable for introduction into North America.

The first step was to assess the cold tolerance and overwintering habit of $D$. pulchellus to estimate the probability of its survival through a Canadian winter. The overwintering strategy of $D$. pulchellus was not known prior to this work, but it was suspected to pass the winter in the adult stage. This was based on previous records of adult overwintering in congeneric species and on the observation that parasitoids emerge from their host pupae in the final leek moth generation at the end of the growing season. Two of the experiments described in Chapter 3 demonstrated that immature parasitoids did not tolerate long periods of sustained cool $\left(3\right.$ and $\left.8^{\circ} \mathrm{C}\right)$ temperatures or extended periods of fluctuating temperatures, which sometimes dropped below freezing. Parasitoid adults, on the other hand, were able to survive lengthy episodes of both constant and fluctuating temperatures, above and below $0^{\circ} \mathrm{C}$. While there would likely be substantial 
overwintering mortality of parasitoids, these results indicate that survival through a Canadian winter is probable, especially under a layer of insulating snow.

Perhaps the most important task in evaluating $D$. pulchellus for classical biological control was to measure its host specificity. While the concept of assessing a parasitoid's host specificity may, at first, seem straight forward, the process is complicated by a lack of a "best test". Experimental test design is an important factor that can influence the outcome of host range testing (Withers and Browne, 2004), but there is no universally preferred test. For instance, the optimal test design may vary according to the species and search behaviour of the agent, the host stage attacked, the behaviour of the host or even the work schedule of the researcher. To date, a wide variety of test designs have been employed for dozens of different candidate biological control agents. Unfortunately, too little is known about how accurately the different host range tests of entomophagous agents predict the likelihood of non-target impacts following introduction. This is due to the lack of post-release studies on non-target effects in previous programmes. Thus, it is currently impossible to prescribe a single, standardised host range testing protocol for all biological control agents of arthropod pests.

A set of guidelines for the general design of host range tests was given by van Lenteren et al. (2006), who recommended starting with simplified test environments and only increasing the complexity if false-positive results are suspected. They also stressed the importance of flexibility in deciding which tests to use. Withers and Browne (2004) provided more specific suggestions with respect to preparation of the agents prior to testing. Their recommendations were aimed at ensuring that parasitoids would have a high motivation to oviposit during specificity trials, since it is known that foraging decisions can be affected by parasitoid state and environmental factors (Briggs et al., 1999; Roitberg, 2000). A parasitoid's condition and the information it receives about its environment could conceivably influence the probability of it accepting a low quality, or unfamiliar, host. While this is a theoretical possibility, there is very little hard evidence showing that changes in a parasitoid's physiological and informational state can modify the non-target species that it is willing to parasitise. The final research chapters of this thesis investigate how the motivation of $D$. pulchellus to oviposit is affected by 
endogenous and exogenous factors and whether one such factor (age) can affect host range expression in non-target tests.

Motivation to oviposit, measured as the number of leek moth hosts killed (hosts that yielded a parasitoid or died prematurely) and parasitoid progeny produced over a 24-hour period, was affected by four of the seven factors tested in Chapter 4. Parasitoid age and nutritional status had strong effects on oviposition activity, while previous host experience and parasitoid density both had weaker and inconsistent effects. Parasitoid mating status, arena size and the presence/absence of the host plant did not have a detectable impact on the number of hosts killed or successfully parasitised. The most surprising result was the direction of the effect of age and nutritional status on apparent motivation to oviposit. Younger D. pulchellus females killed more hosts and produced more offspring than old females and sugar-fed individuals similarly showed signs of a greater reproductive drive than their starved siblings. This contradicts the predictions of Withers and Browne (2004) and suggests that physiological and environmental variables affect foraging behaviour of different species in different ways. An important consideration is that different behavioural parameters may not be affected in the same way, or to the same extent, by a single factor. Therefore, the behavioural attribute used for determining an agent's ideal state prior to host range testing (e.g., odour response, attack rate, superparasitism) should be relevant to the planned host range test design.

Although $D$. pulchellus age had a strong effect on motivation to oviposit in leek moth hosts, it had only a very marginal effect on the outcome of host specificity tests. The results in Chapter 5 show that, in no-choice tests, 3- and 10-day-old parasitoids attacked the same four non-target species. Among these, the three non-targets most closely related to leek moth were suitable for parasitoid development. An age-effect was observed in the choice tests, however detection of this effect was dependent upon the test design. Older parasitoids attacked non-target hosts more often in trials where the nontargets outnumbered the target host, whereas changing the host ratio had no effect on the likelihood of non-target acceptance by young females. The importance of parasitoid age for host specificity tests therefore depends on the goals of the research. If the objective of this host range assessment was to identify non-target species that are attacked by $D$. pulchellus and suitable for parasitoid development, then parasitoid age can be said to 
have no effect on host range expression. However, if the objective was to measure the specific rate of non-target attack, thus giving an indication of the non-target's relative rank, then parasitoid age is an important factor, particularly when running choice tests.

The results in Chapters 4 and 5 demonstrate how complex it is to correctly predict how specific factors may affect a parasitoid's oviposition behaviour. This, in turn, makes it difficult to determine which physiological and informational states would most enhance an agent's motivation to oviposit and maximise its host range expression. Hence, Withers and Browne's (2004) guidelines appear not to be appropriate for all entomophagous agents. While they are perhaps a good theoretical starting point, it is clear that more work is needed to understand the endogenous and exogenous factors that affect host range.

The fundamental host range of $D$. pulchellus includes species in Acrolepiidae and Plutellidae, but likely not other families in Yponomeutoidea. This appears to be due to phylogenetic affinity of those non-target hosts with leek moth. The question remaining is whether hosts that are suitable in the laboratory are actually attacked by D. pulchellus under natural conditions. An important knowledge gap here is whether females forage in non-target habitat and, if so, to what extent. Since the suitable non-targets do not occur on Allium host plants, they may be protected by their habitat. If $D$. pulchellus searches exclusively for Allium host plants, then there should be zero non-target risk. If these parasitoids will land on and search any plant releasing dominant sulphur volatiles, then there is a chance of attack on the non-native Plutella spp. Of greatest concern is whether D. pulchellus would forage among vegetation lacking any sulphur-based stimuli. Based on previous research (e.g., Lecomte and Thibout, 1983) this is considered highly improbable and the native non-target, $A$. incertella, on bristly greenbrier is likely outside the ecological host range of $D$. pulchellus. If habitat preference and visitation frequency by $D$. pulchellus must be elucidated before a decision can be made to release this agent in Canada, there are at least two feasible methods to obtain this information. First, wind tunnel assays can effectively reveal odour preference through directional flight (Kitt and Keller, 1998; Yong et al., 2007). In this way, specific odours representing high-risk habitat can be tested directly. The second option is to conduct open field studies in the agent's area of origin and this can be done in one of two ways. Researchers can sample wild populations of non-target species and rear the pupae in containment to observe 
whether D. pulchellus adults emerge (e.g., Haye et al., 1995). Alternatively, non-target hosts shown to be suitable for $D$. pulchellus development (e.g., P. xylostella) can be set up on sentinel host plants in an area where the parasitoids have been released or are known to commonly occur. The latter approach is likely more efficient as it does not require locating naturally occurring, and often elusive, non-target populations.

Several features of $D$. pulchellus make it a very strong candidate for introduction into Canada. In Switzerland, it was one of the two most abundant parasitoid species on leek moth. It was the only parasitoid found attacking the pest in all of the three leek moth generations since it was the sole species obtained in the final generation. Diadromus pulchellus has a quick generation time and although the females do not have a high daily fecundity, they are long-lived. Since the adults are strong fliers, their dispersal capabilities should rival those of the pest. Finally, it is a sister species to Diadromus collaris (Gravenhorst) (Hymenoptera: Ichneumonidae), which has been used extensively in biological control programmes worldwide against Plutella xylostella (Linnaeus) (Lepidoptera: Plutellidae), with proven efficacy (Sarfraz et al., 2005) and no evidence of non-target effects. Based on the findings in this thesis, there are no readily apparent reasons at this point to reject $D$. pulchellus as a classical biological control agent of leek moth. 


\section{References}

Abo-Ghalia, A. and Thibout, E. 1982. Fréquence de la diapause reproductrice en fonction de l'évolution de la photopériode à températures contantes et recherche du stade sensible chez une souche d'Acrolepiopsis assectella [Lep., Yponomeutidae]. Annales de la Société Entomologique de France 18: 173-179.

Allison, J., Jenner, W., Cappuccino, N. and Mason, P.G. 2007. Oviposition and feeding preference of Acrolepiopsis assectella Zell. (Lep., Acrolepiidae). Journal of Applied Entomology 131: 690-697.

Ambriz, S.J., Strand, M.R. and Burkholder, W.E. 1996. Behavioral response of the parasitoid Lariophagus distinguendus (Forst) (Hymenoptera: Pteromalidae) to extracts from cocoons of Lasioderma serricorne Fab. (Coleoptera: Anobiidae) and their effects on subsequent oviposition responses. Biological Control 6: 51-56.

Ankersmitt, G.W. 1953. DDT resistance in Plutella maculipennis (Curt.) (Lepidoptera) in Java. Bulletin of Entomological Research 44: 421-425.

Askew, R.R. and Shaw, M.R. 1986. Parasitoid communities: Their size, structure and development. Pp. 225-264, In: Waage, J. and Greathead, D. (Eds.) Insect Parasitoids. Academic Press, London, UK.

Auerbach, M.J., Connor, E.F. and Mopper, S. 1995. Minor miners and major minors: population dynamics of leaf-mining insects. pp. 83-110, In: Cappuccino, N. and Price, P.W. (Eds.) Population Dynamics: New Approaches and Synthesis. Academic Press, San Diego, USA.

Auger, J., Lecomte, C., Paris, J. and Thibout, E. 1989. Identification of leek moth and diamondback moth frass volatiles that stimulate parasitoid, Diadromus pulchellus. Journal of Chemical Ecology 15: 1391-1398.

Babendreier, D., Bigler, F. and Kuhlmann, U. 2006. Current status and constraints in the assessment of non-target effects. pp.1-14, In: Bigler, F., Babendreier, D. and Kuhlmann, U. (Eds.), Environmental Impact of Invertebrates for Biological Control of Arthropods: Methods and Risk Assessment. CABI Publishing, Wallingford, UK.

Babendreier, D., Kuske, S. and Bigler, F. 2003. Non-target host acceptance and parasitism by Trichogramma brassicae Bezdenko (Hymenoptera: Trichogrammatidae) in the laboratory. Biological Control 26: 128-138.

Bai, B. and Mackauer, M. 1991. Conspecific superparasitism in two parasitoid wasps, Aphidius ervi and Aphelinus asychis: reproductive strategies influence host discrimination. Canadian Entomologist 122: 363-372. 
Bailey, S.D., Bazinet, M.L., Driscoll, J.L. and McCarthy, A.I. 1961. The volatile sulfur compounds of cabbage. Journal of Food Science 26: 163-170.

Barbosa, P. 1998. Conservation Biological Control. Academic Press, San Diego, USA.

Barclay, H.J. 1996. Modelling selection for resistance to methods of insect control in combination. Researches on Population Ecology 38: 75-85.

Barlow, N.D., Barratt, B.I.P., Ferguson, C.M. and Barron, M.C. 2004. Using models to estimate parasitoid impacts on nontarget host abundance. Environmental Entomology 33: 941-948.

Barron, M.C., Barlow, N.D. and Wratten, S.D. 2003. Non-target parasitism of the endemic New Zealand red admiral butterfly (Bassaris gonerilla) by the introduced biological control agent Pteromalus puparum. Biological Control 27: 329-335.

Barratt, B.I.P., Blossey, B. and Hokkanen, H.M.T. 2006. Post-release evaluation of nontarget effects of biological control agents. Pp. 166-186, In: Bigler, F., Babendreier, D. and Kuhlmann, U. (Eds.), Environmental Impact of Invertebrates for Biological Control of Arthropods: Methods and Risk Assessment. CABI Publishing, Wallingford, UK.

Barratt, B.I.P., Evans, A.A., Ferguson, C.M., Barker, G.M., McNeill, M.R. and Phillips, C.B. 1997. Laboratory nontarget host range of the introduced parasitoids Microctonus aethiopoides and $M$. hyperodae (Hymenoptera: Braconidae) compared with field parasitism in New Zealand. Environmental Entomology 26: 694-702.

Barratt, B.I.P., Ferguson, C.M. and McNeill, M.R. 1999. Parasitoid host specificity testing to predict field host range, pp. 70-83, In: Withers, T.M., Barton-Browne, L. and Stanley, J. (Eds.) Host Specificity Testing in Australasia: Towards Improved Assays for Biological Control. CRC for Tropical Pest Management, Brisbane, Australia.

Batra, S.W.T. 1982. Biological control in agroecosystems. Science 215: 134-139.

Bauer, R. 2001. Bemerkungen uber die Ichneumoniden der Alpen mit einigen Neubeschreibungen. Teil III (Hymenoptera, Ichneumonidae, Ichneumoninae). Entomofauna 22: 245-272.

Bekkaoui, A. and Thibout, E. 1992. Rôle de substances cuticulaires non volatiles d'Acrolepiopsis assectella (Lep.: Hyponomeutoidea) dans la reconnaissance de l'hôte par les parasitoïdes Diadromus pulchellus et D. collaris (Hym.: Ichneumonidae). Entomophaga 37: 627-639. 
Bekkaoui, A. and Thibout, E. 1993. Role of the cocoon of Acrolepiopsis assectella (Lep., Hyponomeutidae) in host recognition by the parasitoid Diadromus pulchellus (Hym., Ichneumonidae). Entomophaga 38: 101-113.

Bell, W.J. 1990. Searching behavior patterns in insects. Annual Review of Entomology 35: $447-467$.

Bellows, T.S. and Fisher, T.W. (Eds.) 1999. Handbook of Biological Control: Principles and Applications of Biological Control. Academic Press, San Diego, USA.

Bellows, T.S., Jr, Van Driesche, R.G. and Elkinton, J.S. 1992. Life-table construction and analysis in the evaluation of natural enemies. Annual Review of Entomology 37: 587614.

Bigler, F., Babendreier, D. and Kuhlmann, U. (Eds.) 2006. Environmental Impact of Invertebrates for Biological Control of Arthropods: Methods and Risk Assessment. CABI Publishing, Wallingford, UK.

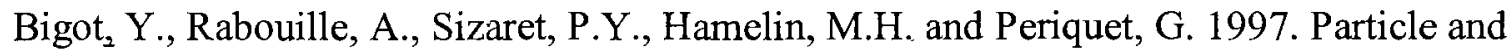
genomic characteristics of a new member of the Ascoviridae: Diadromus pulchellus ascovirus. Journal of General Virology 78: 1139-1147.

Bjorksten, T.A. and Hoffmann, A.A. 1995. Effects of pre-adult and adult experience on host acceptance in choice and non-choice tests in two strains of Trichogramma. Entomologia Experimentalis et Applicata 76: 49-58.

Bjorksten, T.A. and Hoffmann, A.A. 1998. Plant cues influence searching behaviour and parasitism in the egg parasitoid Trichogramma nr. brassicae. Ecological Entomology 23: 355-362.

Briggs, C.J., Murdoch, W.M. and Nisbet, R.M. 1999. Recent developments in theory for biological control of insect pests by parasitoids. pp. 22-42, In: Hawkins, B.A. and Cornell, H.V. (Eds.) Theoretical Approaches to Biological Control. Cambridge University Press, Cambridge, UK.

Brodeur, J. and Vet, L.E.M. 1995. Relationships between parasitoid host-range and hostdefense - a comparative study of egg encapsulation in 2 related parasitoid species. Physiological Entomology 20: 7-12.

Browne, L.B. 1993. Physiologically induced changes in resource-oriented behaviour. Annual Review of Entomology 38: 1-25.

Browne, L.B. and Withers, T.M. 2002. Time-dependent changes in the host-acceptance threshold of insects: implications for host specificity testing of candidate biological control agents. Biocontrol Science and Technology 12: 677-693. 
Bueno, B.H.P., Gutierrez, A.P. and Ruggle, P. 1993. Parasitism by Aphidius ervi (Hym.: Aphidiidae): preference for pea aphid and blue alfalfa aphid (Hom.: Aphididae) and competition with $A$. smithi. Entomophaga 38: 273-284.

Caltagirone, L.E. 1981. Landmark examples in classical biological control. Annual Review of Entomology. 26: 213-232.

Campbell, A., Frazer B.D., Gilbert, N., Gutierrez, A.P. and Mackauer, M. 1974. Temperature requirements of some aphids and their parasites. Journal of Applied Ecology 11: 431-438.

Cappuccino, N. 1991. Mortality of Microrhopala vittata (Coleoptera: Chrysomelidae) in outbreak and nonoutbreak sites. Environmental Entomology 20: 865-871.

Carbone, S. S. and Rivera, A.C. 2003. Egg load and adaptive superparasitism in Anaphes nitens, an egg parasitoid of the Eucalyptus snout-beetle Gonipterus scutellatus. Entomologia Experimentalis et Applicata 106: 127-134.

Carey, J.R. 2001. Insect biodemography. Annual Review of Entomology. 46: 79-110.

Carson, R. 1962. Silent Spring. Hamish Hamilton Ltd., London, UK.

Casagrande, R.A. and Kenis, M. 2004. Evaluation of lily leaf beetle parasitoids for North American introduction. pp. 121-137, In: Van Driesche, R.G. and Reardon, R. (Eds.), Assessing Host Ranges of Parasitoids and Predators Used for Classical Biological Control: A Guide to Best Practice. FHTET, USDA Forest Service, Morgantown, USA.

Causton, C.E. 2004. Predicting the field prey range of an introduced predator, Rodolia cardinalis Mulsant, in the Galapagos. pp. 195-223, In: Van Driesche, R.G. and Reardon, R. (Eds.), Assessing Host Ranges of Parasitoids and Predators Used for Classical Biological Control: A Guide to Best Practice. FHTET, USDA Forest Service, Morgantown, USA.

Cook, R.J. and Baker, K.F. 1983. The Nature and Practice of Biological Control of Plant Pathogens. The American Phytopathological Society, St. Paul, USA.

Coombs, M. 2004. Estimating the host range of the tachinid Trichopoda giacomellii, introduced into Australia for biological control of the green vegetable bug. pp. 143151, In: R.G. Van Driesche and R. Reardon (Eds.) Assessing Host Ranges of Parasitoids and Predators Used for Classical Biological Control: A Guide to Best Practice. FHTET, USDA Forest Service, Morgantown, USA.

Cornell, H.V. and Hawkins, B.A. 1995. Survival patterns and mortality sources of herbivorous insects: some demographic trends. The American Naturalist 145: 563593. 
Cornell, H.V., Hawkins, B.A. and Hochberg, M.E. 1998. Towards an empirically-based theory of herbivore demography. Ecological Entomology 23: 340-349.

Cornell, H.V. and Pimentel, D. 1978. Switching in the parasitoid Nasonia vitripennis and its effects on host competition. Ecology 59: 297-308.

Cramer, H.H., 1967. Plant protection and world crop production. PflanzenschutzNachrichten. Bayer. 20: 1-524.

Danks, H.V. 1978. Modes of seasonal adaptation in the insects. I. Winter survival. Canadian Entomologist 110: 1167-1205.

Daza-Bustamante, P., Fuentes-Contreras, E., L.C. Rodríguez, L.C., Figueroa, C.C. and Niemeyer, H.M. 2002. Behavioural differences between Aphidius ervi populations from two tritrophic systems are due to phenotypic plasticity. Entomologia Experimentalis et Applicata 104: 321-328.

Debach, P. and Bartlett, B.R. 1964. Methods of colonization, recovery and evaluation. pp. 402-426, In: DeBach, P. (Ed.) Biological Control of Insect Pests and Weeds. Chapman and Hall Ltd., London, UK.

Debach, P. and Rosen, D. 1991. Biological Control by Natural Enemies. Cambridge University Press, Cambridge, UK.

Dennill, G.B., Donnelly, D. and Chown, S.L. 1993. Expansion of host-plant range of a biocontrol agent Trichilogaster acaciaelongifoliae (Pteromalidae) released against the weed Acacia longifolia in South Africa. Agriculture, Ecosystems and Environment 43: $1-10$.

Doutt, R.L. 1959. Biology of parasitic Hymenoptera. Annual Review of Entomology 4: 161-182.

Doutt, R.L. and DeBach, P. 1964. Some biological control concepts and questions. pp. 118-142, In: DeBach, P. (Ed.) Biological Control of Insect Pests and Weeds. Chapman and Hall Ltd:, London, UK.

Drost, Y.C., Lewis, W.J., Tumlinson, J.H. 1988. Beneficial arthropod behavior mediated by airborne semiochemicals. V. Influence of Rearing Method, Host Plant, and Adult Experience on Host-Searching Behavior of Microplitis croceipes (Cresson), a Larval Parasitoid of Heliothis. Journal of Chemical Ecology 14: 1607-1616.

Duan, J.J., Ahmad, M., Joshi, K. and Messing. R.H. 1997. Evaluation of the impact of the fruit fly parasitoid Diachasmimorpha longicaudata (Hymenoptera: Braconidae) on a non-target tephritid, Eutreta xanthochaeta (Diptera: Tephritidae). Biological Control 8: $58-64$. 
Duan, J.J. and Messing. R.H. 1996. Response of two Opiine fruit fly parasitoids (Hymenoptera: Braconidae) to the lantana gall fly (Diptera: Tephritidae). Environmental Entomology 25: 1428-1437.

Duan, J.J. and Messing, R.H. 1997. Effect of two Opiine parasitoids (Hymenoptera: Braconidae) introduced for fruit fly control on a native Hawaiian tephritid, Trupanea dubautiae (Diptera: Tephritidae). Biological Control 8: 177-184.

Duan, J.J., Messing, R.H. and Dukas, R. 2000. Host selection of Diachasmimorpha tryoni (Hymenoptera: Braconidae): comparative response to fruit-infesting and gall-forming tephritid flies. Environmental Entomology 29: 838-845.

Dugdale, J.S., Kristensen, N.P., Robinson, G.S. and Scoble, M.J. 1998. The Yponomeutoidea. pp. 119-130, In: Kristensen, N.P. (Ed.) Lepidoptera: Moths and Butterflies. 1. Evolution, Systematics, and Biogeography. Handbook of Zoology, Vol. IV, Part 35. De Gruyter, New York.

Duman, J.G. 2001. Antifreeze and ice nucleator proteins in terrestrial arthropods. Annual Review of Physiology 63: 327-357.

Ehler, L.E. 1990. Environmental impact of introduced biological-control agents: implications for agricultural biotechnology. pp. 85-96, In: Marois, J.J. and Bruening, G. (Eds.) Risk Assessment in Agricultural Biotechnology: Proceedings of the International Conference. Division of Agricultural and Natural Resources, University of California, Oakland, USA.

Eilenberg, J., Hajek, A. and Lomer, C. 2002. Suggestions for unifying the terminology in biological control. BioControl 46: 387-400.

Elkinton, J.S. and Boettner, G.H. 2004. The effects of Compsilura concinnata, an introduced generalist tachinid, on non-target species in North America: a cautionary tale. pp. 4-14, In: Van Driesche, R.G. and Reardon, R. (Eds.) Assessing Host Ranges of Parasitoids and Predators Used for Classical Biological Control: A Guide to Best Practice. FHTET, USDA Forest Service, Morgantown, USA.

Elkinton, J.S., Buonaccorsi, J.P., Bellows Jr., T.S., and Van Driesche, R.G. 1992. Marginal attack rate, k-values and density dependence in the analysis of contemporaneous mortality factors. Researches on Population Ecology 34: 29-44.

Eller, F.J., Tumlinson, J.H. and Lewis, W.J. 1992. Effect of host diet and pre-flight experience on the flight responses of Microplitis croceipes (Cresson). Physiological Entomology 17: 235-240.

Elton, C.S. 1958. The Ecology of Invasions by Animals and Plants. Chapman and Hall, London, UK. 
Environment Canada. 2008. Canadian Climate Normals 1971-2000: Ottawa MacDonaldCartier International Airport. http:/climate.weatheroffice.ec.gc.ca/climate_normals/ results_e.html?Province $=$ ALL\&StationName $=$ Ottawa\&SearchType=Begins $\bar{W}$ ith\&Lo cateBy $=$ Province $\&$ Proximity $=25 \&$ ProximityFrom $=$ City $\&$ StationNumber $=\&$ IDType $=$ MSC \&CityName $=\&$ ParkName $=\&$ LatitudeDegrees $=\&$ LatitudeMinutes $=\&$ Longitude Degrees $=\&$ LongitudeMinutes $=\&$ NormalsClass $=$ A $\&$ SelNormals $=\&$ StnId $=4337 \&$ (accesses 12 June, 2008).

Flanders, S.E. 1947. Elements of host discovery exemplified by parasitic Hymenoptera. Ecology 28: 299-309.

Fletcher, J. P., Hughes, J.P. and Harvey, I.F.. 1994. Life expectancy and egg load affect oviposition decisions of a solitary parasitoid. Proceedings of the Royal Society of London B 258: 163-167.

Floater, G.J. and Zalucki, M.P. 1999. Life tables of the processionary caterpillar Ochrogaster lunifer Herrich-Schäffer (Lepidoptera:Thaumetopoeidae) at local and regional scales. Australian Journal of Entomology 38: 330-339.

Follett, P.A., Duan, J., Messing, R.H. and Jones, V.P. 2000. Parasitoid drift after biological control introductions: re-examining Pandora's Box. American Entomologist 46: $82-94$.

Frank, J.H. 1998. How risky is biological control? Comment. Ecology 79: 1829-1834.

Frediani, D. 1954. Ricerche morfo-biologiche sull'Acrolepia assectella Zell. (Lep. Plutellidae) nell'Italia centrale. Redia 39: 187-249.

Fujiwara, C., Takabayashi, J. and Yano, S. 2000. Oviposition experience on a hostinfested plant affects flight and antennal searching behavior of Cotesia kariyai toward the host-plant complex. Entomologia Experimentalis et Applicata 97: 251-256.

Furlong, M.J., Shi, Z., Liu, S., and Zalucki, M.P. 2004. Evaluation of the impact of natural enemies on Plutella xylostella L. (Lepidoptera: Yponomeutidae) populations on commercial brassica farms. Agricultural and Forest Entomology 6: 311-322.

Gariepy, T.D., Kuhlmann, U., Haye, T., Gillott, C. and Erlandson, M. 2005. A single-step multiplex PCR assay for the detection of European Peristenus parasitoids of Lygus spp. Biocontrol Science and Technology 15: 481-495.

Gauthier, N., Mandon, N., Renault, S. and Bénédet, F. 2004. The Acrolepiopsis assectella silk cocoon: kairomonal function and chemical characterisation. Journal of Insect Physiology 50: 1065-1074. 
Girardoz, S., Tomov, R., Eschen, R., Quicke, D.L.J., and Kenis, M. 2007. Two methods of assessing the mortality factors affecting the larvae and pupae of Cameraria ohridella in the leaves of Aesculus hippocastanum in Switzerland and Bulgaria. Bulletin of Entomological Research 97: 445-453.

Godfray, H.C.J. 1994. Parasitoids: Behavioral and Evolutionary Ecology. 473 pp. Princeton University Press, Princeton, USA.

Goldson, S.L., Mcneill, M.R., Proffitt, J.R. and Barratt, B.I.P. 2005. Host specificity testing and suitability of a European biotype of the braconid parasitoid Microctonus aethiopoides as a biological control agent against Sitona lepidus (Coleoptera: Curculionidae) in New Zealand. Biocontrol Science and Technology 15: 791-813.

Goubault, M., Outreman, Y., Poinsot, D. and Cortesero, A.M. 2005. Patch exploitation strategies of parasitic wasps under intraspecific competition. Behavioral Ecology 16: 693-701.

Gould, F. 1979. Rapid host range evolution in a population of the phytophagous mite Tetranychus urticae (Koch). Evolution 33: 791-802.

Greathead, D.J. 1986. Parasitoids in classical biological control. pp. 290-318 In: Waage, J. and Greathead, D. (Eds.) Insect Parasitoids. Academic Press, London.

Greathead, D.J. 1995. Benefits and risks of classical biological control. pp. 53-63, In: Hokkanen, H.M.T. and Lynch, J.M. (Eds.), Biological Control: Benefits and Risks. Cambridge University Press, Cambridge, UK.

Greathead, D.J. and Greathead, A.H. 1992. Biological control of insect pests by insect parasitoids and predators: the BIOCAT database. Biocontrol News and Information 13: $61 \mathrm{~N}-68 \mathrm{~N}$.

Gurr, G.M., Barlow, N.D., Memmott, J., Wratten, S.D. and Greathead, D.J. 2000. A history of methodological, theoretical and empirical approaches to biological control. pp. 3-37, In: Gurr, G.M and Wratten, S.D. (Eds.) Biological Control: Measures of Success. Kluwer Academic Publishers, Dordrecht, The Netherlands.

Hagvar, E.B. 1991. Ecological problems in the establishment of introduced predators and parasites for biological control. Acta Entomologica Bohemoslovaca 88: 1-11.

Hall, R.W. and Ehler, L.E. 1979. Rate of establishment of natural enemies in classical biological control. Bulletin of the Entomological Society of America 25: 280-282.

Harcourt, D. G. 1969. The development and use of life tables in the study of natural insect populations. Annual Review of Entomology 12: 175-96. 
Hart, A.J., Tullett, A.G., Bale, J.S. and Walters, K.F.A. 2002. Effects of temperature on the establishment potential in the UK of the non-native glasshouse biocontrol agent Macrolophus caliginosus. Physiological Entomology 27: 112-123.

Hatherly, I.S., Bale, J.S., Walters, K.F.A. and Worland, M.R. 2004. Thermal biology of Typhlodromips montdorensis: implications for its introduction as a glasshouse biological control agent in the UK. Entomologia Experimentalis et Applicata 111: 97109 .

Hatherly, I.S., Hart, A.J., Tullett, A.G. and Bale, J.S. 2005. Use of thermal data as a screen for the establishment potential of non-native biological control agents in the UK. BioControl 50: 687-698.

Hawkins, B.A. and Cornell, H.V. (Eds.) 1999. Theoretical Approaches to Biological Control. Cambridge University Press, Cambridge, UK.

Hawkins, B.A., Thomas, M.B, and Hochberg, M.E. 1993. Refuge theory and biological control. Science 262: 1429-1432.

Haye, T., Goulet, H., Mason, P.G. and Kuhlmann, U. 2005. Does fundamental host range match ecological host range? A retrospective case study of a Lygus plant bug parasitoid. Biological Control 35: 55-67.

Henneman, M.L. and Memmott, J. 2001. Infiltration of a Hawaiian community by introduced biological control agents. Science 293: 1314-1316.

Hoffmeister, T.S., Babendreier, D. and Wajnberg, E. 2006. pp. 222-240, In: Bigler, F., Babendreier, D. and Kuhlmann, U. (Eds.) Environmental Impact of Invertebrates for Biological Control of Arthropods: Methods and Risk Assessment. CABI Publishing, Wallingford, UK.

Hokkanen, M.T. and Pimentel, D. 1989. New associations in biological control: theory and practice. Canadian Entomologist. 121: 829-840.

Hopper, K.R. 2001. Research needs concerning non-target impacts of biological control introductions. pp. 39-56. In: Wajnberg, E., Scott, J.K. and Quimby, P.C. (Eds.) Evaluating Indirect Ecological Effects of Biological Control. CABI Publishing, Wallingford, UK.

Hopper, K.R., Roush, R.T. and Powell, W. 1993. Management of genetics of biological control introduction. Annual Review of Entomology 38: 27-51.

Howarth, F.G. 1983. Biological control: panacea or Pandora's box? Proceedings of the Hawaiian Entomological Society 1980 24: 239-244. 
Howarth, F.G. 1991. Environmental impacts of classical biological control. Annual Review of Entomology 36: 485-509.

IPPC. 2005. Code of conduct for the import and release of exotic biological control agents. Publication No. 3, FAO, Rome, Italy. http://www.ippc.int/servlet/BinaryDownloaderSerlet/76047_ISPM_3 pdf?filename=1 146657660135 ISPM3.pdf\&refID $=76047$

Iwasa, Y., Suzuki, Y., and Matsuda, H. 1984. Theory of oviposition strategy in parasitoids. I. Effect of mortality and limited egg number. Theoretical Population Biology 26: 205-227.

Jary, S.G. and Rolfe, S.W. 1945. The leek moth. Agriculture 52: 35-37.

Jenner, W.H. and Kuhlmann, U. 2005. Biological control of leek moth, Acrolepiopsis assectella. Annual Report 2004/2005. 13 pp. Delémont, CABI Europe - Switzerland.

Johnson, M.T., Follett, P.A., Taylor, A.D. and Jones, V.P. 2005. Impacts of biological control and invasive species on a non-target native Hawaiian insect. Oecologia 142: 529-540.

Kaiser, L., Pham-Delegue, M.H. and Masson, C. 1989. Behavioural study of plasticity in host preferences of Trichogramma maidis (Hym.: Trichogrammatidae). Physiological Entomology 14: 53-60.

Kalmes, R. 1984. Examen des problemes posés par la sympatrie sur Acrolepiopsis assectella (Lepidoptère: Hyponomeutoide) dans le sud-est de la France, des deux Hymenoptères Ichneumonides Diadromus pulchellus et Diadromus collaris. 195 pp. PhD thesis, L'Université François Rabelais de Tours, France.

Kalmes, R., Hedderwick, M.-P., Rojas-Rousse, D. and Chessel, D. 1983. Interactions entre deux parasitoïdes mâles dans des chrysalides d'Acrolepiopsis assectella soumis ou non à une compétition intra- et interspécifique. Canadian Journal of Zoology 61: 627-632.

Kalmes, R. and Rojas-Rousse, D. 1988a. Répartition de la ponte et descendance de deux Hyménoptères sympatriques Diadromus collaris et $D$. pulchellus endoparasitoïdes solitaires de la chrysalide d'Acrolepiopsis assectella (Lépidoptère: Plutellidae). Biology of Behaviour 13: 149-163.

Kalmes, R. and Rojas-Rousse, D. 1988b. Dynamique d'un système d'espèces d'Hymenoptères endoparasitoïdes solitaires compétitives: Diadromus collaris et $D$. pulchellus. Acta Oecologia 9: 3-12.

Keane, R.M. and Crawley, M.J. 2002 Exotic plant invasions and the enemy release hypothesis. Trends in Ecology and Evolution 17:164-170. 
Keller, M.A. 1999. Understanding host selection behaviour: the key to more effective host specificity testing. pp. 84-92, In: Withers, T.M., Barton Browne, L. and Stanley, J.N. (Eds), Host Specificity Testing in Australasia: Towards Improved Assays for Biological Control. CRC for Tropical Pest Management, Brisbane, Australia.

Kergeulen, V. and Cardé, R.T. 1996. Increased host acceptance in experienced females of the parasitoid Brachymeria intermedia: which types of oviposition behaviour contribute to experience? Entomologia Experimentalis et Applicata 78: 95-103.

Kidd, N.A.C. and Jervis, M.A. 2005. Population dynamics. pp. 435-523, In: Jervis, M.A. (Ed.) Insects as Natural Enemies: A Practical Perspective. Springer, Dordrecht, The Netherlands.

Kitt, J.T. and Keller, M.A. 1998. Host selection by Aphidius rosae Haliday (Hym., Braconidae) with respect to assessment of host specificity in biological control. Journal of Applied Entomology 122: 57-63.

Knight, A.L. and Norton, G.W. 1989. Economics of agricultural pesticide resistance in arthropods. Annual Review of Entomology 34: 293-313.

Kuhlmann, U., Carl, K.P. and Mills, N.J. 1998. Quantifying the impact of insect predators and parasitoids on populations of the apple ermine moth, Ypnomeuta malinellus (Lepidoptera: Yponomeutidae), in Europe. Bulletin of Entomological Research 88: 165-175.

Kuhlmann, U., Schaffner, U. and Mason, P.G. 2006. Selection of non-target species for host specificity testing. pp. 15-37, In: Bigler, F., Babendreier, D. and Kuhlmann, U. (Eds.) Environmental Impact of Invertebrates for Biological Control of Arthropods: Methods and Risk Assessment. CABI Publishing, Wallingford, UK.

Kyi, A., Zalucki, M.P., and Titmarsh, I.J. 1991. An experimental study of early stage survival of Helicoverpa armigera (Lepidoptera: Noctuidae) on cotton. Bulletin of Entomological Research 81: 263-271.

Labeyrie, V. 1959. Sur la fécondité des femelles vierges de Diadrornus pulchellus Wsm. (Hym : Ichneumonidae). Bulletin de la Société entomologique de France 64: 58-60.

Labeyrie, V. 1960. Contribution a l'étude de la dynamique des populations d'insectes. I. Influence stimulatrice de l'hote Acrolepia assectella $Z$. sur la multiplication d'un hymenoptère Ichneumonidae (Diadromus sp.). 193 pp. PhD Thesis, L'Université de Paris, France.

Labeyrie, V. 1964. Action sélective de la fréquence de l'hôte utilisable (Acrolepia assectella Zel.) sur Diadromus pulchellus Wsm. (Hymenoptera Ichneumonidae): la 
variabilité de la fecondité en fonction de l'intensité de la stimulation. Comptes Rendus Hebdomadaires des Sèances et Mèmoires de la Sociètè de Biologie 259: 3644-3647.

Labeyrie, V. 1966. Sous-famille des Acrolepiinae. pp. 233-249, In : Balachowsky, A.S. (Ed.) Entomologie Appliquée à l'Agriculture. Tome II, Lépidoptères, Vol. 1. Masson et Cie, Paris, France.

Landry, J.-F. 2007. Taxonomic review of the leek moth genus Acrolepiopsis (Lepidoptera: Acrolepiidae) in North America. Canadian Entomologist 139: 319-353.

Larentzaki, E., Powell, G. and Copland, M.J.W. 2007. Effect of temperature on development, overwintering and establishment potential of Franklinothrips vespiformis in the UK. Entomologia Experimentalis et Applicata 124: 143-151.

Leather, S.R., Walters, K.F.A. and Bale, J.S. 1993. The Ecology of Insect Overwintering. Cambridge University Press, Cambridge, UK.

Lecomte, C. and Thibout, E. 1983. Analyse, en olfactomètre tubulaire, de l'influence de différents stimulus olfactifs dans la recherche de l'hôte par Diadromus pulchellus (Hym.: Ichneumonidae). Entomophaga 28: 217-226.

Lecomte, C. and Thibout, E. 1993. Pre- and post-imaginal experience in a specialist parasitoid, Diadromus pulchellus (Hym.: Ichneumonidae). Entomophaga 38: 175184.

Lee, D.A. 1988. Factors affecting mortality of the European corn borer, Ostrinia nubilalis (Hubner), in Alberta. Canadian Entomologist 120: 841-853.

Lee, J.C. and Heimpel, G. 2007. Sugar feeding reduces short-term activity of a parasitoid wasp. Ecological Entomology 32: 99-103.

Lewis, W.J. and Martin, JR., W.R. 1990. Semiochemicals for use with parasitoids: status and future. Journal of Chemical Ecology 16: 3067-3089.

Lewis, W.J., Vet, L.E.M., Tumlinson, J.H., Van Lenteren, J.C. and Papaj, D.R. 1990. Variations in parasitoid foraging behaviour: essential element of a sound biological control theory. Environmental Entomology 19: 1183-1193.

Louda, S.M., Pemberton, R.W., Johnson, M.T. and Follett, P.A. 2003. Nontarget effects the Achilles' Heel of biological control? Retrospective analyses to reduce risk associated with biocontrol introductions. Annual Review of Entomology 48: 365-396.

Luck, R.L., Shepard, B.M. and Kenmore, P.E. 1988. Experimental methods for evaluating arthropod natural enemies. Annual Review of Entomology 33: 367-391. 
Lugowska, B. 1981. Ichneumonidae and Braconidae (Hymenoptera), parasites of Plutella maculipennis Curt. (Lepidoptera, Plutellidae) in Poland. Polskie Pismo Entomologiczne. 51: 355-362.

Lynch, L.D., Hokkanen, H.M.T., Babendreier, D., Bigler, F., Burgio, G., Gao, Z.-H, Kuske, S., Loomans, A., Menzler-Hokkanen, I., Thomas, M.B., Tommasini, G., Waage, J.K., van Lenteren, J.C. and Zeng, Q.-Q. 2001. Insect biological control and non-target effects: a European perspective. pp. 99-125, In: Wajnberg, E., Scott, J.K. and Quimby, P.C. (Eds.) Evaluating Indirect Ecological Effects of Biological Control. CABI Publishing, Wallingford, UK.

Markula, M. 1981. Pests of cultivated plants in Finland in 1980. Annales Agriculturae Fenniae 20: 25-27.

Marohasy, J. 1998. The design and interpretation of host specificity tests for weed biological control with particular reference to insect behaviour. Biocontrol News and Information 19: N13-N20.

Martin, J.L. 1956. The bionomics of the Aspen Blotch Miner, Lithocolletis salicifoliella Cham. (Lepidoptera: Gracillaridae). Canadian Entomologist 88: 155-169.

Mason, P.G., Appleby, M., Callow, K., Allen, J., Fraser, H. and Landry, J.-F. 2006. Leek moth Acrolepiopsis assectella (Lepidoptera: Acrolepiidae) a pest of Allium spp.: biology and minor use insecticide registration. $31 \mathrm{pp}$. Final Project Report to 'Improving Farming Systems Program', AAFC Pest Management Centre (May 15, 2006).

Mattiacci, L., Hütter, E., Schoch, D., Scascighini, N. and Dorn, S. 2000. Plant-odour mediates parasitoid host handling and oviposition in an endophytic tritrophic system. Chemoecology 10: 185-192.

McDonald J.R., Head J., Bale J.S. and Walters K.F.A. 2000. Cold tolerance, overwintering and establishment potential of Thrips palmi. Physiological Entomology 25: 159-166.

McFayden, R.E.C. 1998. Biological control of weeds. Annual Review of Entomology. 43: 369-393.

Messing, R.H. 2001. Centrifugal phylogeny as a basis for non-target testing in biological control: is it relevant for parasitoids? Phytoparasitica 29: 187-189.

Messing, R., Roitberg, B. and Brodeur, J. 2006. Measuring and predicting indirect impacts of biological control: competition, displacement and secondary interactions. pp. 64-77 In: Bigler, F., Babendreier, D. and Kuhlmann, U. (Eds.) Environmental Impact of Invertebrates for Biological Control of Arthropods: Methods and Risk Assessment. CABI Publishing, Wallingford, UK. 
Michaud, J.-P. and Mackauer, M. 1995. Oviposition behaviour of Monoctonus paulensis (Hymenoptera: Aphidiidae): factors influencing reproductive allocation to hosts and host patches. Annals of the Entomological Society of America 88: 220-226.

Minkenberg, O.P.J.M., Tatar, M., and Rosenheim, J.A. 1992. Egg load as a major source of variability in insect foraging and oviposition behavior. Oikos 65: 134-142.

Morris, R.F. 1955. The development of sampling techniques for forest insect defoliators, with particular reference to the spruce budworm. Canadian Journal of Zoology 33: 225-294.

Morris, R.F. 1959. Single-factor analysis in population dynamics. Ecology 40: 580-88.

Murdoch, W.W. and Briggs, C.J. 1996. Theory for biological control: recent developments. Ecology 77: 2001-2013.

Mustata, G. 1992. Role of parasitoid complex in limiting the population of diamondback moth in Moldavia, Romania. pp. 10-14, In: Talekar, N.S. (Ed.), Diamondback moth and other crucifer pests: Proceedings of the second international workshop. Tainan, Taiwan, December 1990. Asian Vegetable Research and Development Center, Taipei.

NAPPO. 2006. Guidelines for Petition for First Release of Exotic Entomophagous Biological Control Agents. RSPM No.12, Secretariat of North American Plant Protection Organization, Ottawa, Canada.

http://www.nappo.org/Standards/REVIEW/RSPMNo.12-rev06-e.pdf

Naranjo, S.E. and Ellsworth, P.C. 2005. Mortality dynamics and population regulation in Bemisia tabaci. Entomologia Experimentalis et Applicata 116: 93-108.

Nechols, J. E., Kauffman, W. C. and Schaefer, P. W. 1992. Significance of host specificity in classical biological control. pp. 41-52 In: Kauffman, W.C. and Nechols, J.E. (Eds.) Selection Criteria and Ecological Consequences of Importing Natural Enemies. Thomas Say Publications in Entomology, Entomological Society of America, Lanham, USA.

Noyes, J.S. 1974. The biology of the leek moth, Acrolepia assectella (Zeller). 254 pp. $\mathrm{PhD}$ Thesis, University of London.

Nyrop, J.P., Shelton, A.M. and Theunissen, J. 1989. Value of a control decision for leek moth infestations in leek. Entomologia Experimentalis et Applicata 53: 167-176.

Obrycki, J.J., Elliott, N.C. and Giles, K.L. 2000. Coccinellid introductions: potential for and evaluation of nontarget effects. pp. 127-145, In: Follett, P.A. and Duan, J.J. (Eds.) Nontarget Effects of Biological Control. Kluwer Academic Publishers, Boston, USA. 
OECD. 2003. Guidance for information requirements for regulations of invertebrates as biological control agents. OECD Environment, Health and Safety Publications. Series on Pesticides 21.

Oerke, E.C., Dehne, H.W., Schönbeck, F. and Weber, A. 1994. Crop Production and Crop Protection: Estimated Losses in Major Food and Cash Crops. Elsevier Science B.V., Amserdam, The Netherlands.

Onstad, D.W. and McManus, M.L. 1996. Risks of host range expansion by parasites of insects. BioScience 46: 430-436.

Papaj, D.R., Rausher, M.D. 1983. Individual variation in host location by phytophagous insects. pp. 77-124, In: Ahmad, S. (Ed.) Herbivorous insects: Host-Seeking Behavior and Mechanisms. Academic Press, New York, USA.

Parra, J.R.P., Vinson, S.B., Gomes, S.M. and Cônsoli, F.L. 1996. Flight response of Habrobracon hebetor (Say) (Hymenoptera : Braconidae) in a wind tunnel to volatiles associated with infestations of Ephestia kuehniella Zeller (Lepidoptera: Pyralidae). Biological Control 6: 143-150.

Pereira, E.J.G., Picanço, M.C., Bacci, L., Crespo, A.L.B., and Guedes, R.N.C. 2007. Seasonal mortality factors of the coffee leafminer, Leucoptera coffeella. Bulletin of Entomological Research 97: 421-432.

Perkins, R.C.L. 1897. The introduction of beneficial insects into the Hawaiian Islands. Nature 55: 499-500.

Petitt, F.L., Turlings, T.C.J. and Wolf, S.P. 1992. Adult experience modifies attraction of the leafminer parasitoid Opius dissitus (Hymenoptera: Braconidae) to volatile semiochemicals. Journal of Insect Behavior 5: 623-634.

Pimentel, D., Glenister, C., Fast, S. and Gallahan, D. 1984. Environmental risks of biological pest controls. Oikos 42: 283-290.

Plaskota, E. and Dabrowski, Z.T., 1986. Biological principles of leek moth (Acrolepiopsis assectella Zeller, Lepidoptera: Plutellidae) control. I. Host plants, damage caused by leek moth larvae, and leek moth parasites. Annals of Warsaw Agricultural University 13: 27-34.

Podoler, H. and Rogers, D. 1975. A new method for the identification of key factors from life-table data. Journal of Animal Ecology 44: 85-114.

Pompanon, F., Fouillet, P. and Bouletreau, M. 1999. Physiological and genetic factors as sources of variation in locomotion and activity rhythm in a parasitoid wasp (Trichogramma brassicae). Physiological Entomology 24: 346-357. 
Price, P.W. 1975. Insect Ecology. John Wiley \& Sons, New York, USA.

Price, P.W. 1980. Evolutionary Biology of Parasites. Princeton University Press, Princeton, USA.

Rasnitsyn, A.P. 1964. Overwintering of ichneumon-flies (Hymenoptera, Ichneumonidae). Entomological Review 43: 24-26.

Rausher, M.D. 1979. Larval habitat suitability and oviposition preference in three related butterflies. Ecology 60: 503-511.

Rogers, M.E. and Potter, D.A. 2004. Biology of Tiphia pygidialis (Hymenoptera: Tiphiidae), a parasitoid of masked chafer (Coleoptera: Scarabaeidae) grubs, with notes on the seasonal occurrence of Tiphia vernalis in Kentucky. Environmental Entomology 33: 520-527.

Roitberg, B.D. 2000. Threats, flies, and video gapes: can evolutionary ecology save biological control? pp. 254-265, In: Hochberg, M.E. and Ives, A.R. (Eds.) Parasitoid Population Biology. Princeton University Press, Princeton, USA.

Roitberg, B. D., Mangel, M., Lalonde, R.G., Roitberg, C.A., van Alphen, J.J.M. and Vet, L.E.M. 1992. Seasonal dynamic shift in patch exploitation by parasitic wasps. Behavioral Ecology 3: 156-165.

Roitberg, B. D., Sircom, J., Roitberg, C.A., van Alphen, J.J.M. and Mangel, M. 1993. Life expectancy and reproduction. Nature 364: 108.

Rojas-Rousse, D. 1980. Examen de quelques problèmes poses à propos des fluctuations $d u$ taux-sexuel chez un hyménoptère entomophage Diadromus pulchellus (Ichneumonidae). 214 pp. PhD Thesis, L'Université François Rabelais de Tours, France.

Salt, R.W. 1963. Delayed inoculative freezing of insects. Canadian Entomologist 95: 1190-1202.

Sands, D. and Liebregts, W. 2005. Biological control of fruit piercing moth (Eudocima fullonia (Clerck)) (Lepidoptera: Noctuidae) in the Pacific: exploration, specificity, and evaluation of parasitoids. pp. 267-276, In: Hoddle (Ed.) Proceedings of the Second International Symposium on Biological Control of Arthropods. Davos, Switzerland, September, 2005. FHTET, USDA Forest Service, Morgantown, USA.

Sands, D. and Van Driesche, R.G. 2000. Evaluating the host range of agents for biological control of arthropods: rationale, methodology and interpretation. pp. 69-83, In: Van Driesche, R.G., Heard, T.A., McClay, A.S. and Reardon, R. (Eds.) Proceedings: Host Specificity Testing of Exotic Arthropod Biological Control Agents: The Biological Basis for Improvement in Safety. Tenth International Symposium on 
Biological Control of Weeds, Bozeman, Montana, July, 1999. FHTET, USDA Forest Service, Morgantown, USA.

Santolamazza-Carbone, S., Pestaña Nieto, M. and Cordero Rivera, A. 2008. Egg resorption behaviour by the solitary egg parasitoid Anaphes nitens under natural conditions. Entomologia Experimentalis et Applicata 127: 191-198.

Sarfraz, M., Keddie, B.A. and Dosdall, L.M. 2005. Biological control of the diamondback moth, Plutella xylostella: a review. Biocontrol Science and Technology 15: $763-789$.

Saunders, D.S. 1965. Larval diapause of maternal origin: induction of diapause in Nasonia vitripennis (Walk.) (Hymenoptera: Pteromalidae). Journal of Experimental Biology 42: 495-508.

Schaffner, J.V., Jr. 1934. Introduced parasites of the brown-tail and gypsy moths reared from native hosts. Annals of the Entomological Society of America 27: 585-592.

Sebald, H., Bauer, R., Schönitzer, K. and Diller, E. 2000. Ichneumonidae, die als Imagines überwintern. (Insecta, Hymenoptera, Ichneumonidae). Entomofauna 21: 285-290.

Secord, D. and Kareiva, P. 1996. Perils and pitfalls in the host specificity paradigm. BioScience 46: 448-453.

Shorthouse, J.D., Zuchlinski, J.A. and Courtin, G.M. 1980. Influence of snow cover on the overwintering of three species of gall-forming Diplolepis (Hymenoptera: Cynipidae). Canadian Entomologist 112: 225-229.

SPSS Inc. (2005) SPSS Base 14.0 User's Guide. Chicago, SPSS Inc.

Stiling, P. 1993. Why do natural enemies fail in classical biological control programs? American Entomologist spring: 31-37

Stoehr, A.M. 2007. Inter- and intra-sexual variation in immune defence in the cabbage white butterfly, Pieris rapae L. (Lepidoptera: Pieridae). Ecological Entomology 32: 188-193.

Strand, M.R. and Obrycki, J.J. 1996. Host specificity of insect parasitoids and predators: many factors influence the host ranges of insect natural enemies. BioScience 46: 422429.

Tauber, C.A., Tauber, M.J. and Masaki, Sinzo. 1986. Seasonal Adaptations of Insects. Oxford University Press, Oxford, UK. 426 pg. 
Thibout, E. 1981. Observations préliminaires et caractérisation de la diapause reproductrice chez la teigne du poireau, Acrolepiopsis assectella Zell, (Lepidoptera, Hyponomeutoidea). Acta Oecologia 2: 171-182.

Thibout, E. 1988. La spécificité de Diadromus pulchellus (Hyménoptère: Ichneumonidae) vis-à-vis de son hôte Acrolepiopsis assectella, la teigne du poireau. Entomophaga 33: 439-452.

Thibout, E. 2005. Role of caterpillar silk thread in location of host pupae by the parasitoid Diadromus pulchellus. Journal of Insect Behavior 18: 817-826.

Thibout, E., Guillot, J.F. and Auger, J. 1993. Microorganisms are involved in the production of volatile kairomones affecting the host seeking behaviour of Diadromus pulchellus, a parasitoid of Acrolepiopsis assectella. Physiological Entomology 18: 176-182.

Thibout, E., Lecomte, C. and Auger, J. 1988. Diadromus pulchellus: search for a host and specificity. pg. 7-14, In: INRA (Ed.), Parasitoid Insects. Lyon, France, September, 1987. Les Colloques de l'INRA, no. 48, Paris, France.

Thibout, E. and Nowbahari, B. 1987. La selection du lieu de nymphose chez la teigne du poireau, Acrolepiopsis assectella (Lep.): déterminisme exogène et variations interpopulations. Annales de la Société Entomologique de France 23 : 183-192.

Thompson, W.R. 1913. Specialization of habit in parasites of insects. Comptes Rendus Hebdomadaires des Sèances et Mèmoires de la Sociètè de Biologie 75: 559-560.

Tiedje, J.M, Colwell, R.K., Grossman, Y.L., Hodson, R.E., Lenski, R.E., Mack, R.N. and Regal, P.J. 1989. The planned introduction of genetically engineered organisms: ecological considerations and recommendations. Ecology 70: 298-315.

Toepfer, S. and Kuhlmann, U. 2006. Constructing life-tables for the invasive maize pest Diabrotica vergifera vergifera (Col.; Chrysomelidae) in Europe. Journal of Applied Entomology 130: 193-205.

Turlings, T.C.J., Tumlinson, J.H., Lewis, W.J. and Vet, L.E.M. 1989. Beneficial arthropod behavior mediated by airborne semiochemicals. VIII. Learning of hostrelated odors induced by a brief contact experience with host by-products in Cotesia marginiventris (Cresson), a generalist larval parasitoid. Journal of Insect Behavior 2: 217-225.

Valemberg, J. and Vago, J.L. 1974. Nomenclature des Ichneumoninae hibernant du Cambresis. Bulletin de la Société Entomologique du Nord de la France. 193:1-4.

Van Driesche, R.G. 2004. Predicting host ranges of parasitoids and predacious insects What are the issues? pp. 1-3 In: R.G. Van Driesche and R. Reardon (Eds.) Assessing 
Host ranges for Parasitoids and Predators Used for Classical Biological Control: a Guide to Best Practice. FHTET, USDA Forest Service, Morgantown, USA.

Van Driesche, R. G. and Bellows, T. S., Jr. 1996. Biological Control. Chapman and Hall, New York, USA.

Van Driesche, R.G. and Hoddle, M.S. 2000. Classical arthropod biological control: measuring success, step by step. pg. 39-75, In: Gurr, G.M and Wratten, S.D. (Eds.) Biological Control: Measures of Success. Kluwer Academic Publishers, Dordrecht, NL.

Van Driesche, R.G. and Murray, T.J. 2004. Overview of testing schemes and designs used to estimate host ranges. pp. 68-89 In: Van Driesche, R.G. and Reardon, R. (Eds.) Assessing Host Ranges of Parasitoids and Predators Used for Classical Biological Control:" A Guide to Best Practice. FHTET, USDA Forest Service, Morgantown, USA.

Van Driesche, R.G. and Reardon, R. (Eds.) 2004. Assessing Host Ranges of Parasitoids and Predators Used for Classical Biological Control: A Guide to Best Practice. FHTET, USDA Forest Service, Morgantown, USA.

van Lenteren, J. C. 1976. The development of host discrimination and the prevention of superparasitism in the parasite Pseudeucoila bochei Weld (Hym.: Cynipidae). Netherlands Journal of Zoology 26: 1-83.

van Lenteren, J.C. 1981. Host discrimination by parasitoids. pp. 153-180 In: Nordlund, D.A., Jones, R.L. and Lewis, W.J. (Eds.) Semiochemicals, Their Role in Pest Control. John Wiley, New York, USA.

Van Lenteren, J.C., Cock, M.J.W., Hoffmeister, T.S. and Sands, D.P.A. 2006. Host specificity in arthropod biological control, methods for testing and interpretation of the data. pp.38-63, In: Bigler, F., Babendreier, D. and Kuhlmann, U. (Eds.) Environmental Impact of Invertebrates for Biological Control of Arthropods: Methods and Risk Assessment. CABI Publishing, Wallingford, UK.

Varley, G.C. and Gradwell, G.R. 1960. Key factors in population studies. Journal of Animal Ecology 29: 399-401.

Velitchkevitch, A.I. 1924. Biological observations on A. assectella, Zell. in the Novgorod government [abstract]. Review of Applied Entomology 12: 356.

Vet, L.E.M. and Dicke, M. 1992. Ecology of infochemical use by natural enemies in a tritrophic context. Annual Review of Entomology 37: 141-172. 
Visser, M.E., van Alphen, J.J. and Nell, H.W. 1990. Adaptive superparasitism and patch time allocation in solitary parasitoids: The influence of the number of parasitoids depleting a patch. Behaviour 114: 21--36.

Vinson, S.B. 1984. Parasitoid-host relationship. pp. 205-233, In: Bell, W.J. and Cardé, R.T. (Eds.) Chemical Ecology of Insects. Sinauer Associates, Sunderland, USA.

Völkl, W. and Mackauer, M. 1990. Age-specific pattern of host discrimination by the aphid parasitoid Ephedrus californicus Baker (Hymenoptera: Aphidiidae). Canadian Entomologist 122: 349-361.

Voukassovitch, P. 1927. Observations biologiques sur les parasites de Plutella maculipennis Curtis. Revue de Pathologie Vegetale et d'Entomologie Agricole de France. 14: 113-118.

Waage, J.K. 1990. Ecological theory and the selection of biological control agents. pp. 135-157, In: Mackauer, M., Ehler, L.E. and Roland, J. (Eds.) Critical Issues in Biological Control. Intercept, Andover, UK.

Wapshere, A.J. 1974. A strategy for evaluating the safety of organisms for biological weed control. Annals of Applied Biology 77: 201-211.

Weisser, W.W. 1994. Age-dependent foraging behaviour and host-instar preference of the aphid parasitoid Lysiphlebus cardui. Entomologia Experimentalis et Applicata 70: $1-10$.

Withers, T.M. and Browne, L.B. 2004. Behavioural and physiological processes affecting outcomes of host range testing. pp. 40-55, In: Van Driesche, R.G. and Reardon, R. (Eds.) Assessing Host Ranges of Parasitoids and Predators Used for Classical Biological Control: A Guide to Best Practice. FHTET, USDA Forest Service, Morgantown, USA.

Yong, T.-H., Pitcher, S., Gardner, J. and Hoffmaun, M.P. 2007. Odor specificity testing in the assessment of efficacy and non-target risk for Trichogramma ostriniae (Hymenoptera: Trichogrammatidae). Biocontrol Science and Technology 17: 135153.

Yu, D.S., van Achterberg, K. and Horstmann, K. 2005. Biological and taxonomical information: Ichneumonoidea 2004. Taxapad Interactive Catalogue, Vancouver, Canada.

Zalucki, M.P., Clarke, A.R., and Malcolm, S.B. 2002. Ecology and behaviour of first instar larval Lepidoptera. Annual Review of Entomology 47: 361-393. 
Zwölfer, H. 1971. The structure and effect of parasite complexes attacking phytophagous host insects. pp. 405-416, In: de Boer, P.J. and Gradwell, G.R. (Eds.) Dynamics of Numbers in Populations. Advanced Study Institute, Oosterbeek, The Netherlands.

Zwölfer, H. and Harris, P. 1971. Host specificity determination of insects for biological control of weeds. Annual Review of Entomology 16: 159-178. 